\title{
Direct Alkynylation of Indole and Pyrrole Heterocycles**
}

\author{
Jonathan P. Brand, Julie Charpentier and Jérôme Waser*
}

Indoles and pyrroles occupy a privileged position in pharmaceuticals, material sciences and natural products. ${ }^{[1]}$ Consequently, methods to synthesize and functionalize these heterocycles are of utmost importance in organic chemistry. ${ }^{[2]}$ Metal-catalyzed cross-coupling is the most often used method for the introduction of (hetero)aryl, vinyl or acetylene groups to indoles and pyrroles, but it requires premodification of the heterocycle. ${ }^{[3]}$ Recently, the direct C-H functionalization of indoles and pyrroles has emerged as a more efficient alternative for the introduction of vinyl and aryl groups. ${ }^{[4]}$ In contrast, direct alkynylation of aromatic compounds are scarce. ${ }^{[5]}$ Recently reported methods include the Ga-catalyzed acetylenation of phenols and anilines, ${ }^{[5 a, 5 b]}$ the Pd-catalyzed alkynylation of $\mathrm{N}$ fused heterocycles, ${ }^{[5 c]}$ anilines ${ }^{[5 d]}$ and indoles, ${ }^{[5]}$ the Ni-catalyzed alkynylation of azoles, ${ }^{[5 f]}$ the reaction of pyrroles with bromoacetylene ketone derivatives ${ }^{[5,5 \mathrm{hh}]}$ and the oxidative $\mathrm{N}$-alkynylation of indoles. ${ }^{[5 i]}$ In the single report on the alkynylation of indoles, ${ }^{[5 \mathrm{e}]}$ the reaction was limited to aryl and alkenyl bromo-acetylenes used in large excess ( 3 equivalents). These substrates cannot be converted into free acetylenes and the large excess of reagent needed limited the practicability of the reaction. Furthermore, the scope of indoles reported was limited, including only methyl, methoxy and ester functional groups. Indoles substituted at position 2 gave low yield, and 3-substituted indoles could not be used. In view of the limited scope reported in the case of indoles and pyrroles, there is an urgent need for new alkynylation methods, especially when considering the importance of acetylenes in organic synthesis. ${ }^{[6]}$ Herein, we report a functional group tolerant gold-catalyzed alkynylation of indole and pyrrole heterocycles. The reaction proceeded in high yield at room temperature under open flask conditions using benziodoxolonederived hypervalent iodine reagent 1d and gives easily deprotected silyl acetylenes products. (Scheme 1).

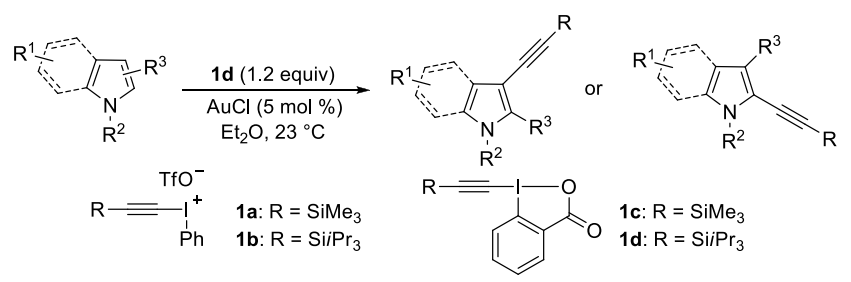

Equation 1.

[*] Jonathan P. Brand, Julie Charpentier and Prof. Dr. J. Waser

Laboratory of Catalysis and Organic Synthesis

Ecole Polytechnique Fédérale de Lausanne

EPFL SB ISIC LCSO, BCH 4306, 1015 Lausanne $(\mathrm{CH})$

Fax: (+) 41216939700

E-mail: jerome.waser@epfl.ch

Homepage: http://isic.epfl.ch/lcso

[**] Dr. Tom Woods (LSYNC) is acknowledged for proofreading this manuscript.

Supporting information for this article is available on the WWW under http://www.angewandte.org or from the author
Due to the limited results obtained with halogen-acetylene derivatives, ${ }^{[5 a-h]}$ we considered using more reactive hypervalent iodine reagents..$^{[7,8]}$ In particular, alkynyliodonium salts as electrophilic/oxidative reagents for acetylene transfer are wellestablished. ${ }^{[\mathrm{a}-\mathrm{g}]}$ Surprisingly, their use for C-H functionalization has not been reported yet, although other hypervalent iodine reagents have been highly successful in arylation and heteroatom transfer reactions. ${ }^{[4 \mathrm{~g}, 4 \mathrm{~h}, 9]}$ However, when reaction conditions reported for the direct arylation of indole $\mathbf{2 a}$ using $\mathrm{Cu}^{[4 \mathrm{~g}]}$ and $\mathrm{Pd}^{[4 \mathrm{~h}]}$ catalysts were examined with alkynyliodonium salts $\mathbf{1 a}$ and $\mathbf{1} \mathbf{b}^{[8 \mathrm{~b}, 8 \mathrm{~d}-\mathrm{f}]}$ and neutral

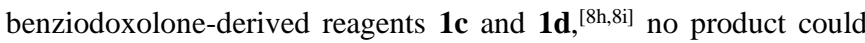
be isolated (Table 1, Entries 1 and 2), as well as with several other metal catalysts. ${ }^{[10]}$ We then turned to Au catalysts. ${ }^{[11]}$ Their capacity to activate $\pi$-multiple bonds ${ }^{[12]}$ is well-established and they have also been used in $\mathrm{C}-\mathrm{C}$ bond formation involving changes of oxidation state at $\mathrm{Au}^{\left[{ }^{[13]}\right.}$ The functionalization of $\mathrm{C}-\mathrm{H}$ bonds using $\mathrm{Au}$ catalysts has been realized in classical hydroarylation reactions. ${ }^{[14]}$ Other reports remained limited to stoichiometric methods ${ }^{[15]}$ or the introduction of heteroatoms. ${ }^{[16]}$ In the case of alkynes, hydroarylation reactions were shown to be favored, and no alkynylation methods based on $\mathrm{Au}$ catalysts could be developed so far. ${ }^{[14 a]}$ The unique combination of $5 \mathrm{~mol} \% \mathrm{AuCl}$ and sterically hindered reagent $\mathbf{1 d}^{[17]}$ in $\mathrm{CH}_{2} \mathrm{Cl}_{2}$ led to the formation of the 3alkynylation product 3a exclusively in $65 \%$ yield (Table 1, Entry 3 ). This constituted the first example of Au-catalyzed C-H alkynylation, as well as an unprecedented use of benziodoxolone-based hypervalent iodine reagents for acetylene transfer.

Table 1. Optimization of alkynylation of indole (2a).

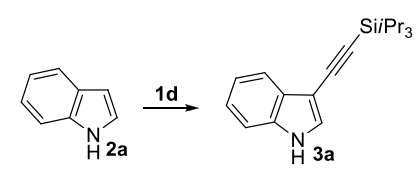

\begin{tabular}{cccc}
\hline Entry & Catalyst & Solvent & Yield $^{[\text {a] }}$ \\
\hline $\mathbf{1}$ & $\mathrm{Pd}(\mathrm{OAc})_{2}$ & $\mathrm{AcOH}$ & $<5 \%$ \\
$\mathbf{2}$ & $\mathrm{Cu}(\mathrm{OTf})_{2}$ & $\mathrm{CH}_{2} \mathrm{Cl}_{2}$ & $<5 \%$ \\
$\mathbf{3}$ & $\mathrm{AuCl}$ & $\mathrm{CH}_{2} \mathrm{Cl}_{2}$ & $65 \%$ \\
$\mathbf{4}$ & $\mathrm{AuCl}_{3}$ & $\mathrm{CH}_{2} \mathrm{Cl}_{2}$ & $56 \%$ \\
$\mathbf{5}$ & $\mathrm{AuNHCCl[b]}$ & $\mathrm{CH}_{2} \mathrm{Cl}_{2}$ & $17 \%$ \\
$\mathbf{6}$ & $\mathrm{AuCl}$ & toluene & $42 \%$
\end{tabular}




\begin{tabular}{cccc}
$\mathbf{7}$ & $\mathrm{AuCl}$ & $\mathrm{Et}_{2} \mathrm{O}$ & $84 \%$ \\
$\mathbf{8}$ & $\mathrm{AuCl}$ & $\mathrm{THF}$ & $85 \%$ \\
$\mathbf{9}$ & $\mathrm{AuCl}$ & $\mathrm{CH}_{3} \mathrm{CN}$ & $82 \%$ \\
$\mathbf{1 0}$ & $\mathrm{AuCl}$ & $\mathrm{DMF}$ & $62 \%$ \\
$\mathbf{1 1}$ & $\mathrm{AuCl}$ & $\mathrm{PrOH}$ & $81 \%$ \\
$\mathbf{1 2}$ & $\mathrm{AuCl}$ & $\mathrm{MeOH}$ & $51 \%$ \\
\hline
\end{tabular}

[a] Reaction conditions: $0.20 \mathrm{mmol} 2 \mathrm{2a}, 5-10 \% \mathrm{~mol}$ catalyst, 1.2 equiv reagent, $4 \mathrm{~mL}$ solvent. Yield was determined via GC-MS. [b] NHC = 1,3-di(2,6-diiso-propylphenyl)-imidazol-2-ylidene.

Examination of several Au catalysts (Table 1, Entries 4 and 5) ${ }^{[18]}$ confirmed that $\mathrm{AuCl}$ was the best catalyst. The reaction worked in a broad range of solvents (Entries 6-12), with the best reproducibility and scope obtained in $\mathrm{Et}_{2} \mathrm{O}$ (Entry 7). Inert conditions or dry solvents were not needed for the reaction and an $86 \%$ yield of $\mathbf{3 a}$ was isolated on a $0.40 \mathrm{mmol}$ scale after column chromatography (Table 2, Entry 1). Importantly, only a slight excess of reagent 1d (20\%) was needed to obtain good yields. This is a distinct advantage of Au catalyst when compared with Pd catalysts, for which extensive dimerization of the acetylene was observed. ${ }^{5 e}$ On a $2.0 \mathrm{mmol}$ scale with only $1 \mathrm{~mol} \%$ of $\mathrm{AuCl}, 84 \%$ of $\mathbf{3 a}$ was isolated, which constitutes the lowest catalyst loading reported so far for $\mathrm{C}-\mathrm{H}$ alkynylation reactions. Furthermore, $63 \%$ of 2 -iodobenzoic acid (4) was recovered via a simple extraction procedure, demonstrating a further advantage of benziodoxolone based reagent. The obtained 2-iodobenzoic acid (4) can then be used for the synthesis of reagent $\mathbf{1 d}$ in two steps and $76 \%$ overall yield with one single recrystallization for purification. The preparation of $\mathbf{1 d}$ is straightforward, and $6 \mathrm{~g}$ of pure 1d has been obtained from 2iodobenzoic acid (4) after a single day of work. Deprotection using TBAF allowed the isolation of the free acetylene indole in $94 \%$ isolated yield.

The scope of the reaction was then examined for several indole heterocycles (Table 2). $N$-Methylindole (2b) gave the desired product in $83 \%$ yield (Entry 2). Both electron-donating (Entries 3-4) and electron-withdrawing (Entries 5-9) groups were tolerated in the reaction, including $\mathrm{OH}$ (Entry 4), $\mathrm{CN}$ (Entry 5), $\mathrm{CO}_{2} \mathrm{H}$ (Entry 6), $\mathrm{NO}_{2}$ (Entry 7), $\mathrm{Br}$ (Entry 8) and I (Entry 9) groups, which have never been reported before. Importantly, yields higher than $90 \%$ were obtained for $\mathrm{Br}$ and I substituents (Entries 8 and 9), making the method orthogonal to classical $\operatorname{Pd}(0)$ cross-coupling chemistry, which is not the case for previously direct alkynylation methods also based on $\operatorname{Pd}(0) .{ }^{[5 c, 5 e]}$ The reaction was also successful for 4,6 and 7 $\mathrm{Br}$ substituted indoles (Entries 10-12). In contrast to previous reports, ${ }^{[5 e]}$ good yields were obtained also in the case of 2substituted indoles (Entries 13-15). Finally, 3-methyl indole, a substrate for which no successful alkynylation has ever been reported, ${ }^{[5 \mathrm{e}]}$ gave the 2-alkynylation product in $76 \%$ yield (Entry 16 ).

We then turned to the alkynylation of pyrroles (Table 3 ). Before our work, there was no report on metal-catalyzed direct alkynylation of these heterocycles. Pyrroles are sensitive compounds that usually require protection of the NH group. ${ }^{[19]}$ In the context of alkynylation reaction, $\mathrm{NH}$ unprotected bromo pyrroles are too unstable to be useful, and the use of classical Sonogashira reactions consequently involves multi-step procedures to give the free pyrrole acetylenes. Gratifyingly, free pyrroles could be used in our protocol (Table 3, Entries 1 and 4-8). For pyrrole itself, the 2-alkynylation product 6 a was obtained in $62 \%$ yield (Entry 1). By using 3 equivalents of pyrrole and one equivalent of $\mathbf{1 d}$, the yield could be increased to $83 \%$. The reaction was sensitive to the steric hindrance on nitrogen: 2-alkynylation product $\mathbf{6 a}$ was obtained exclusively for pyrrole (5a) (Entry 1), a significant amount of 3-alkynylation product $\mathbf{7 b}$ was isolated for $\mathrm{N}$-methyl-pyrrole (5b) (Entry 2) and 3-alkynylation was observed exclusively for $N$-TIPS-pyrrole (5c) (Entry 3). Consequently, the regioselectivity of the reaction can be controlled by the use of easily removable protecting groups. Monosubstituted (Entries 4-6), disubstituted (Entry 7) and trisubstituted (Entry 8) pyrroles could also be used. An electron-withdrawing group was tolerated at the 3-position (Entry 6), but not at the 2-position (result not shown). The use of monosubstituted pyrroles has been rarely reported in metal catalyzed $\mathrm{C}-\mathrm{H}$ functionalization reactions, ${ }^{[4]]}$ and the use of di- and tri- substituted pyrroles is unprecedented.

Table 2. Scope of the Alkynylation Reaction of Indoles.

13


15<smiles>OCc1cc2ccccc2[nH]1</smiles>

16

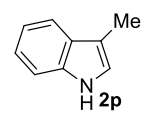

$3 n$

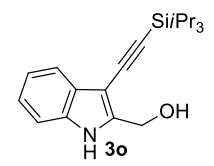

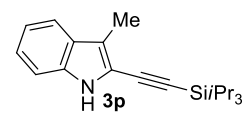

$76 \%$

[a] Reaction conditions: $0.40 \mathrm{mmol} 2,0.48 \mathrm{mmol}$ 1d and $0.02 \mathrm{mmol}$ $\mathrm{AuCl}$ in $8 \mathrm{~mL} \mathrm{Et} 2 \mathrm{O}$ at $23{ }^{\circ} \mathrm{C}$ under air for $12-15 \mathrm{~h}$. Yields are reported for products isolated after column chromatography. [b] $>95 \%$ pure, small amounts of $\mathbf{2}$ could not be separated from the desired product.

Table 3. Scope of the Alkynylation Reaction of Pyrroles.

Entry Substrate

[a] Reaction conditions: $0.40 \mathrm{mmol}$ 5, $0.48 \mathrm{mmol} 1 \mathrm{~d}$ and $0.02 \mathrm{mmol}$ $\mathrm{AuCl}$ in $8 \mathrm{~mL} \mathrm{Et} \mathrm{I}_{2} \mathrm{O}$ at $23^{\circ} \mathrm{C}$ under air for $12-15 \mathrm{~h}$. [b] Yields based on $1 \mathrm{~d}$ with 3 equiv 5 .

Considering the numerous precedents for Au mediated $\pi$ systems activation $^{[12,14]}$ and the rare other examples of $\mathrm{C}-\mathrm{H}$ functionalization, ${ }^{[15,16]}$ at least two hypotheses could be considered for the mechanism: (1) Similar to the $\mathrm{Cu}$ system, ${ }^{[\mathrm{gg}]}$ oxidation of $\mathrm{Au}(\mathrm{I})$ with $1 \mathbf{d}$ to form a $\mathrm{Au}(\mathrm{III})$ acetylene complex I, followed by indole auration and reductive elimination ${ }^{[15 b]}$ or (2) Au-mediated addition of indole to the triple bond of $\mathbf{1 d}$ to form a vinyl $\mathrm{Au}$ complex IIIa or IIIb, ${ }^{[14]}$ followed either by $\beta$-elimination or a $\alpha$ - elimination-1,2-shift sequence ${ }^{[8 b]}$ depending on the regioselectivity of the addition. No Si shift was observed when using $1 \mathbf{d}$ with a ${ }^{13} \mathrm{C}$ label next to $\mathrm{Si}$. Unfortunately, this result does not allow distinguishing between the proposed pathways, as an indole 1,2-shift could also account for this result. Obviously, further experiments are needed to fully understand the reaction mechanism.

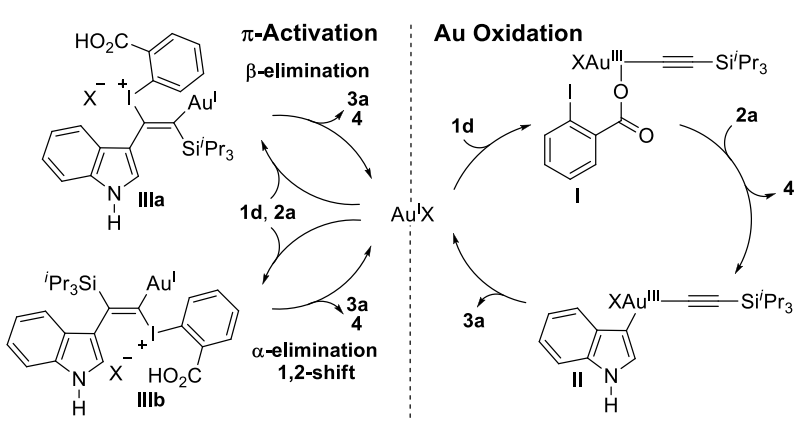

Scheme 1. Working hypothesis for the mechanism of the alkynylation reaction

In conclusion, we have reported the first Au catalyzed direct alkynylation of indole and pyrrole heterocycles using a benziodoxolone-based hypervalent iodine reagent. When compared with the only reported method for the direct alkynylation of indoles, ${ }^{5 e}$ functional group tolerance was greatly increased and unprecedented substitution patterns could be obtained. The reaction efficiency was improved $\left(1 \mathrm{~mol} \%\right.$ catalyst, 1.2 equiv alkyne, $23{ }^{\circ} \mathrm{C}$ compared with $10 \mathrm{~mol} \%$ catalyst, 3 equiv alkyne, $50{ }^{\circ} \mathrm{C}$ ) and easily deprotected silyl acetylenes were obtained. The catalytic, regioselective alkynylation of pyrroles was reported for the first time. The reaction further constitutes a departure from classical Aucatalyzed hydroarylation reactions and was efficient at an unprecedently low catalyst loading for any direct alkynylation methods. The unique properties of benziodoxolone derived hypervalent reagents for acetylene transfer were discovered, which constitutes an important advance in the field of hypervalent iodine chemistry. The exceptional scope of the reaction, as well as the mild reaction conditions and simple experimental procedure (easily accessible reagent, no inert gas, no dry solvent) bode well for the application of the method in organic and medicinal chemistry.

Received: ((will be filled in by the editorial staff)) Published online on ((will be filled in by the editorial staff))

Keywords: Alkynes, C-H Bond Functionalization, Heterocyclic Compd., Hypervalent Iodine, Gold Catalysis.

[1] a) E. C. Taylor, R. A. Jones, Pyrroles. Wiley: New York, 1990; b) The Chemistry of Heterocyclic Compounds. Wiley-Interscience: New York, 1994; Vol. 25; c) R. J. Sundberg, Indoles. Academic: New York, 1996.

[2] a) S. Cacchi, G. Fabrizi, Chem. Rev. 2005, 105, 2873; b) G. R. Humphrey, J. T. Kuethe, Chem. Rev. 2006, 106, 2875.

[3] a) Metal-Catalyzed Cross-Coupling Reactions, Second Edition; ed. A. De Meijere, F. Diederich, Wiley-VCH, 2004; b) M. G. Banwell, T. E. Goodwin, S. Ng, J. A. Smith, D. J. Wong, Eur. J. Org. Chem. 2006, 3043.

[4] a) E. M. Ferreira, B. M. Stoltz, J. Am. Chem. Soc. 2003, 125, 9578; b) B. S. Lane, M. A. Brown, D. Sames, J. Am. Chem. Soc. 2005, 127, 8050 ; c) K. Godula, D. Sames, Science 2006, 312, 67; d) C. Bressy, D. 
Alberico, M. Lautens, J. Am. Chem. Soc. 2005, 127, 13148; e) N. P. Grimster, C. Gauntlett, C. R. A. Godfrey, M. J. Gaunt, Angew. Chem. 2005, 117, 3185; Angew. Chem., Int. Ed. 2005, 44, 3125; f) E. M. Beck, N. P. Grimster, R. Hatley, M. J. Gaunt, J. Am. Chem. Soc. 2006, 128, 2528; g) R. J. Phipps, N. P. Grimster, M. J. Gaunt, J. Am. Chem. Soc. 2008, 130, 8172; h) N. R. Deprez, D. Kalyani, A. Krause, M. S. Sanford, J. Am. Chem. Soc. 2006, 128, 4972; i) D. R. Stuart, K. Fagnou, Science 2007, 316, 1172; j) D. R. Stuart, E. Villemure, K. Fagnou, J. Am. Chem. Soc. 2007, 129, 12072; k) L. C. Campeau, D. J. Schipper; K. Fagnou, J. Am. Chem. Soc. 2008, 130, 3266; 1) N. Lebrasseur, I. Larrosa, J. Am. Chem. Soc. 2008, 130, 2926; m) S. D. Yang, C. L. Sun, Z. Fang, B. H. Li, Y. Z. Li, Z. J. Shi, Angew. Chem. 2008, 120, 1495; Angew. Chem., Int. Ed. 2008, 47, 1473; For reviews, see: n) D. Alberico, M. E. Scott, M. Lautens, Chem. Rev. 2007, 107, 174; o) I. V. Seregin, V. Gevorgyan, Chem. Soc. Rev. 2007, 36, 1173; p) L. Joucla, L. Djakovitch, Adv. Synth. Catal. 2009, 351, 673.

[5] a) K. Kobayashi, M. Arisawa, M. Yamaguchi, J. Am. Chem. Soc. 2002, 124, 8528; b) R. Amemiya, A. Fujii, M. Yamaguchi, Tetrahedron Lett. 2004, 45, 4333; c) I. V. Seregin, V. Ryabova, V. Gevorgyan, J. Am. Chem. Soc. 2007, 129, 7742; d) M. Tobisu, Y. Ano, N. Chatani, Org. Lett. 2009, 11, 3250; e) Y. H. Gu, X. M. Wang, Tetrahedron Lett. 2009, 50, 763; f) N. Matsuyama, K. Hirano, T. Satoh, M. Miura, Org. Lett. 2009, 11, 4156; g) B. A. Trofimov, Z. V. Stepanova, L. N. Sobenina, A. I. Mikhaleva, I. A. Ushakov, Tetrahedron Lett. 2004, 45, 6513; (h) B. A. Trofimov; L. N. Sobenina; Z. V. Stepanova; T. I. Vakulskaya, O. N. Kazheva, G. G. Aleksandrov, O. A. Dyachenko, A. I. Mikhaleva, Tetrahedron 2008, 64, 5541; (i) T. Hamada, X. Ye, S. S. Stahl, J. Am. Chem. Soc. 2008, 130, 833.

[6] F. Diederich, P. J. Stang, R. R. Tykwinski, Acetylene Chemistry: Chemistry, Biology and Material Science; Wiley-VCH, Weinheim, 2005.

[7] a) T.Wirth, Hypervalent iodine chemistry: modern developments in organic synthesis, Vol. 224, Springer, New York, 2003; b) V. V. Zhdankin, P. J. Stang, Chem. Rev. 2008, 108, 5299.

[8] a) F. M. Beringer, S. A. Galton, J. Org. Chem. 1965, 30, 1930. b) M. Ochiai; T. Ito, Y. Takaoka, Y. Masaki, M. Kunishima, S. Tani, Y. Nagao, J. Chem. Soc. Chem. Commun. 1990, 118; c) P. J. Stang, A. M. Arif, C. M. Crittell, Angew. Chem. 1990, 102, 307; Angew. Chem., Int. Ed. Engl. 1990, 29, 287; d) M. D. Bachi, N. Barner, C. M. Crittell, P. J. Stang, B. L. Williamson, J. Org. Chem. 1991, 56, 3912; e) T. Suzuki, Y. Uozumi, M. Shibasaki, J. Chem. Soc. Chem. Commun. 1991, 1593; f) M. D. Bachi, N. Barner, P. J. Stang, B. L. Williamson, J. Org. Chem. 1993, 58, 7923; g) V. V. Zhdankin, P. J. Stang, Tetrahedron 1998, 54, 10927; h) M. Ochiai, Y. Masaki, M. Shiro, J. Org. Chem. 1991, 56, 5511; i) V. V. Zhdankin, C. J. Kuehl, A. P. Krasutsky, J. T. Bolz, A. J. Simonsen, J. Org. Chem. 1996, 61, 6547; Benziodoxolonebased reagents have been neglected so far for atom transfer reactions, with the notable exception of $\mathrm{CF}_{3}$ transfer: j) P. Eisenberger, $\mathrm{S}$. Gischig, A. Togni, Chem. Eur. J. 2006, 12, 2579; k) I. Kieltsch, P. Eisenberger, A. Togni, Angew. Chem. 2007, 119, 768; Angew. Chem.,
Int. Ed. 2007, 46, 754; 1) R. Koller, K. Stanek, D. Stolz, R. Aardoom, K. Niedermann, A. Togni, Angew. Chem. 2009, 121, 4396; Angew. Chem., Int. Ed. 2009, 48, 4332.

[9] a) N. R. Deprez, M. S. Sanford, Inorg. Chem. 2007, 46, 1924; b) K. Eastman, P. S. Baran, Tetrahedron 2009, 65, 3149; (c) R. J. Phipps, M. J. Gaunt, Science 2009, 323, 1593.

[10] No 3-alkynylation product was observed with $\mathrm{Pd}(\mathrm{OAc})_{2}$, $\left[\mathrm{Pd}\left(\mathrm{CH}_{3} \mathrm{CN}\right)_{4}\right]^{2+}\left(\mathrm{BF}_{4}^{-}\right)_{2}, \mathrm{PtCl}_{2}, \mathrm{PtCl}_{4}, \mathrm{Cu}(\mathrm{OTf})_{2}, \mathrm{FeCl}_{3}, \mathrm{ZnCl}_{2}$, $\mathrm{In}(\mathrm{OTf})_{3}, \mathrm{Yb}(\mathrm{OTf})_{3}$ and $\mathrm{HCl}$ or without catalyst. Interestingly, small amounts $(5-10 \%)$ of 2-alkynylation product exclusively were observed with $\mathrm{Pd}$ catalysts in $\mathrm{CH}_{2} \mathrm{Cl}_{2}$.

[11] a) A. S. K. Hashmi, G. J. Hutchings, Angew. Chem. 2006, 118, 8064; Angew. Chem., Int. Ed. 2006, 45, 7896; b) A. S. K. Hashmi, Chem. Rev. 2007, 107, 3180; c) D. J. Gorin, F. D. Toste, Nature 2007, 446, 395.

[12] a) A. Fürstner, P. W. Davies, Angew. Chem. 2007, 119, 3478; Angew. Chem., Int. Ed. 2007, 46, 3410; b) E. Jiménez-Núñez, A. M. Echavarren, Chem. Rev. 2008, 108, 3326; c) S. F. Kirsch, Synthesis 2008, 3183.

[13] a) A. Kar, N. Mangu, H. M. Kaiser, M. Beller, M. K. Tse, Chem. Commun. 2008, 386; b) P. H. Li, L. Wang, M. Wang, F. You, Eur. J. Org. Chem. 2008, 5946; c) H. A. Wegner, S. Ahles, M. Neuburger, Chem. Eur. J. 2008, 14, 11310; d) H. A. Wegner; Chimia 2009, 63, 44; e) G. Z. Zhang, Y. Peng, L. Cui, L. M. Zhang, Angew. Chem. 2009, 121, 3158; Angew. Chem., Int. Ed. 2009, 48, 3112.

[14] a) M. T. Reetz, K. Sommer, Eur. J. Org. Chem. 2003, 3485; b) C. Nevado, A. M. Echavarren, Synthesis 2005, 167; c) C. Ferrer; C. H. M. Amijs, A. M. Echavarren, Chem. Eur. J. 2007, 13, 1358; d) H. C. Shen, Tetrahedron 2008, 64, 3885; e) R. Skouta, C. J. Li, Tetrahedron 2008, 64, 4917.

[15] a) M. S. Kharasch, H. S. Isbell, J. Am. Chem. Soc. 1931, 53, 3053; b) Y. Fuchita, Y. Utsunomiya, M. Yasutake, J. Chem. Soc. Dalton Trans. 2001, 2330.

[16] Z. G. Li, D. A. Capretto, R. O. Rahaman, C. He, J. Am. Chem. Soc. 2007, 129, 12058.

[17] No product was isolated with simple iodoacetylene compounds or reagents 1a-1c. Reagents $\mathbf{1 a}$ and $\mathbf{1 b}$ were obtained in $47 \%$ and $70 \%$ yield respectively from iodosobenzene diacetate, and reagents $\mathbf{1 c}$ and 1d in $55 \%$ and $76 \%$ yield respectively from 2 -iodo benzoic acid (see supporting information for experimental details). Our current work has been focused on silyl protected reagents, as they give easy access to free acetylenes and no direct alkynylation method was available with this class of substrates. Examination of other acetylenebenziodoxolone reagents is currently ongoing in our group and these results will be reported in due course.

[18] Other tested catalysts: $\mathrm{Ph}_{3} \mathrm{PAuCl},\left[\mathrm{Ph}_{3} \mathrm{PAu}\right]^{+} \mathrm{X}^{-}\left(\mathrm{X}=\mathrm{SbF}_{6}, \mathrm{BF}_{4}, \mathrm{OTf}\right)$ $<5 \%$ GC yield.

[19] B. Jolicoeur, E. E. Chapman; A. Thompson, W. D. Lubell, Tetrahedron 2006, 62, 11531. 


\section{Direct Alkynylation}

Jonathan P. Brand, Julie Charpentier and Jérôme Waser *

$$
\text { Page - Page }
$$

Direct Alkynylation of Indole and Pyrrole Heterocycles

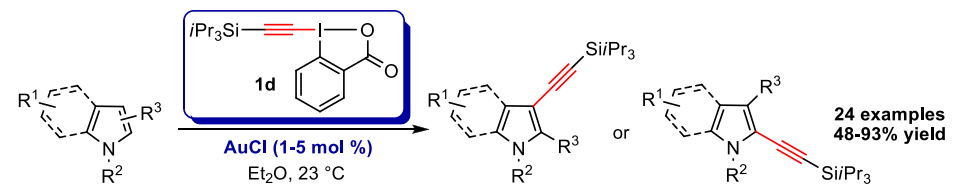

Easy C-H Alkynylation: The first AuCl-catalyzed direct alkynylation of indole and pyrrole heterocycles is described. The unique properties of benziodoxolone alkynyl periodinane $1 \mathbf{d}$ and $\mathrm{Au}$ catalysts allowed the development of a high yielding, operatively simple (room temperature, no dry solvents or inert conditions, commercially available catalyst) reaction for the introduction of silyl acetylenes on a large range of indole and pyrrole heterocycles with broad functional groups and substitution patterns tolerance. 


\section{Supporting information}

\section{Direct Alkynylation of Indole and Pyrrole Heterocycles}

Jonathan P. Brand, Julie Charpentier and Jérôme Waser *

Laboratory of Catalysis and Organic Synthesis, Ecole Polytechnique Fédérale de Lausanne, EPFL SB ISIC LCSO, BCH 4306, 1015 Lausanne, Switzerland

60 pages

1. General Methods S2

2. Preparation of Reagents S2

3. Alkynylation S7

4. Mechanistic Investigation S21

5. Spectra of New compounds S26 


\section{General Methods}

All reactions were carried out in oven dried glassware under an atmosphere of nitrogen, unless stated otherwise. For quantitative flash chromatography technical grade solvents were used. For flash chromatography for analysis, HPLC grade solvents from Sigma-Aldrich were used. THF, $\mathrm{Et}_{2} \mathrm{O}, \mathrm{CH}_{3} \mathrm{CN}_{\text {, }}$ toluene, hexane and $\mathrm{CH}_{2} \mathrm{Cl}_{2}$ were dried by passage over activated alumina under nitrogen atmosphere $\left(\mathrm{H}_{2} \mathrm{O}\right.$ content $<10 \mathrm{ppm}$, Karl-Fischer titration). $\mathrm{NEt}_{3}$ and pyridine were distilled under nitrogen from KOH. Gold chloride was purchased from Aldrich and kept in desiccator under anhydrous condition (decrease of reactivity has been observed for catalyst if prolonged exposition to air ( $c a 1$ month)). All chemicals were purchased from Acros, Aldrich, Fluka, VWR, Aplichem, Maybrige, TCI or Merck and used as such unless stated otherwise. Chromatographic purification was performed as flash chromatography using MachereyNagel silica 40-63, $60 \AA$, using the solvents indicated as eluent with 0.1-0.5 bar pressure.TLC was performed on Merck silica gel $60 \mathrm{~F}_{254}$ TLC glass plates or aluminium plates and visualized with UV light, permanganate stain, CAN stain or Anisaldehyde stain.Melting points were measured on a Büchi B-540 melting point apparatus using open glass capillaries, the data is uncorrected. ${ }^{1} \mathrm{H}-\mathrm{NMR}$ spectra were recorded on a Brucker DPX-400 $400 \mathrm{MHz}$ spectrometer in chloroform-d, DMSO- $\mathrm{d}_{6}$ or $\mathrm{CD}_{3} \mathrm{OD}$, all signals are reported in ppm with the internal chloroform signal at $7.26 \mathrm{ppm}$, the internal DMSO signal at $2.50 \mathrm{ppm}$ or the internal methanol signal at $3.30 \mathrm{ppm}$ as standard. The data is being reported as $(\mathrm{s}=\operatorname{singlet}, \mathrm{d}=$ doublet, $\mathrm{t}=$ triplet, $\mathrm{q}=$ quadruplet, $\mathrm{qi}=$ quintet, $\mathrm{m}=$ multiplet or unresolved, $\mathrm{br}=$ broad signal, app $=$ apparent, coupling constant(s) in $\mathrm{Hz}$, integration, interpretation). ${ }^{13} \mathrm{C}-\mathrm{NMR}$ spectra were recorded with ${ }^{1} \mathrm{H}$ decoupling on a Brucker DPX-400 $100 \mathrm{MHz}$ spectrometer in chloroform-d, DMSO- $\mathrm{d}_{6}$ or $\mathrm{CD}_{3} \mathrm{OD}$, all signals are reported in ppm with the internal chloroform signal at $77.0 \mathrm{ppm}$, the internal DMSO signal at $39.5 \mathrm{ppm}$ or the internal methanol signal at $49.0 \mathrm{ppm}$ as standard. Infrared spectra were recorded on a JASCO FT-IR B4100 spectrophotometer with an ATR PRO410-S and a ZnSe prisma and are reported as $\mathrm{cm}^{-1}(\mathrm{w}=$ weak, $\mathrm{m}=$ medium, $\mathrm{s}=$ strong, $\mathrm{br}=$ broad). Gas chromatographic and low resolution mass spectrometric measurements were performed on a Perkin-Elmer Clarus 600 gas chromatographer and mass spectrometer using a Perkin-Elmer Elite fused silica column (length: $30 \mathrm{~m}$, diameter: $0.32 \mathrm{~mm}$ ) and Helium as carrier gas. High resolution mass spectrometric measurements were performed by the mass spectrometry service of ISIC at the EPFL on a MICROMASS (ESI) Q-TOF Ultima API.

\section{Preparation of Reagents}

\section{Phenyl(trimethylsilylethynyl)iodonium triflate (1a).}

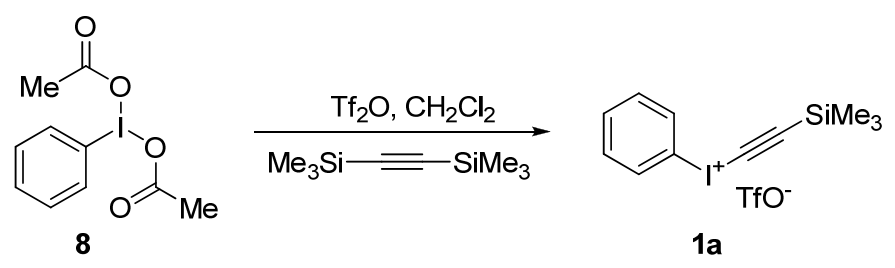

Following a reported procedure, ${ }^{[1]}$ phenyliodonium diacetate (8) $(3.22 \mathrm{~g}, 10.0 \mathrm{mmol}, 1.00$ equiv) was diluted with $\mathrm{CH}_{2} \mathrm{Cl}_{2}(10 \mathrm{~mL})$ and the mixture was stirred for 5 minutes. $\mathrm{Tf}_{2} \mathrm{O}(0.67 \mathrm{~mL}, 5.0 \mathrm{mmol}, 0.50$ equiv.) was added dropwise at $0{ }^{\circ} \mathrm{C}$ and the resulting yellow mixture was stirred $30 \mathrm{~min}$. Bis(trimethylsilyl)acetylene $(2.28 \mathrm{~mL}, 10.0 \mathrm{mmol}, 1.00$ equiv) was added. The mixture was then stirred $2 \mathrm{~h}$ 
and diethyl ether was added to precipitate the product. Filtration afforded 1a $(2.11 \mathrm{~g}, 4.67 \mathrm{mmol}, 47 \%)$ as a colorless solid.

Mp (Dec.) $139-145^{\circ} \mathrm{C}$; Lit.: ${ }^{[1]} 143-146^{\circ} \mathrm{C}$;

${ }^{1} \mathrm{H}$ NMR $\left(400 \mathrm{MHz}, \mathrm{CDCl}_{3}\right) \delta 8.07(\mathrm{~d}, J(H, H)=8.3 \mathrm{~Hz}, 2 \mathrm{H} ; \mathrm{ArH}), 7.66(\mathrm{~s}, 1 \mathrm{H} ; \mathrm{ArH}), 7.55(\mathrm{~m}, 2 \mathrm{H}$; ArH), 0.24 (s, 9 H; TMS);

${ }^{13} \mathrm{C} \mathrm{NMR}\left(100 \mathrm{MHz}, \mathrm{CDCl}_{3}\right) \delta 133.9,132.4,132.2,119.7\left(\mathrm{q},{ }^{1} J(\mathrm{C}, \mathrm{F})=319 \mathrm{~Hz}\right), 119.1,116.2,43.3,-1.1$;

IR v $1448(\mathrm{w}), 1286(\mathrm{~m}), 1253(\mathrm{~m}), 1236(\mathrm{~s}), 1222(\mathrm{~s}), 1161(\mathrm{~m}), 1026(\mathrm{~s}), 988(\mathrm{w}), 863(\mathrm{~m}), 847(\mathrm{~s})$, $742(\mathrm{w}), 714(\mathrm{~m}), 678(\mathrm{w}), 637(\mathrm{~s})$.

Characterization data of 1a corresponded to the literature values. ${ }^{[1]}$

\section{Phenyl(triiso-propylsilyl)iodonium triflate (1b)}

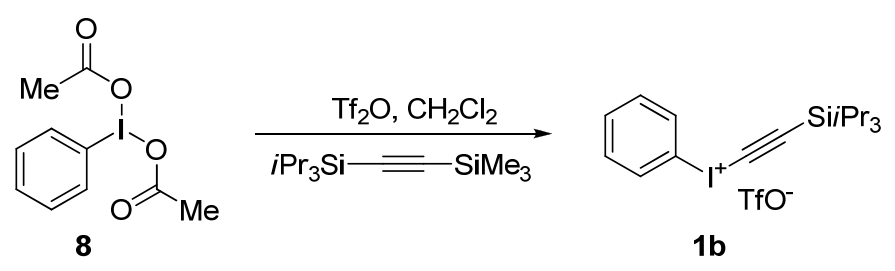

Following a slight modification of the reported procedure, ${ }^{[1]}$ phenyliodonium diacetate (8) (2.53 g, 7.85 mmol, 1.00 equiv) was diluted with $\mathrm{CH}_{2} \mathrm{Cl}_{2}(7 \mathrm{~mL})$ and the mixture was stirred for 5 minutes. $\mathrm{Tf}_{2} \mathrm{O}(0.60$ $\mathrm{mL}, 3.9 \mathrm{mmol}, 0.50$ equiv.) was added dropwise at $0{ }^{\circ} \mathrm{C}$ and the resulting yellow mixture was stirred $30 \mathrm{~min}$. (Trimethylsilyl)(triiso-propylsilyl)acetylene $(2.00 \mathrm{~g}, 7.86 \mathrm{mmol}, 1.00$ equiv) was added and the mixture was then stirred $2 \mathrm{~h}$. Water was then added $(30 \mathrm{~mL})$ followed by extraction of the aqueous layer with $\mathrm{CH}_{2} \mathrm{Cl}_{2}(2 \mathrm{x}$ $30 \mathrm{~mL}$ ). The combined organic layers were dried over $\mathrm{MgSO}_{4}$, filtered and the solvent was evaporated under reduced pressure. The resulting solid was triturated in hexane $(10 \mathrm{~mL})$. Filtration and removal of solvent in vacuo afforded phenyl(triiso-propylsilyl)iodonium triflate (1b) $(2.90 \mathrm{~g}, 11.2 \mathrm{mmol}, 70 \%)$ as a colorless solid.

Mp $109-114^{\circ} \mathrm{C}$;

${ }^{1} \mathrm{H}$ NMR (400 MHz, $\left.\mathrm{CDCl}_{3}\right) \delta 8.09$ (m, $\left.2 \mathrm{H} ; \mathrm{ArH}\right), 7.65$ (m, $\left.1 \mathrm{H} ; \mathrm{ArH}\right), 7.52$ (m, $\left.2 \mathrm{H} ; \mathrm{ArH}\right), 1.15-1.01$ (m, $21 \mathrm{H}$; TIPS);

${ }^{13} \mathrm{C}$ NMR $\left(100 \mathrm{MHz}, \mathrm{CDCl}_{3}\right) \delta 133.7,132.5,132.4,120.3\left(\mathrm{q},{ }^{1} J(\mathrm{C}, \mathrm{F})=319 \mathrm{~Hz}\right), 117.6,117.6,44.9$, $18.3,11.1$

IR v $3288(\mathrm{w}), 3088(\mathrm{~m}), 2949(\mathrm{~m}), 2894(\mathrm{~m}), 2869(\mathrm{w}), 1563(\mathrm{~m}), 1467(\mathrm{w}), 1451(\mathrm{w}), 1388(\mathrm{w}), 1281$ (s), $1236(\mathrm{~s}), 1221$ (s), $1174(\mathrm{~s}), 1068(\mathrm{w}), 1028(\mathrm{~s}), 988(\mathrm{~m}), 916(\mathrm{~m}), 884(\mathrm{~m}), 736$ (s), $679(\mathrm{~m}), 639$ (s);

HRMS (ESI) calcd for $\mathrm{C}_{17} \mathrm{H}_{26} \mathrm{ISi}^{+}$(M-OTf) 385.0843; found 385.0812.

1-Hydroxy-1,2-benziodoxol-3(1H)-one (9)

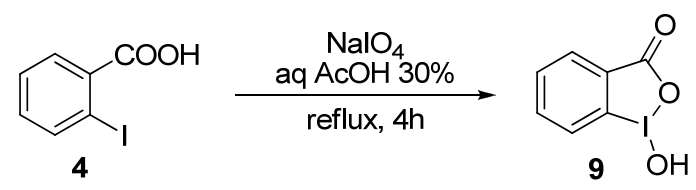


Following a reported procedure, ${ }^{[2]} \mathrm{NaIO}_{4}(6.7 \mathrm{~g}, 31 \mathrm{mmol} ; 1.0$ equiv) and 2-iodobenzoic acid (4) (7.4 g, $30 \mathrm{mmol}, 1.0$ equiv) were suspended in $30 \%$ (v:v) aq. AcOH $(45 \mathrm{~mL})$. The mixture was vigorously stirred and refluxed for $4 \mathrm{~h}$. The reaction mixture was then diluted with cold water $(120 \mathrm{~mL})$ and allowed to cool to room temperature, protecting it from light. After $1 \mathrm{~h}$, the crude product was collected by filtration, washed on the filter with ice water $(3 \times 30 \mathrm{~mL})$ and acetone $(3 \times 30 \mathrm{~mL})$, and air-dried in the dark to give the pure product 9 (7.3 g, $19 \mathrm{mmol}, 92 \%)$ as a colorless solid.

${ }^{1} \mathrm{H}$ NMR $\left(400 \mathrm{MHz},\left(\mathrm{CD}_{3}\right)_{2} \mathrm{SO}\right) \delta 8.02(\mathrm{dd}, J(H, H)=1.4,7.7, \mathrm{~Hz}, 1 \mathrm{H} ; \mathrm{Ar} H), 7.97(\mathrm{~m}, 1 \mathrm{H} ; \mathrm{Ar} H), 7.85$ $(\mathrm{dd}, J(H, H)=0.7,8.2, \mathrm{~Hz}, 1 \mathrm{H} ; \mathrm{Ar} H), 7.71(\mathrm{td}, J(H, H)=1.2,7.6 \mathrm{~Hz}, 1 \mathrm{H} ; \mathrm{Ar} H)$;

${ }^{13} \mathrm{C}$ NMR $\left(100 \mathrm{MHz},\left(\mathrm{CD}_{3}\right)_{2} \mathrm{SO}\right) \delta 167.7,134.5,131.5,131.1,130.4,126.3,120.4 ;$

IR v $3083(\mathrm{w}), 3060(\mathrm{w}), 2867(\mathrm{w}), 2402(\mathrm{w}), 1601(\mathrm{~m}), 1585(\mathrm{~m}), 1564(\mathrm{~m}), 1440(\mathrm{~m}), 1338(\mathrm{~s}), 1302$ (m), 1148 (m), 1018 (w), 834 (m), 798 (w), 740 (s), 694 (s), 674 (m), 649 (m);

The characterization data for compounds 9 corresponded to the reported values. ${ }^{[2]}$

\section{1-[(Trimethylsilyl)ethynyl]-1,2-benziodoxol-3(1H)-one (1c)}

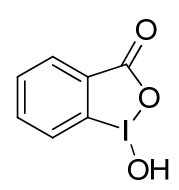

9

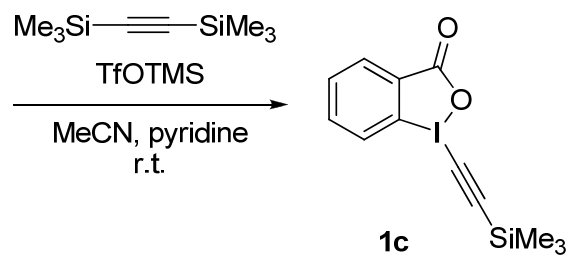

Following a reported procedure, ${ }^{[3]}$ trimethylsiyltriflate $(2.8 \mathrm{~mL}, 15 \mathrm{mmol}, 1.4$ equiv, freshly distilled $)$ was added dropwise to a stirred solution of 2-iodosylbenzoic acid (9) (3.00 g, $11.4 \mathrm{mmol}, 1.00 \mathrm{equiv})$ in acetonitrile $(85 \mathrm{~mL})$ until the mixture turned colorless. Bis(trimethylsilyl)acetylene $(2.14 \mathrm{~g}, 12.5 \mathrm{mmol}, 1.10$ equiv) was then added dropwise, followed, after $20 \mathrm{~min}$, by the addition of pyridine $(1.2 \mathrm{~mL}, 15 \mathrm{mmol}, 1.4$ equiv). The mixture was stirred $30 \mathrm{~min}$. The solvent was then removed under reduced pressure and the yellow crude oil was dissolved in dichloromethane $(80 \mathrm{~mL})$. The organic layer was washed with a large amount of water $(130 \mathrm{~mL})$, and the aqueous layer was extracted with $\mathrm{CH}_{2} \mathrm{Cl}_{2}(3 \times 65 \mathrm{~mL})$. The organic layer was washed with brine $(130 \mathrm{~mL})$, dried over $\mathrm{MgSO}_{4}$, filtered and the solvent was evaporated under reduced pressure. Recrystallization from acetonitrile $(2.3 \mathrm{~mL})$ afforded $1 \mathrm{c}(2.35 \mathrm{~g}, 6.84 \mathrm{mmol}, 60 \%$ yield $)$ as a colorless solid.

Mp: $143-145^{\circ} \mathrm{C}(\mathrm{dec})$;

${ }^{1} \mathrm{H}$ NMR $\left(400 \mathrm{MHz}, \mathrm{CDCl}_{3}\right) \delta 8.42(\mathrm{dd}, J(H, H)=1.9,6.4 \mathrm{~Hz}, 1 \mathrm{H} ; \mathrm{Ar} H), 8.19(\mathrm{~m}, 1 \mathrm{H} ; \mathrm{Ar} H), 7.78(\mathrm{~m}, 2$ $\mathrm{H} ; \mathrm{ArH}), 0.32$ (s, $9 \mathrm{H}$; TMS);

${ }^{13} \mathrm{C}$ NMR (100 MHz, $\left.\mathrm{CDCl}_{3}\right)$ 166.4, 134.9, 132.6, 131.7, 131.4, 126.1, 117.2, 115.4, 64.2, -0.5;

IR $v 3389(\mathrm{w}), 2967(\mathrm{w}), 1617(\mathrm{~s}), 1609(\mathrm{~s}), 1562(\mathrm{~m}), 1440(\mathrm{w}), 1350(\mathrm{~m}), 1304(\mathrm{w}), 1254(\mathrm{w}), 1246$ (w), $1112(\mathrm{w}), 1008(\mathrm{w}), 852(\mathrm{~s}), 746(\mathrm{~m}), 698(\mathrm{~m}), 639(\mathrm{~m})$;

The characterization data for compounds 1c corresponded to the reported values. ${ }^{[3]}$

[2] L. Kraszkiewicz, L. Skulski, Arkivoc, 2003, 6, 120.

[3] V. V.Zhdankin, C. J Kuehl, A. P Krasutsky, J. T. Bolz, A. J. Simonsen, J. Org. Chem. 1996, 61, 6547. 


\section{Triiso-propylsilyl trimethylsilylacetylene (11)}

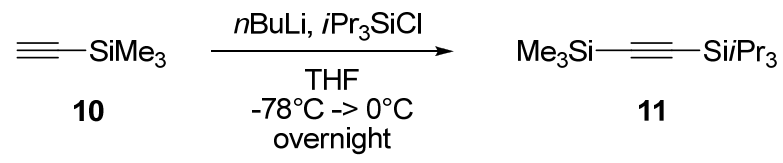

Following a reported procedure, ${ }^{[4]} n$-butyllithium $(2.5 \mathrm{M}$ in hexanes, $12.0 \mathrm{~mL}, 29.9 \mathrm{mmol}, 0.98$ equiv) was added dropwise to a stirred solution of ethynyltrimethylsilane (10) $(3.0 \mathrm{~g}, 30 \mathrm{mmol}, 1.0$ equiv) in THF $\left(48 \mathrm{~mL}\right.$ ) at $-78^{\circ} \mathrm{C}$. The mixture was then warmed to $0{ }^{\circ} \mathrm{C}$ and stirred for $5 \mathrm{~min}$. The mixture was then cooled back to $-78{ }^{\circ} \mathrm{C}$ and chlorotriiso-propylsilane $(6.4 \mathrm{~mL}, 30 \mathrm{mmol}, 1.0$ equiv) was added dropwise. The mixture was then allowed to warm to room temperature and stirred overnight. A saturated solution of ammonium chloride $(40 \mathrm{~mL})$ was added, and the reaction mixture was extracted with diethyl ether $(2 \times 60$ $\mathrm{mL}$ ). The organic layer was washed with water and brine, then dried over $\mathrm{MgSO}_{4}$, filtered and concentrated under reduced pressure to obtain a colorless liquid which was further purified by Kugelrohr distillation (56$\left.57^{\circ} \mathrm{C} / 0.25 \mathrm{mmHg}\right)$ to yield 11 (7.16 g, $28.0 \mathrm{mmol}, 92 \%$ yield) as a colorless liquid.

${ }^{1} \mathrm{H}$ NMR (400 MHz, $\left.\mathrm{CDCl}_{3}\right) \delta 1.08$ (m, $21 \mathrm{H}$; TIPS), 0.18 (s, $9 \mathrm{H}$; TMS);

IR v $2959(\mathrm{~m}), 2944(\mathrm{~m}), 2896(\mathrm{w}), 2867(\mathrm{~m}), 1464(\mathrm{w}), 1385(\mathrm{w}), 1250(\mathrm{~m}), 996(\mathrm{w}), 842(\mathrm{~s}), 764(\mathrm{~s})$, $675(\mathrm{~m}), 660(\mathrm{~m})$.

Characterization data of $\mathbf{1 1}$ corresponded to the literature values. ${ }^{[4]}$

\section{1-[(Triiso-propylsilyl)ethynyl]-1,2-benziodoxol-3(1H)-one (1d)}

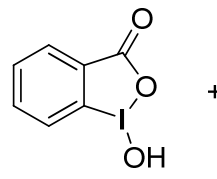

9

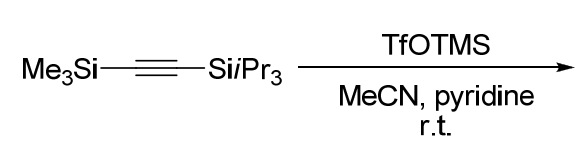

11

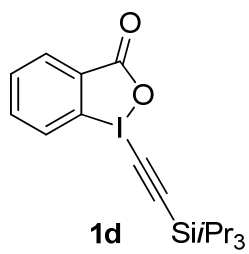

Following a reported procedure, ${ }^{[3]}$ trimethylsilyltriflate $(3.6 \mathrm{~mL}, 20 \mathrm{mmol}, 1.1$ equiv, freshly distilled) was added dropwise to a stirred solution of 2-iodosylbenzoic acid (9) $(4.7 \mathrm{~g}, 18 \mathrm{mmol}, 1.0$ equiv) in acetonitrile (140 mL). (Trimethylsilyl)(triiso-propylsilyl)acetylene (11) (5.0 g, $20 \mathrm{mmol}, 1.1$ equiv) was then added dropwise, followed, after $15 \mathrm{~min}$, by the addition of pyridine (1.5 mL, $20 \mathrm{mmol}$, 1.1 equiv). The mixture was stirred $10 \mathrm{~min}$. The solvent was then removed under reduced pressure and the yellow crude oil was dissolved in dichloromethane $(50 \mathrm{~mL})$. The organic layer was washed with $\mathrm{HCl} 1 \mathrm{M}(50 \mathrm{~mL})$ and the aqueous layer was extracted with $\mathrm{CH}_{2} \mathrm{Cl}_{2}(50 \mathrm{~mL})$. The organic layers were combined, washed with a saturated solution of $\mathrm{NaHCO}_{3}(2 * 50 \mathrm{~mL})$, dried over $\mathrm{MgSO}_{4}$, filtered and the solvent was evaporated under reduced pressure. Recrystallization from acetonitrile $(c a 35 \mathrm{~mL})$ afforded $\mathbf{1 d}(6.3 \mathrm{~g}, 15 \mathrm{mmol}, 83 \%)$ as a colorless solid.

$\mathrm{Mp}$ (Dec.) $170-176^{\circ} \mathrm{C}$;

${ }^{1} \mathrm{H}$ NMR $\left(400 \mathrm{MHz}, \mathrm{CDCl}_{3}\right) \delta 8.44(\mathrm{~m}, 1 \mathrm{H} ; \mathrm{ArH}), 8.29(\mathrm{~m}, 1 \mathrm{H} ; \mathrm{ArH}), 7.77$ (m, $\left.2 \mathrm{H} ; \mathrm{ArH}\right), 1.16(\mathrm{~m}, 21$ H; TIPS);

${ }^{13} \mathrm{C} \mathrm{NMR}\left(100 \mathrm{MHz}, \mathrm{CDCl}_{3}\right) \delta 166.4,134.6,132.3,131.4,131.4,126.1,115.6,114.1,64.6,18.4,11.1$;

[4] C J. Helal, P. A. Magriotis, E. J. Corey, J. Am. Chem. Soc. 1996, 118, 10938. 
IR v $2943(\mathrm{~m}), 2865(\mathrm{~m}), 1716(\mathrm{~m}), 1618(\mathrm{~m}), 1604(\mathrm{~s}), 1584(\mathrm{~m}), 1557(\mathrm{~m}), 1465(\mathrm{~m}), 1439(\mathrm{w}), 1349$ (m), 1291 (m), 1270 (w), 1244 (m), 1140 (m), 1016 (m), 999 (m), 883 (m), 833 (m), 742 (m), 702 (s), 636 (m).

Characterization data of $\mathbf{1 d}$ corresponded to the literature values. ${ }^{[3]}$

\section{2-Iodo-1-triiso-propylsilyl acetylene (13)}

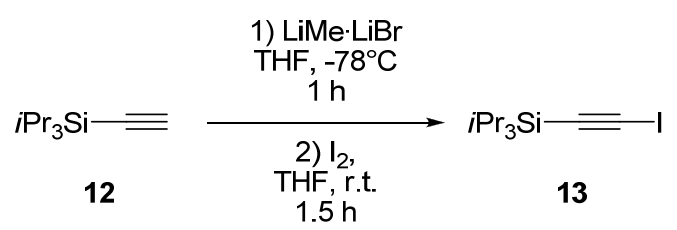

Following a reported procedure, ${ }^{[5]} \mathrm{MeLi} \cdot \operatorname{LiBr}(1.5 \mathrm{M}$ in diethyl ether, 1.1.mL, $1.6 \mathrm{mmol}, 1.0$ equiv) was added to a stirred solution of triiso-propylsilylacetylene $(\mathbf{1 2})(0.36 \mathrm{~mL}, 1.6 \mathrm{mmol}, 1.0$ equiv) in dry THF (1.8 $\mathrm{mL}$ ), cooled at $-78{ }^{\circ} \mathrm{C}$, and the mixture was allowed to react for $1 \mathrm{~h}$ at that temperature. A solution of $\mathrm{I}_{2}$ (457 $\mathrm{mg}, 1.80 \mathrm{mmol}, 1.25$ equiv) in dry THF $(2.7 \mathrm{~mL})$ was then added dropwise and the mixture was stirred for $1.5 \mathrm{~h}$ at $-78^{\circ} \mathrm{C}$. The mixture was then diluted with brine $(6 \mathrm{~mL})$ and the aqueous layer was extracted with ether $(3 \times 10 \mathrm{~mL})$. The combined organic layers were washed with a saturated aqueous solution of $\mathrm{Na}_{2} \mathrm{~S}_{2} \mathrm{O}_{3}$ (3 x $20 \mathrm{~mL}$ ), dried over $\mathrm{MgSO}_{4}$ and concentrated under reduced pressure. Purification by column chromatography (hexane) afforded 2-iodo-1-triiso-propylsilyl acetylene (13) (470 mg, $1.52 \mathrm{mmol}, 94 \%)$ as a colorless oil.

${ }^{1} \mathrm{H}$ NMR (400 MHz, $\left.\mathrm{CDCl}_{3}\right) \delta 1.10-1.04$ (m, $21 \mathrm{H}$; TIPS);

${ }^{13} \mathrm{C} \mathrm{NMR}\left(100 \mathrm{MHz}, \mathrm{CDCl}_{3}\right) \delta 100.8,18.5,11.4$ (one acetylene carbon was not resolved).

Characterization data of $\mathbf{1 3}$ corresponded to the literature values. ${ }^{[5]}$

\section{Substrate synthesis:}

\section{2-Phenyl-1H-pyrrole}

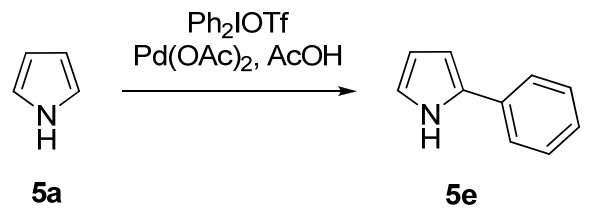

Following a slightly modified procedure, ${ }^{[6]}$ pyrrole $(2.1 \mathrm{~mL}, 30 \mathrm{mmol}, 12$ equiv) was added to a stirring solution of $\mathrm{Pd}(\mathrm{OAc})_{2}(34 \mathrm{mg}, 0.15 \mathrm{mmol}, 0.05$ equiv) in $\mathrm{AcOH}(30 \mathrm{~mL})$ under air. After 5 min stirring, $\mathrm{Ph}_{2} \mathrm{IOTf}$ ( $1.1 \mathrm{~g}, 2.6 \mathrm{mmol}, 1.0$ equiv) was added. The reaction was stirred at room temperature for $15 \mathrm{~h}$ and then filtrated over celite. After concentration under reduced pressure, the mixture was dissolved in DCM (50 $\mathrm{mL})$ and washed twice with a saturated solution of $\mathrm{NaHCO}_{3}(2 \times 30 \mathrm{~mL})$. The organic layer was dried over $\mathrm{MgSO}_{4}$ and concentrated under vacuum. Purification by column chromatography (PET/EtOAc 95/5, Rf=0.2) afforded 2-Phenyl- $1 H$-pyrrole 5e (171 mg, $1.07 \mathrm{mmol}, 41 \%$ yield) as a colorless oil. Purity $\geq 90 \%$.

${ }^{1} \mathrm{H}$ NMR $\left(\mathrm{CDCl}_{3}, 400 \mathrm{MHz}\right) \delta 8.46$ (br s, $\left.1 \mathrm{H} ; \mathrm{NH}\right), 7.51(\mathrm{dm}, J(H, H)=7.2 \mathrm{~Hz}, 2 \mathrm{H} ; \mathrm{PhH}), 7.39$ (m, $2 \mathrm{H}$; $\mathrm{PhH}), 7.24$ (m, $1 \mathrm{H} ; \mathrm{PhH}), 6.90$ (m, $1 \mathrm{H}$; pyrrole H), 6.56 (m, $1 \mathrm{H}$; pyrrole H), 6.33 (m, $1 \mathrm{H}$; pyrrole H);

[5] S. López, F.Fernández-Trillo, P. Midón, L. Castedo, L. Saá, J. Org. Chem., 2005, 70, 6346.

[6] N. R. Deprez, D. Kalyani, D. Krause, M. S. Sanford, J. Am. Chem. Soc. 2006, 128, 4972. 
${ }^{13} \mathrm{C} \mathrm{NMR}\left(\mathrm{CDCl}_{3}, 100 \mathrm{MHz}\right) \delta: 132.8,132.1,128.9,126.2,123.9,118.8,110.2,106.0$.

Characterization data of $5 \mathbf{e}$ corresponded to the literature values. ${ }^{[6]}$

\section{Alkynylation Reaction}

\section{Optimization procedure:}

1d (103 mg, $0.240 \mathrm{mmol}, 1.2$ equiv) was added to a stirring solution of catalyst ( $0.010 \mathrm{mmol}, 0.05$ equiv) and indole (2a) (23 mg, $0.20 \mathrm{mmol}, 1.0$ equiv) in the indicated solvent (4 mL) under air (see Table 1). The reaction was sealed and stirred at room temperature for 12-15 hrs. The reaction mixture was then filtered over silica gel and the filtrate was concentrated in vacuo. The residue was diluted with dichloromethane (1 $\mathrm{mL}$, solution A). $0.1 \mathrm{~mL}$ of a solution of decane $0.02 \mathrm{M}$ in dichloromethane and $0.8 \mathrm{~mL}$ of dichloromethane were added to $0.1 \mathrm{~mL}$ of solution $\mathbf{A}$. The resulting solution was injected into GC-MS and the following oven program was followed: Initial temperature: $50^{\circ} \mathrm{C}$, Ramp: $10.0^{\circ} \mathrm{C} / \mathrm{min}$ to $250{ }^{\circ} \mathrm{C}$, hold 25 min at $250{ }^{\circ} \mathrm{C}$.

\section{General procedure (Table 2 and 3):}

1d (206 mg, $0.480 \mathrm{mmol}, 1.2$ equiv) was added to a stirring solution of $\mathrm{AuCl}$ (4.6 mg, $0.020 \mathrm{mmol}, 0.05$ equiv) and the corresponding indole/pyrrole $\left(0.400 \mathrm{mmol}, 1.0\right.$ equiv) in $\mathrm{Et}_{2} \mathrm{O}(8 \mathrm{~mL})$ under air. The reaction was sealed and stirred at room temperature for $12-15 \mathrm{hrs}$. $\mathrm{Et}_{2} \mathrm{O}(10 \mathrm{~mL})$ was added, the organic layer was washed twice with $\mathrm{NaOH} 0.1 \mathrm{~m}(15 \mathrm{~mL})$. The aqueous layers were combined and extracted with $\mathrm{Et}_{2} \mathrm{O}(20$ $\mathrm{mL})$. The organic layers were combined, washed with saturated $\mathrm{NaHCO}_{3}(20 \mathrm{~mL})$, brine $(20 \mathrm{~mL})$, dried with $\mathrm{MgSO}_{4}$ and concentrated under reduced pressure.

\section{3-((Triiso-propylsilyl)ethynyl)-1H-indole (3a)}

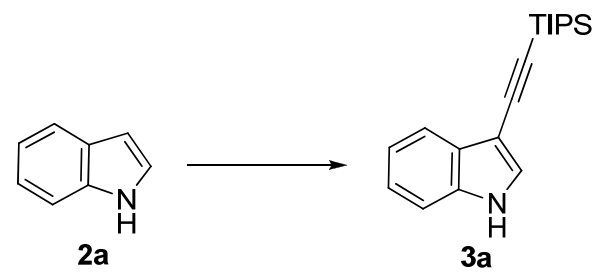

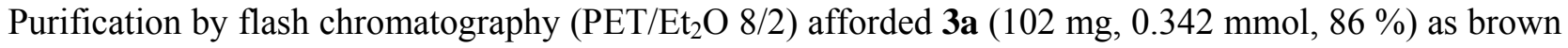
solid. Rf0.4 (PET/Et ${ }_{2} \mathrm{O}$ 7:3, UV/Anisaldehyde).

Large scale procedure: 1d (1.0 g, $2.4 \mathrm{mmol}, 1.2$ equiv) was added to a stirring solution of $\mathrm{AuCl}$ (4.6 mg, $0.020 \mathrm{mmol}, 0.01$ equiv) and 2a (0.24 g, $2.0 \mathrm{mmol}, 1.0$ equiv) in $\mathrm{Et}_{2} \mathrm{O}(40 \mathrm{~mL})$ under air. The reaction was sealed and stirred at room temperature for $12-15 \mathrm{~h} . \mathrm{Et}_{2} \mathrm{O}(50 \mathrm{~mL})$ was added, the organic layer was washed twice with $\mathrm{NaOH} 0.1 \mathrm{M}(50 \mathrm{~mL})$. The aqueous layers were combined and extracted with $\mathrm{Et}_{2} \mathrm{O}(50 \mathrm{~mL})$. The organic layers were combined, washed with saturated $\mathrm{NaHCO}_{3}(50 \mathrm{~mL})$, brine $(50 \mathrm{~mL})$, dried with $\mathrm{MgSO}_{4}$ and concentrated under reduced pressure. Purification by flash chromatography $\left(\mathrm{PET} / \mathrm{Et}_{2} \mathrm{O} 8 / 2\right)$ afforded $3 a$ (498 mg, $1.68 \mathrm{mmol}, 84 \%$ ) as brown solid.

$\mathrm{Rf} 0.4\left(\mathrm{PET} / \mathrm{Et}_{2} \mathrm{O}\right.$ 7/3, UV/Anisaldehyde);

$\operatorname{Mp} 55-58^{\circ} \mathrm{C}$; 
${ }^{1} \mathrm{H}$ NMR $\left(\mathrm{CDCl}_{3}, 400 \mathrm{MHz}\right) \delta 8.11($ br s, $1 \mathrm{H} ; \mathrm{NH}), 7.79(\mathrm{~m}, 1 \mathrm{H} ; \mathrm{ArH}), 7.40(\mathrm{~d}, J(H, H)=2.7 \mathrm{~Hz}, 1 \mathrm{H}$; ArH), 7.36 (m, 1 H), 7.26 (m, 2 H; ArH), 1.22 (m, 21 H; TIPS);

${ }^{13} \mathrm{C} \mathrm{NMR}\left(\mathrm{CDCl}_{3}, 100 \mathrm{MHz}\right) \delta 135.1,128.9,128.3,123.1,120.8,120.1,111.4,100.4,99.3,92.19,18.8$, 11.5

IR v $3407(\mathrm{~m}), 3062(\mathrm{w}), 2942$ (s), $2891(\mathrm{~m}), 2864$ (s), 2152 (s), 1620 (w), $1532(\mathrm{w}), 1457(\mathrm{~s}), 1416(\mathrm{~m})$, 1383 (w), 1341 (w), 1325 (m), 1239 (s), 1128 (m), 1071 (m), 996 (m), 910 (m), 883 (s), 774 (s), 742 (s), 676 (s), $658(\mathrm{~s}), 628(\mathrm{~s})$;

HRMS(ESI) calcd for $\mathrm{C}_{19} \mathrm{H}_{28} \mathrm{NSi}^{+}$(M+H) 298.1991, found 298.2001.

2-Iodobenzoic acid was recovered by adjusting the $\mathrm{pH}$ of the $\mathrm{NaOH}$ fraction to 1 with conc. $\mathrm{HCl}$ and extracting with $\mathrm{CH}_{2} \mathrm{Cl}_{2}(3 \times 100 \mathrm{~mL})$. The organic layers were combined, dried over $\mathrm{MgSO}_{4}$ and concentrated under reduced pressure to afford 2-Iodobenzoic acid (375 $\mathrm{mg}, 1.45 \mathrm{mmol}, 63 \%$ recovered) as a colorless solid.

\section{1-Methyl-3-((triiso-propylsilyl)ethynyl)-1H-indole (3b)}

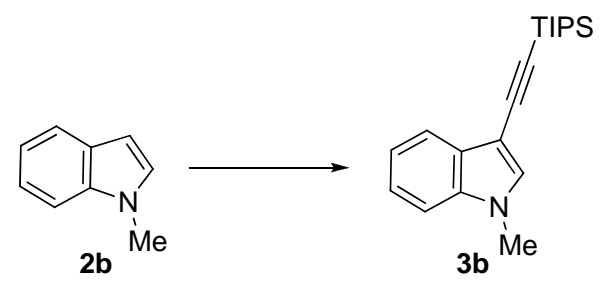

Purification by flash chromatography ( $\mathrm{PET} / \mathrm{Et}_{2} \mathrm{O}$ 99/1) afforded $3 \mathbf{b}(103 \mathrm{mg}, 0.331 \mathrm{mmol}, 83 \%)$ as yellow solid.

$\mathrm{Rf} 0.6\left(\mathrm{PET} / \mathrm{Et}_{2} \mathrm{O}\right.$ 99:1, UV/Anisaldehyde);

$\operatorname{Mp~60-61~}{ }^{\circ}$;

${ }^{1} \mathrm{H}$ NMR $\left(\mathrm{CDCl}_{3}, 400 \mathrm{MHz}\right) \delta 7.81(\mathrm{dd}, J(H, H)=0.3,7.4 \mathrm{~Hz}, 1 \mathrm{H} ; \mathrm{ArH}), 7.36-7.24(\mathrm{~m}, 4 \mathrm{H} ; \mathrm{ArH}), 3.78$ (s, $\left.3 \mathrm{H} ; \mathrm{CH}_{3}\right), 1.26$ (m, $21 \mathrm{H}$; TIPS);

${ }^{13} \mathrm{C} \mathrm{NMR}\left(\mathrm{CDCl}_{3}, 100 \mathrm{MHz}\right) \delta 136.0,132.6,129.6,122.6,120.3,120.1,109.4,100.5,97.5,91.4,32.9$, $18.8,11.4$

IR v $3129(\mathrm{w}), 3051(\mathrm{w}), 2957$ (s), 2939 (s), 2889 (m), 2863 (s), 2721(w), $2365(\mathrm{w}), 2144$ (s), $1715(\mathrm{w})$, 1616 (w), 1538 (m), 1462 (s), 1424 (w), 1381 (s), 1349 (m), 1328 (m), 1249 (m), 1162 (m), 1130 (m), 1075 (m), $1016(\mathrm{~m}), 992(\mathrm{~m}), 919(\mathrm{w}), 883(\mathrm{~s}), 804(\mathrm{~m}), 768(\mathrm{~m}), 727$ (s), 705 (s), $674(\mathrm{~s}), 648(\mathrm{~s}), 608(\mathrm{~m})$;

HRMS(ESI) calcd for $\mathrm{C}_{20} \mathrm{H}_{30} \mathrm{NSi}^{+}(\mathrm{M}+\mathrm{H}) 312.2148$, found 312.2162.

\section{5-Methoxy-3-((triiso-propylsilyl)ethynyl)-1H-indole (3c)}

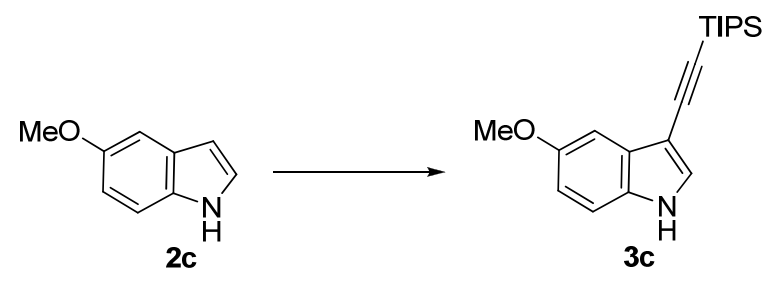


Purification by flash chromatography (PET/Et $2 \mathrm{O} 8 / 2)$ afforded 3c (105 mg, $0.321 \mathrm{mmol}, 80$ \%) as brown amorphous solid.

$\mathrm{Rf} 0.3\left(\mathrm{PET} / \mathrm{Et}_{2} \mathrm{O}\right.$ 8/2, UV/Anisaldehyde);

${ }^{1} \mathrm{H}$ NMR $\left(\mathrm{CDCl}_{3}, 400 \mathrm{MHz}\right) \delta 8.18($ br s, $1 \mathrm{H} ; \mathrm{NH}), 7.37(\mathrm{~d}, J(H, H)=2.6 \mathrm{~Hz}, 1 \mathrm{H} ; \mathrm{ArH}), 7.27(\mathrm{~d}$, $J(H, H)=2.2 \mathrm{~Hz}, 1 \mathrm{H} ; \mathrm{ArH}), 7.24(\mathrm{~d}, J(H, H)=8.8 \mathrm{~Hz}, 1 \mathrm{H} ; \mathrm{ArH}), 6.93(\mathrm{dd}, J(H, H)=2.4,8.8 \mathrm{~Hz}, 1 \mathrm{H} ; \mathrm{ArH})$, 3.93 (s, $\left.3 \mathrm{H} ; \mathrm{CH}_{3}\right), 1.24$ (m, $21 \mathrm{H}$; TIPS);

${ }^{13} \mathrm{C} \mathrm{NMR}\left(\mathrm{CDCl}_{3}, 100 \mathrm{MHz}\right) \delta 154.8,130.0,129.7,128.6,113.3,112.2,101.4,100.5,98.8,92.0,55.6$, $18.7,11.4$

IR $v 3473(\mathrm{w}), 3394(\mathrm{w}), 2945$ (w), $2892(\mathrm{w}), 2867(\mathrm{w}), 2253(\mathrm{w}), 2155$ (w), 1625 (w), 1587 (w), 1486 (w), $1464(\mathrm{w}), 1439(\mathrm{w}), 1288(\mathrm{w}), 1249(\mathrm{w}), 1213(\mathrm{w}), 1177(\mathrm{w}), 1062(\mathrm{w}), 1026(\mathrm{w}), 907(\mathrm{~s}), 884(\mathrm{w}), 838$ (w), $802(\mathrm{w}), 731(\mathrm{~s}), 676(\mathrm{w}), 651(\mathrm{~m}), 644(\mathrm{w})$;

HRMS(ESI) calcd for $\mathrm{C}_{20} \mathrm{H}_{30} \mathrm{NOSi}^{+}(\mathrm{M}+\mathrm{H})$ 328.2097, found 328.2090.

\section{3-((Triiso-propylsilyl)ethynyl)-1H-indol-5-ol (3d)}

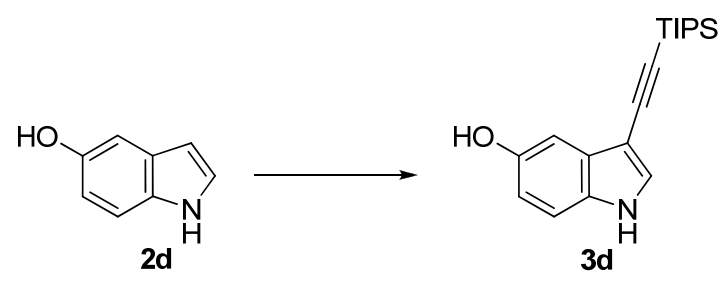

Following the general procedure except that the work up was modified as following: $\mathrm{Et}_{2} \mathrm{O}(10 \mathrm{~mL})$ was added to the reaction mixture, the organic layer was washed twice with $\mathrm{NaHCO}_{3}(15 \mathrm{~mL})$. The aqueous layers were combined and extracted with $\mathrm{Et}_{2} \mathrm{O}(20 \mathrm{~mL})$. The organic layers were combined, dried with $\mathrm{MgSO}_{4}$ and concentrated under reduced pressure. Purification by flash chromatography (PET/Et $2 \mathrm{O} 7 / 3$ ) afforded 3d (96 mg, $0.31 \mathrm{mmol}, 76 \%$ ) as a tan solid.

$\mathrm{Rf} 0.3\left(\mathrm{PET} / \mathrm{Et}_{2} \mathrm{O} 7 / 3, \mathrm{UV} /\right.$ Anisaldehyde);

$\operatorname{Mp} 124-126^{\circ} \mathrm{C}$;

${ }^{1} \mathrm{H}$ NMR $\left(\mathrm{CDCl}_{3}, 400 \mathrm{MHz}\right) \delta 8.10($ br s, $1 \mathrm{H} ; \mathrm{NH}), 7.40(\mathrm{~d}, J(H, H)=2.6 \mathrm{~Hz}, 1 \mathrm{H} ; \mathrm{ArH}), 7.22(\mathrm{~d}$, $J(H, H)=8.8 \mathrm{~Hz}, 1 \mathrm{H} ; \mathrm{ArH}), 7.16(\mathrm{~d}, J(H, H)=2.2 \mathrm{~Hz}, 1 \mathrm{H} ; \mathrm{ArH}), 6.84(\mathrm{dd}, J(H, H)=2.4,8.8 \mathrm{~Hz}, 1 \mathrm{H} ; \operatorname{ArH})$, 4.87 (br s, $1 \mathrm{H} ; \mathrm{OH}), 1.19$ (s, $21 \mathrm{H}$; TIPS);

${ }^{13} \mathrm{C} \mathrm{NMR}\left(\mathrm{CDCl}_{3}, 100 \mathrm{MHz}\right) \delta 150.4,130.3,129.9,129.2,112.9,112.1,104.6,100.2,98.8,91.8,18.8$, 11.5

IR v 3407 (m), 2943 (s), 2865 (s), 2147 (m), $1627(\mathrm{w}), 1585$ (w), 1489 (m), 1463 (s), 1419 (w), 1365 (w), 1315 (w), 1245 (m), $1191(\mathrm{~m}), 1102$ (w), 1053 (m), 997 (w), 936 (m), 936 (m), 884 (m), 837 (m), 799 (m), $740(\mathrm{~s}), 678(\mathrm{~s})$;

HRMS(ESI) calcd for $\mathrm{C}_{19} \mathrm{H}_{28} \mathrm{NOSi}^{+}(\mathrm{M}+\mathrm{H}) 314.1940$, found 314.1925. 

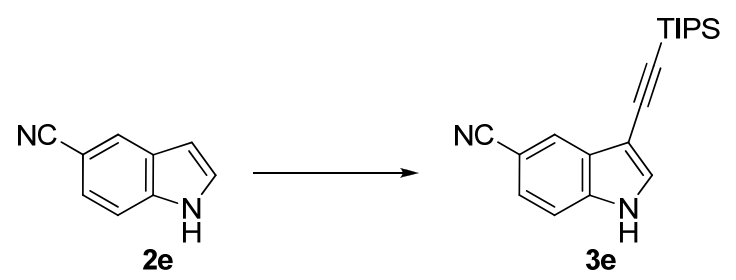

Purification by flash chromatography (PET/Et ${ }_{2} \mathrm{O}$ 6/4) afforded 3e (103 mg, 0.319 mmol, 80 \%) as tan solid.

$\mathrm{R} f$ 0.25(PET/Et $2 \mathrm{O} 6 / 4, \mathrm{UV} /$ Anisaldehyde);

$\operatorname{Mp} 164-167^{\circ} \mathrm{C}$;

${ }^{1} \mathrm{H}$ NMR $\left(\mathrm{CDCl}_{3}, 400 \mathrm{MHz}\right) \delta 9.06($ br s, $1 \mathrm{H} ; \mathrm{NH}), 8.09(\mathrm{~d}, J(H, H)=0.7 \mathrm{~Hz}, 1 \mathrm{H} ; \mathrm{ArH}), 7.58(\mathrm{~d}$, $J(H, H)=2.6 \mathrm{~Hz}, 1 \mathrm{H} ; \mathrm{ArH}), 7.50$ (m, $2 \mathrm{H}$; ArH), 1.20 (s, $21 \mathrm{H}$; TIPS);

${ }^{13} \mathrm{C} \mathrm{NMR}\left(\mathrm{CDCl}_{3}, 100 \mathrm{MHz}\right) \delta 136.9,130.5,128.8,125.9,125.6,120.7,112.6,103.5,100.3,98.4,93.6$, $18.8,11.4$

IR v $3282(\mathrm{~m}), 2941(\mathrm{~m}), 2864(\mathrm{~m}), 2226(\mathrm{~s}), 2153(\mathrm{~s}), 1620(\mathrm{~m}), 1464(\mathrm{~m}), 1432(\mathrm{~m}), 1361(\mathrm{w}), 1288$ (w), $1244(\mathrm{~s}), 1178(\mathrm{~m}), 1104(\mathrm{~m}), 1073(\mathrm{w}), 996(\mathrm{~m}), 910$ (s), $883(\mathrm{~s}), 822(\mathrm{~m}), 804(\mathrm{~m}), 769(\mathrm{~m}), 737$ (s), $718(\mathrm{~s}), 672(\mathrm{~s}), 638(\mathrm{~s})$;

HRMS(ESI) calcd for $\mathrm{C}_{20} \mathrm{H}_{27} \mathrm{~N}_{2} \mathrm{Si}^{+}(\mathrm{M}+\mathrm{H}) 323.1944$, found 323.1952 .

\section{3-((Triiso-propylsilyl)ethynyl)-1H-indole-5-carboxylic acid (3f)}

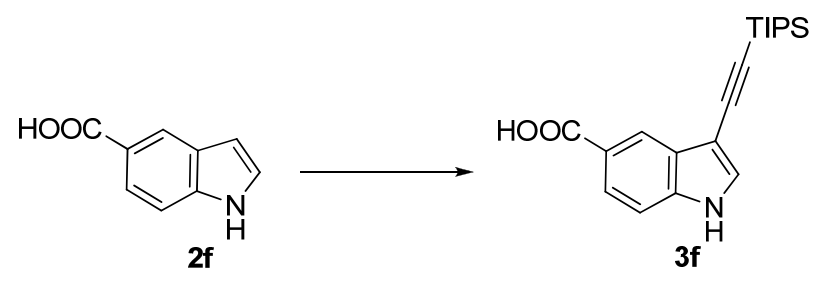

Following the general procedure except that the work up was modified as following: the reaction mixture was concentrated and the crude mixture purified by flash chromatography ( $\mathrm{PET}_{\mathrm{Et}} \mathrm{O}$ 4/6) affording $3 \mathbf{f}$ (92 $\mathrm{mg}, 0.27 \mathrm{mmol}, 67 \%$ ) as a colorless solid.

$\mathrm{Rf} 0.3\left(\mathrm{PET} / \mathrm{Et}_{2} \mathrm{O}\right.$ 4/6, UV/Anisaldehyde);

$\mathrm{Mp} 157-160^{\circ} \mathrm{C}$

${ }^{1} \mathrm{H}$ NMR (MeOD, 400MHz) $\delta 8.42(\mathrm{~m}, 1 \mathrm{H} ; \mathrm{ArH}), 7.89$ (dd, $\left.J(H, H)=1.7,8.6 \mathrm{~Hz}, 1 \mathrm{H} ; \mathrm{ArH}\right), 7.56$ (s, 1 $\mathrm{H} ; \mathrm{ArH}), 7.44(\mathrm{dd}, J(H, H)=0.5,8.6 \mathrm{~Hz}, 1 \mathrm{H}$; ArH), $1.16(\mathrm{~m}, 21 \mathrm{H})$;

${ }^{13} \mathrm{C}$ NMR (MeOD, 100MHz) $\delta 171.1,139.6,131.6,129.8,125.0,123.6,123.6,112.5,101.8,100.6,92.3$, $19.2,12.6$ 
IR v $3370(\mathrm{~m}), 2943(\mathrm{~s}), 2874(\mathrm{~m}), 2865(\mathrm{~s}), 2501(\mathrm{~m}), 2245(\mathrm{w}), 2151(\mathrm{~m}), 2075(\mathrm{w}), 1680(\mathrm{~s}), 1616$ (m), 1575 (w), 1463 (m), 1417 (m), 1355 (m), 1313 (m), 1267 (s), 1242 (s), 1119 (m), 1072 (m), 977 (m), $917(\mathrm{~m}), 884(\mathrm{~s}), 818(\mathrm{~s}), 772(\mathrm{~s}), 753(\mathrm{~s}), 671(\mathrm{~s})$;

HRMS(ESI) calcd for $\mathrm{C}_{20} \mathrm{H}_{28} \mathrm{NO}_{2} \mathrm{Si}^{+}(\mathrm{M}+\mathrm{H}) 342.1889$, found 342.1890 .

\section{5-Nitro-3-((triiso-propylsilyl)ethynyl)-1H-indole (3g)}

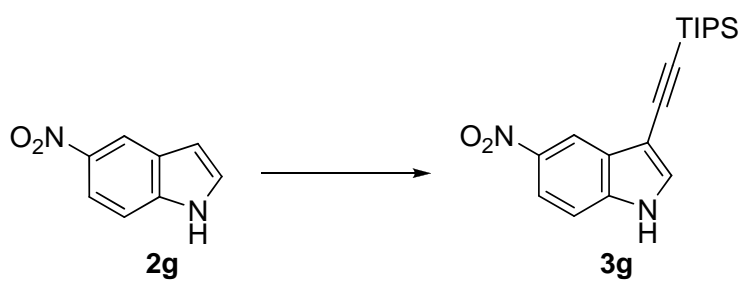

Purification by flash chromatography (PET/Et $2 \mathrm{O} 7 / 3)$ afforded $3 g(116 \mathrm{mg}, 0.339 \mathrm{mmol}, 85 \%)$ as yellow solid as an inseparable mixture $95 / 5$ with 5-nitroindole.

$\mathrm{R} f 0.3\left(\mathrm{PET} / \mathrm{Et}_{2} \mathrm{O} 7 / 3, \mathrm{UV} /\right.$ Anisaldehyde);

$\operatorname{Mp~} 193-195^{\circ} \mathrm{C}$;

${ }^{1} \mathrm{H}$ NMR $\left(\mathrm{CDCl}_{3}, 400 \mathrm{MHz}\right) \delta 9.10($ br s, $1 \mathrm{H} ; \mathrm{NH}), 8.70(\mathrm{~d}, J(H, H)=2.1 \mathrm{~Hz}, 1 \mathrm{H} ; \mathrm{ArH}), 8.17$ (dd, $J(H, H)=2.1,8.9 \mathrm{~Hz}, 1 \mathrm{H} ; \mathrm{ArH}), 7.63(\mathrm{~d}, J(H, H)=2.6 \mathrm{~Hz}, 1 \mathrm{H} ; \mathrm{ArH}), 7.50(\mathrm{~d}, J(H, H)=8.9 \mathrm{~Hz}, 1 \mathrm{H} ; \operatorname{ArH})$, 1.21 (s, $21 \mathrm{H}$; TIPS);

${ }^{13} \mathrm{C} \mathrm{NMR}\left(\mathrm{CDCl}_{3}, 100 \mathrm{MHz}\right) \delta 142.4,138.1,131.3,128.5,118.6,117.3,111.8,102.0,98.1,94.3,18.8$, 11.4

IR v $3319(\mathrm{~m}), 2943(\mathrm{~m}), 2865(\mathrm{~m}), 2153(\mathrm{~m}), 1621(\mathrm{w}), 1463(\mathrm{~m}), 1329(\mathrm{~s}), 1282(\mathrm{~m}), 1244(\mathrm{~m}), 1113$ (m), 1041 (w), 995 (w), 909 (s), 883 (m), 843 (w), $818(w), 796$ (m), 769 (m), 736 (s), 675 (s);

HRMS(ESI) calcd for $\mathrm{C}_{19} \mathrm{H}_{27} \mathrm{O}_{2} \mathrm{~N}_{2} \mathrm{Si}^{+}(\mathrm{M}+\mathrm{H}) 343.1842$, found 343.1835.

\section{5-Bromo-3-((triiso-propylsilyl)ethynyl)-1H-indole (3h)}
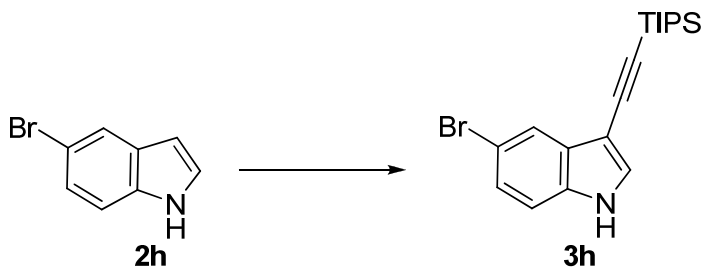

Purification by flash chromatography (PET/Et ${ }_{2} \mathrm{O}$ 8/2) afforded $3 \mathbf{h}(140 \mathrm{mg}, 0.372 \mathrm{mmol}, 93$ \%) as brown amorphous solid.

Rf 0.3 (PET/EtOAc 7/3, UV/Anisaldehyde);

${ }^{1} \mathrm{H}$ NMR $\left(\mathrm{CDCl}_{3}, 400 \mathrm{MHz}\right) \delta 8.19($ br s, $1 \mathrm{H} ; \mathrm{NH}), 7.85(\mathrm{dd}, J(H, H)=0.5,1.2 \mathrm{~Hz}, 1 \mathrm{H}$; ArH), $7.38(\mathrm{~d}$, $J(H, H)=2.7 \mathrm{~Hz}, 1 \mathrm{H} ; \mathrm{ArH}), 7.32(\mathrm{dd}, J(H, H)=1.9,8.6 \mathrm{~Hz}, 1 \mathrm{H} ; \mathrm{ArH}), 7.20(\mathrm{~d}, J(H, H)=8.6 \mathrm{~Hz}, 1 \mathrm{H} ; \operatorname{ArH})$, 1.19 (m, $21 \mathrm{H}$; TIPS); 
${ }^{13} \mathrm{C} \mathrm{NMR}\left(\mathrm{CDCl}_{3}, 100 \mathrm{MHz}\right) \delta 133.6,130.5,129.2,126.0,122.6,114.1,112.8,99.3,99.1,92.6,18.7$, 11.4

IR v $3539(\mathrm{w}), 3464(\mathrm{~m}), 3387(\mathrm{~m}), 2960$ (m), 2941 (m), 2864 (m), 2150 (m), 1649 (w), $1567(\mathrm{w}), 1526$ (w), $1462(\mathrm{~s}), 1415(\mathrm{w}), 1273(\mathrm{~m}), 1238(\mathrm{~m}), 1102(\mathrm{w}), 1063(\mathrm{w}), 998(\mathrm{w}), 908(\mathrm{~s}), 884(\mathrm{~s}), 796(\mathrm{~s}), 733$ (s), $670(\mathrm{~s}), 652(\mathrm{~s})$;

HRMS(ESI) calcd for $\mathrm{C}_{19} \mathrm{H}_{27}{ }^{79} \mathrm{BrNSi}^{+}(\mathrm{M}+\mathrm{H}) 376.1096$, found 378.1103 .

\section{5-Iodo-3-((triiso-propylsilyl)ethynyl)-1H-indole (3i)}

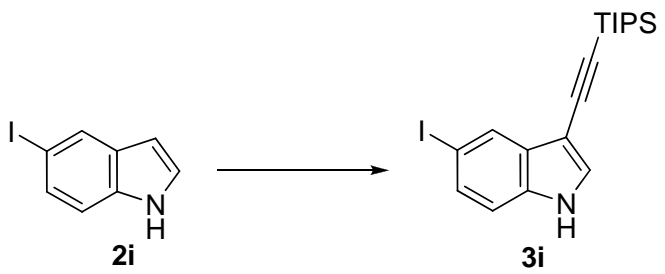

Purification by flash chromatography (PET/Et $2 \mathrm{O} 7 / 3$ ) afforded 3i (154 mg, $0.363 \mathrm{mmol}, 91 \%$ ) as brown solid.

$\mathrm{Rf} 0.2\left(\mathrm{PET} / \mathrm{Et}_{2} \mathrm{O} 7 / 3, \mathrm{UV} /\right.$ Anisaldehyde);

$\operatorname{Mp} 75-77^{\circ} \mathrm{C}$;

${ }^{1} \mathrm{H} \mathrm{NMR}\left(\mathrm{CDCl}_{3}, 400 \mathrm{MHz}\right) \delta 8.20($ br s, $1 \mathrm{H} ; \mathrm{NH}), 8.09(\mathrm{~d}, J(H, H)=1.0 \mathrm{~Hz}, 1 \mathrm{H} ; \mathrm{ArH}), 7.51$ (dd, $J(H, H)=1.5,8.4 \mathrm{~Hz}, \mathrm{~Hz}, 1 \mathrm{H}$; ArH), $7.35(\mathrm{~d}, J(H, H)=2.6 \mathrm{~Hz}, 1 \mathrm{H} ; \mathrm{ArH}), 7.12(\mathrm{~d}, J(H, H)=8.6 \mathrm{~Hz}, 1 \mathrm{H}$; ArH), 1.22 (m, $21 \mathrm{H}$; TIPS);

${ }^{13} \mathrm{C} \mathrm{NMR}\left(\mathrm{CDCl}_{3}, 100 \mathrm{MHz}\right) \delta 134.1,131.6,131.3,129.0,128.9,113.4,99.4,98.8,92.7,84.5,18.9$, 11.5

IR v $3388(\mathrm{~m}), 2941(\mathrm{~s}), 2863$ (s), 2152 (m), $1644(\mathrm{w}), 1527$ (w), 1450 (s), 1413 (m), 1334 (w), 1272 (m), $1240(\mathrm{~m}), 1139(\mathrm{w}), 110(\mathrm{w}), 1074(\mathrm{~m}), 996(\mathrm{w}), 909$ (m), 880 (s), 795 (s), 736 (s), 666 (s);

HRMS(ESI) calcd for $\mathrm{C}_{19} \mathrm{H}_{27} \mathrm{INSi}^{+}(\mathrm{M}+\mathrm{H})$ 424.0958, found 424.0943.

\section{4-Bromo-3-((triiso-propylsilyl)ethynyl)-1H-indole (3j)}
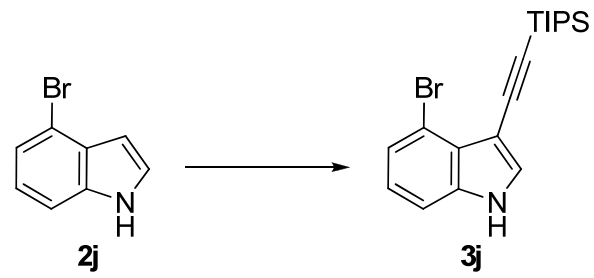

Purification by flash chromatography (PET/Et $\left.{ }_{2} \mathrm{O} 7 / 3\right)$ afforded $\mathbf{3 j}(120 \mathrm{mg}, 0.319 \mathrm{mmol}, 80 \%$ ) as brown solid as an inseparable $96 / 4$ mixture with 4-bromoindole.

$\mathrm{R} f 0.3\left(\mathrm{PET} / \mathrm{Et}_{2} \mathrm{O}\right.$ 7/3, UV/Anisaldehyde);

$\operatorname{Mp~} 73-74{ }^{\circ} \mathrm{C}$; 
${ }^{1} \mathrm{H}$ NMR $\left(\mathrm{CDCl}_{3}, 400 \mathrm{MHz}\right) \delta 8.29$ (br s, $\left.1 \mathrm{H} ; \mathrm{NH}\right), 7.46(\mathrm{~d}, J(H, H)=2.7 \mathrm{~Hz}, 1 \mathrm{H} ; \mathrm{ArH}), 7.34(\mathrm{~m}, 1 \mathrm{H}$; ArH), 7.27 (m, $1 \mathrm{H}$; ArH), 7.04 (t, $J(H, H)=7.7 \mathrm{~Hz}, 1 \mathrm{H}$; ArH), 1.21 (m, $21 \mathrm{H}$; TIPS);

${ }^{13} \mathrm{C} \mathrm{NMR}\left(\mathrm{CDCl}_{3}, 100 \mathrm{MHz}\right) \delta 135.9,131.2,126.1,125.2,123.9,115.4,110.8,100.4,100.0,93.5,18.8$, 11.7 ;

IR v $3398(\mathrm{~m}), 2940(\mathrm{~m}), 2863(\mathrm{~m}), 2148(\mathrm{~m}), 1612(\mathrm{w}), 1561(\mathrm{w}), 1525(\mathrm{w}), 1462(\mathrm{~m}), 1416(\mathrm{~m}), 1336$ (w), $1297(\mathrm{~m}), 1240(\mathrm{w}), 1192(\mathrm{~m}), 1143(\mathrm{w}), 1108(\mathrm{w}), 1076(\mathrm{w}), 1044(\mathrm{w}), 998(\mathrm{w}), 911(\mathrm{~m}), 882(\mathrm{~m}), 772$ (s), $738(\mathrm{~s}), 673(\mathrm{~s}), 661(\mathrm{~s}), 636(\mathrm{~m})$;

HRMS(ESI) calcd for $\mathrm{C}_{19} \mathrm{H}_{27}{ }^{79} \mathrm{BrNSi}^{+}(\mathrm{M}+\mathrm{H}) 376.1096$, found 376.1075.

\section{6-Bromo-3-((triiso-propylsilyl)ethynyl)-1H-indole (3k)}

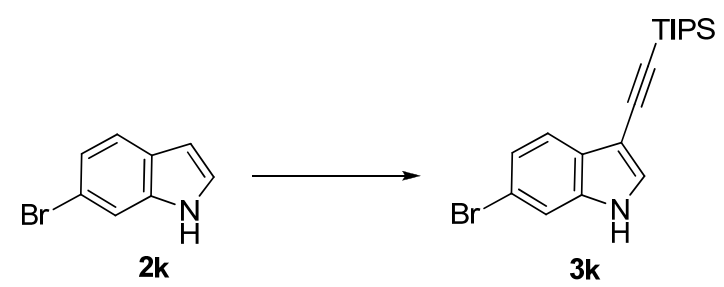

Purification by flash chromatography (PET/Et $\left.{ }_{2} \mathrm{O} 85 / 15\right)$ afforded 3k (115 mg, 0.306 mmol, $77 \%$ ) as brown oil.

$\mathrm{Rf} 0.4\left(\mathrm{PET} / \mathrm{Et}_{2} \mathrm{O}\right.$ 8/2, UV/Anisaldehyde);

${ }^{1} \mathrm{H}$ NMR $\left(\mathrm{CDCl}_{3}, 400 \mathrm{MHz}\right) \delta 8.16($ br s, $1 \mathrm{H} ; \mathrm{NH}), 7.63(\mathrm{~d}, J(H, H)=8.4 \mathrm{~Hz}, 1 \mathrm{H} ; \mathrm{ArH}), 7.48(\mathrm{~d}$, $J(H, H)=1.5 \mathrm{~Hz}, 1 \mathrm{H} ; \mathrm{ArH}), 7.38(\mathrm{~d}, J(H, H)=2.6 \mathrm{~Hz}, 1 \mathrm{H} ; \mathrm{ArH}), 7.35(\mathrm{dd}, J(H, H)=1.4,8.4 \mathrm{~Hz}, 1 \mathrm{H} ; \mathrm{ArH})$, 1.22 (s, $21 \mathrm{H}$; TIPS);

${ }^{13} \mathrm{C} \mathrm{NMR}\left(\mathrm{CDCl}_{3}, 100 \mathrm{MHz}\right) \delta 135.7,128.7,127.7,124.1,121.2,116.5,114.3,113.9,99.4,92.6,18.7$, 11.3

IR v $3418(\mathrm{~m}), 3123(\mathrm{w}), 2943(\mathrm{~s}), 2874(\mathrm{~m}), 2864(\mathrm{~s}), 2152(\mathrm{~m}), 1706(\mathrm{w}), 1614(\mathrm{~m}), 1531(\mathrm{w}), 1452$ (m), 1391 (m), 1318 (m), 1239 (m), 1136 (m), 1099 (m), 1069 (s), 996 (m), 920 (w), 895 (m), 883 (s), 848 (w), $803(\mathrm{~s}), 775(\mathrm{~s}), 728(\mathrm{w}), 670(\mathrm{~s})$;

HRMS(ESI) calcd for $\mathrm{C}_{19} \mathrm{H}_{27}{ }^{79} \mathrm{BrNSi}^{+}(\mathrm{M}+\mathrm{H}) 376.1096$, found 376.1111.

\section{7-Bromo-3-((triiso-propylsilyl)ethynyl)-1H-indole (3I)}

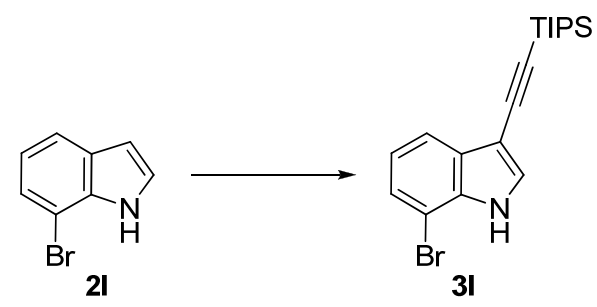

Purification by flash chromatography (PET/Et ${ }_{2} \mathrm{O}$ 97/3) afforded 31 (125 mg, $\left.0.332 \mathrm{mmol}, 84 \%\right)$ as colorless solid.

$\mathrm{R} f 0.4\left(\mathrm{PET} / \mathrm{Et}_{2} \mathrm{O}\right.$ 7/3, UV/Anisaldehyde); 


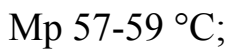

${ }^{1} \mathrm{H}$ NMR $\left(\mathrm{CDCl}_{3}, 400 \mathrm{MHz}\right) \delta 8.35($ br s, $1 \mathrm{H} ; \mathrm{NH}), 7.70(\mathrm{~d}, J(H, H)=7.9 \mathrm{~Hz}, 1 \mathrm{H} ; \mathrm{ArH}), 7.50(\mathrm{~d}$, $J(H, H)=2.6 \mathrm{~Hz}, 1 \mathrm{H}$; ArH), $7.41(\mathrm{~m}, 1 \mathrm{H} ; \mathrm{ArH}), 7.11(\mathrm{t}, J(H, H)=7.7 \mathrm{~Hz}, 1 \mathrm{H}$; ArH), 1.19 (s, $21 \mathrm{H}$; TIPS);

${ }^{13} \mathrm{C} \mathrm{NMR}\left(\mathrm{CDCl}_{3}, 100 \mathrm{MHz}\right) \delta 133.9,130.1,128.6,125.4,121.9,119.4,104.8,100.9,99.5,92.5,18.8$, 11.4;

IR v 3401 (s), 3117 (w), 2942(s), 2864 (s), 2146 (m), 1564 (w), $1489(\mathrm{w}), 1430$ (m), 1404 (m), $1382(\mathrm{w})$, 1318 (m), 1238 (m), 1201 (m), 1139 (w), 1077 (m), 997 (m), 910 (w), 883 (s), 832 (s), 769 (s), 736 (s), 676 (s), $664(\mathrm{~s}), 619(\mathrm{~m})$;

HRMS(ESI) calcd for $\mathrm{C}_{19} \mathrm{H}_{27}{ }^{79} \mathrm{BrNSi}^{+}(\mathrm{M}+\mathrm{H}) 376.1096$, found 376.1102.

\section{2-Methyl-3-((triiso-propylsilyl)ethynyl)-1H-indole (3m)}

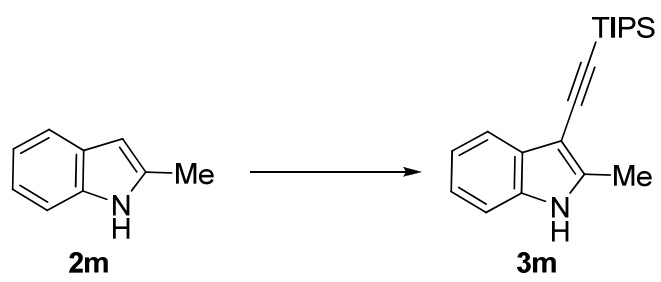

Purification by flash chromatography (PET/Et $\left.{ }_{2} \mathrm{O} 7 / 3\right)$ afforded $3 \mathrm{~m}(112 \mathrm{mg}, 0.360 \mathrm{mmol}, 90 \%)$ as brown amorphous solid.

$\mathrm{Rf} 0.3\left(\mathrm{PET} / \mathrm{Et}_{2} \mathrm{O}\right.$ 7:3, UV/Anisaldehyde);

${ }^{1} \mathrm{H}$ NMR $\left(\mathrm{CDCl}_{3}, 400 \mathrm{MHz}\right) \delta 7.75$ (br s, $\left.1 \mathrm{H} ; \mathrm{NH}\right), 7.71$ (m, $\left.1 \mathrm{H} ; \mathrm{ArH}\right), 7.28-7.17$ (m, $\left.3 \mathrm{H} ; \mathrm{ArH}\right), 2.48$ (s, $\left.3 \mathrm{H} ; \mathrm{CH}_{3}\right), 1.25$ (m, $21 \mathrm{H}$; TIPS);

${ }^{13} \mathrm{C} \mathrm{NMR}\left(\mathrm{CDCl}_{3}, 100 \mathrm{MHz}\right) \delta 140.3,134.5,129.3,122.1,120.5,119.2,110.6,100.7,96.9,93.2,18.8$, 12.6, 11.4;

IR v $3462(\mathrm{w}), 3394(\mathrm{~m}), 2943(\mathrm{~m}), 2865(\mathrm{~m}), 2251(\mathrm{w}), 2147(\mathrm{~m}), 1461(\mathrm{~m}), 1430(\mathrm{w}), 1307(\mathrm{~m}), 1243$ (m), $1161(\mathrm{w}), 1072(\mathrm{w}), 1013(\mathrm{~m}), 997$ (m), 908 (s), 883 (m), 777 (m), 734 (s), 673 (m), 653 (m), 628 (m);

HRMS(ESI) calcd for $\mathrm{C}_{20} \mathrm{H}_{30} \mathrm{NSi}^{+}(\mathrm{M}+\mathrm{H}) 312.2148$, found 312.2150.

\section{2-Phenyl-3-((triiso-propylsilyl)ethynyl)-1H-indole (3n)}

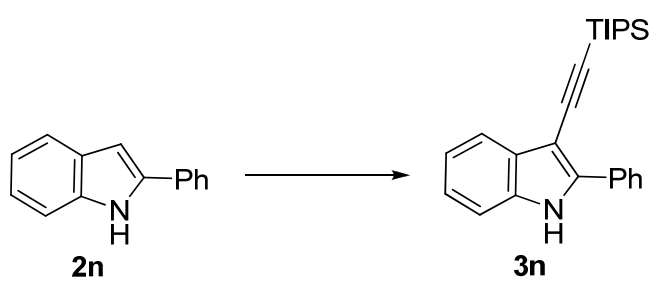

Purification by flash chromatography (PET/Et $\left.{ }_{2} \mathrm{O} 85 / 15\right)$ afforded $3 n$ (132 mg, $\left.0.353 \mathrm{mmol}, 88 \%\right)$ as slightly yellow amorphous solid.

$\mathrm{Rf} 0.4\left(\mathrm{PET} / \mathrm{Et}_{2} \mathrm{O}\right.$ 8:2, UV/Anisaldehyde). 
${ }^{1} \mathrm{H}$ NMR $\left(\mathrm{CDCl}_{3}, 400 \mathrm{MHz}\right) \delta 8.32$ (br s, $\left.1 \mathrm{H} ; \mathrm{NH}\right), 8.11$ (m, $\left.2 \mathrm{H} ; \mathrm{ArH}\right), 7.80$ (m, $\left.1 \mathrm{H} ; \mathrm{ArH}\right), 7.49$ (m, 2 H; ArH), 7.40 (m, 2 H; ArH), 7.27 (m, 2H; ArH), 1.24 (s, 21 H; TIPS);

${ }^{13} \mathrm{C}$ NMR $\left(\mathrm{CDCl}_{3}, 100 \mathrm{MHz}\right) \delta 139.7,135.1,131.3,130.8,128.7,128.3,126.4,123.4,120.9,120.1$, $110.9,101.2,96.5,95.1,18.8,11.5$;

IR $v 3420(\mathrm{w}), 3060(\mathrm{w}), 2942$ (m), $2874(\mathrm{~m}), 2864(\mathrm{~m}), 2141(\mathrm{~m}), 1604(\mathrm{w}), 1489(\mathrm{w}), 1459(\mathrm{~m})$, 1327(m), 1237(m), 1177(w), 998(m), 908(s), 883(m), 787(m), 768(m), 740(s), 691(s), 676(s), 660(s), 633(m);

HRMS(ESI) calcd for $\mathrm{C}_{25} \mathrm{H}_{32} \mathrm{NSi}^{+}(\mathrm{M}+\mathrm{H})$ 374.2304, found 374.2307.

\section{(3-((Triiso-propylsilyl)ethynyl)-1H-indol-2-yl)methanol (3o)}

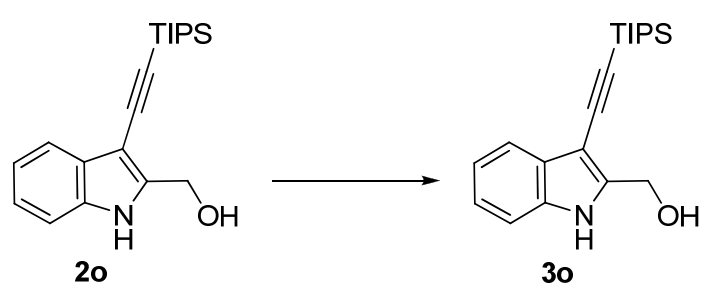

Purification by flash chromatography $\left(\mathrm{PET} \mathrm{Et}_{2} \mathrm{O}\right.$ 1/1) afforded 3 o (107 mg, $\left.0.326 \mathrm{mmol}, 82 \%\right)$ as orange solid.

$\mathrm{Rf} 0.4\left(\mathrm{PET} / \mathrm{Et}_{2} \mathrm{O} 1 / 1, \mathrm{UV} /\right.$ Anisaldehyde);

$\operatorname{Mp} 93-94^{\circ} \mathrm{C}$;

${ }^{1} \mathrm{H}$ NMR $\left(\mathrm{CDCl}_{3}, 400 \mathrm{MHz}\right) \delta 8.65$ (br s, $\left.1 \mathrm{H} ; \mathrm{NH}\right), 7.71$ (m, $\left.1 \mathrm{H} ; \mathrm{ArH}\right), 7.24$ (m, $\left.3 \mathrm{H} ; \mathrm{ArH}\right), 4.94$ (d, $\left.J(H, H)=2.6 \mathrm{~Hz}, 2 \mathrm{H} ; \mathrm{CH}_{2}\right), 2.71$ (br s, $\left.1 \mathrm{H} ; \mathrm{OH}\right), 1.21$ (m, $21 \mathrm{H}$; TIPS);

${ }^{13} \mathrm{C} \mathrm{NMR}\left(\mathrm{CDCl}_{3}, 100 \mathrm{MHz}\right) \delta 141.5,135.0,129.0,123.2,120.8,119.4,111.4,99.4,96.6,94.6,57.3$, $18.8,11.4$

IR v $3538(\mathrm{~m}), 3273(\mathrm{~m}), 2940(\mathrm{~m}), 2864(\mathrm{~m}), 2147(\mathrm{~m}), 1460(\mathrm{~m}), 1390(\mathrm{w}), 1327(\mathrm{~m}), 1246(\mathrm{~m}), 1187$ (w), $1001(\mathrm{~m}), 968(\mathrm{~m}), 910(\mathrm{~m}), 883(\mathrm{~m}), 782(\mathrm{~s}), 736(\mathrm{~s}), 677$ (s), $659(\mathrm{~s}), 628(\mathrm{~s})$;

HRMS(ESI) calcd for $\mathrm{C}_{20} \mathrm{H}_{30} \mathrm{NOSi}^{+}(\mathrm{M}+\mathrm{H}) 328.2097$, found 328.2104.

\section{3-Methyl-2-((triiso-propylsilyl)ethynyl)-1H-indole (3p)}

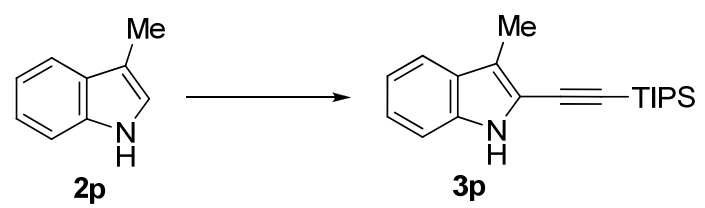

Purification by flash chromatography ( $\mathrm{PET} / \mathrm{Et}_{2} \mathrm{O}$ 99/1) afforded 3p (95 mg, $0.31 \mathrm{mmol}, 76 \%$ ) as amorphous orange solid.

$\mathrm{Rf} 0.1\left(\mathrm{PET} / \mathrm{Et}_{2} \mathrm{O}\right.$ 99/1, UV/Anisaldehyde);

$\operatorname{Mp} 75-77^{\circ} \mathrm{C}$; 
${ }^{1} \mathrm{H}$ NMR $\left(\mathrm{CDCl}_{3}, 400 \mathrm{MHz}\right) \delta 7.98($ br s, $1 \mathrm{H} ; \mathrm{NH}), 7.59(\mathrm{~d}, J(H, H)=7.9 \mathrm{~Hz}, 1 \mathrm{H} ; \mathrm{ArH}), 7.28(\mathrm{~m}, 2 \mathrm{H}$; ArH), 7.17 (m, $1 \mathrm{H}$; ArH), 2.47 (d, $J(H, H)=0.5 \mathrm{~Hz}, 3 \mathrm{H}$; Me), 1.23 (m, $21 \mathrm{H}$; TIPS);

${ }^{13} \mathrm{C} \mathrm{NMR}\left(\mathrm{CDCl}_{3}, 100 \mathrm{MHz}\right) \delta 135.6,127.8,123.7,119.8,119.3,118.9,117.0,110.7,98.5,97.4,18.8$, $11.4,9.6$

IR v $3478(\mathrm{~m}), 3415(\mathrm{~m}), 3059(\mathrm{w}), 2942(\mathrm{~m}), 2865(\mathrm{~m}), 2145(\mathrm{~m}), 1455(\mathrm{~m}), 1383(\mathrm{w}), 1362(\mathrm{~m}), 1338$ (m), 1243 (w), $1154(\mathrm{w}), 1115(\mathrm{w}), 1073(\mathrm{w}), 997$ (m), 909 (w), $883(\mathrm{~m}), 858(\mathrm{w}), 733$ (s), $710(\mathrm{~m}), 676$ (s);

HRMS(ESI) calcd for $\mathrm{C}_{20} \mathrm{H}_{30} \mathrm{NSi}^{+}(\mathrm{M}+\mathrm{H}) 312.2148$, found 312.2160.

2-((Triiso-propylsilyl)ethynyl)-1H-pyrrole (6a)

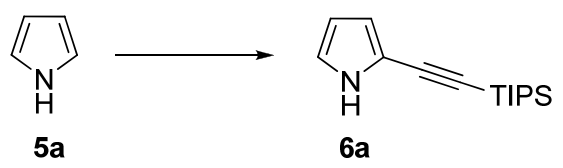

Following the general procedure: purification by flash chromatography (PET/Et $\left.{ }_{2} \mathrm{O} 98 / 2\right)$ afforded $6 a$ (62 $\mathrm{mg}, 0.25 \mathrm{mmol}, 62 \%$ ) as green oil.

Following general procedure but using 5a $(84 \mu \mathrm{L}, 1.2 \mathrm{mmol}, 3$ equiv) and 1d (171 mg, $0.400 \mathrm{mmol}, 1$ equiv): purification by flash chromatography (PET/Et $\left.{ }_{2} \mathrm{O} 98 / 2\right)$ afforded $6 a(82 \mathrm{mg}, 0.33 \mathrm{mmol}, 83 \%)$ as green oil.

$\mathrm{Rf} 0.3\left(\mathrm{PET} / \mathrm{EtOAc} 98: 2, \mathrm{UV} / \mathrm{KMnO}_{4}\right)$;

${ }^{1} \mathrm{H}$ NMR $\left(\mathrm{CDCl}_{3}, 400 \mathrm{MHz}\right) \delta 8.36$ (br s, $\left.1 \mathrm{H}, \mathrm{NH}\right), 6.19(\mathrm{td}, J(H, H)=2.7,3.4 \mathrm{~Hz}, 1 \mathrm{H}, \mathrm{ArH}), 6.51$ (ddd, $J(H, H)=1.5,2.4,3.8 \mathrm{~Hz}, 1 \mathrm{H}, \mathrm{ArH}), 6.75(\mathrm{dt}, J(H, H)=1.4,2.7, \mathrm{~Hz}, 1 \mathrm{H}, \mathrm{ArH}), 1.14-1.13$ (m, 21H, TIPS).

${ }^{13} \mathrm{C} \mathrm{NMR}\left(\mathrm{CDCl}_{3}, 400 \mathrm{MHz}\right) \delta 119.1,118.7,115.3,109.1,99.0,91.9,18.7,11.4 ;$

IR v $3478(\mathrm{~m}), 3405(\mathrm{~m}), 2943$ (s), 2865 (s), 2364 (w), 2146 (s), 1716 (w), 1550 (w), 1463 (m), 1384 (w), $1254(\mathrm{w}), 1119(\mathrm{~m}), 1119(\mathrm{~m}), 1094(\mathrm{~m}), 1034(\mathrm{~m}), 997$ (m), 962 (m), 911 (m), 883 (s), 804 (m), 755 (s), $728(\mathrm{~s}), 676(\mathrm{~s}), 658(\mathrm{~s})$;

HRMS(ESI) calcd for $\mathrm{C}_{15} \mathrm{H}_{26} \mathrm{NSi}^{+}(\mathrm{M}+\mathrm{H}) 248.1835$, found 248.1847.

1-Methyl-2-((triiso-propylsilyl)ethynyl)-1H-pyrrole (6b), 1-Methyl-3-((triiso-propylsilyl)ethynyl)-1Hpyrrole (7b)

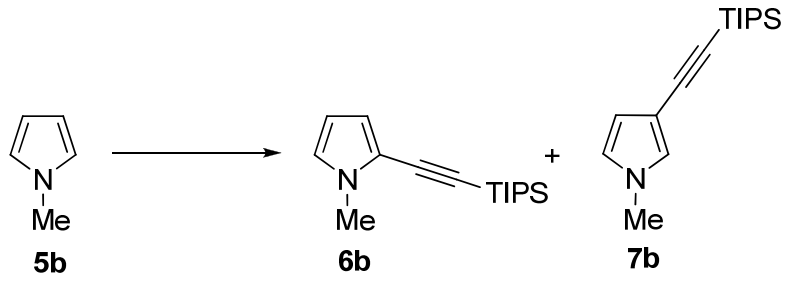

Following the general procedure but using $5 \mathbf{b}(107 \mu \mathrm{L}, 1.20 \mathrm{mmol}, 3$ equiv) and $\mathbf{1 d}(171 \mathrm{mg}, 0.400$ mmol, 1 equiv): purification by flash chromatography (PET) afforded $6 \mathbf{b}$ (50 mg, $0.19 \mathrm{mmol}, 48 \%$ ) as orange amorphous solid and 7b (26 mg, $0.099 \mathrm{mmol}, 25 \%)$ as violet amorphous solid.

6b: 
$\mathrm{Rf} 0.1\left(\mathrm{PET}, \mathrm{UV} / \mathrm{KMnO}_{4}\right)$;

${ }^{1} \mathrm{H}$ NMR $\left(\mathrm{CDCl}_{3}, 400 \mathrm{MHz}\right) \delta 6.62(\mathrm{dd}, J(H, H)=1.9,2.6 \mathrm{~Hz}, 1 \mathrm{H} ; \mathrm{ArH}), 6.44(\mathrm{~m}, 1 \mathrm{H} ; \mathrm{ArH}), 6.06(\mathrm{~m}, 1$ $\mathrm{H}$; ArH), $3.69\left(\mathrm{~d}, J(H, H)=0.3 \mathrm{~Hz}, 3 \mathrm{H} ; \mathrm{CH}_{3}\right), 1.14$ (m, $21 \mathrm{H}$; TIPS);

${ }^{13} \mathrm{C} \mathrm{NMR}\left(\mathrm{CDCl}_{3}, 100 \mathrm{MHz}\right) \delta 125.5,123.4,115.0,107.8,98.4,94.5,34.6,18.7,11.4$

IR v $3108(\mathrm{w}), 3108(\mathrm{~s}), 2943(\mathrm{~m}), 2892(\mathrm{~s}), 2865(\mathrm{w}), 2724(\mathrm{w}), 2363(\mathrm{w}), 2145(\mathrm{~s}), 1716(\mathrm{w}), 1645(\mathrm{w})$, $1572(\mathrm{w}), 1528(\mathrm{w}), 1463(\mathrm{~m}), 1420(\mathrm{w}), 1383$ (w), 1366 (w), $1310(\mathrm{~m}), 1233(\mathrm{~m}), 1177$ (m), $1073(\mathrm{w}), 1053$ (m), $1014(\mathrm{~m}), 996(\mathrm{~m}), 918(\mathrm{~m}), 883$ (s), 780 (s), 717 (s), 689 (s), 675 (s), 661 (s);

HRMS(ESI) calcd for $\mathrm{C}_{16} \mathrm{H}_{28} \mathrm{NSi}^{+}(\mathrm{M}+\mathrm{H})$ 262.1991, found 262.1994.

7b:

$\mathrm{R} f 0.4\left(\mathrm{PET}, \mathrm{UV} / \mathrm{KMnO}_{4}\right)$;

${ }^{1} \mathrm{H}$ NMR $\left(\mathrm{CDCl}_{3}, 400 \mathrm{MHz}\right) \delta 6.83(\mathrm{~m}, 1 \mathrm{H} ; \mathrm{ArH}), 6.47(\mathrm{t}, J(H, H)=2.2 \mathrm{~Hz}, 1 \mathrm{H} ; \mathrm{ArH}), 6.24(\mathrm{~m}, 1 \mathrm{H}$; ArH), 3.61 (s, $\left.3 \mathrm{H} ; \mathrm{CH}_{3}\right), 1.10$ (m, $21 \mathrm{H}$; TIPS);

${ }^{13} \mathrm{C} \mathrm{NMR}\left(\mathrm{CDCl}_{3}, 100 \mathrm{MHz}\right) \delta 126.3,121.5,112.5,104.8,102.6,87.5,36.3,18.7,11.5$;

IR v 3127 (w), 2942 (s), 2892 (m), 2865 (s), 2722 (w), $2364(\mathrm{w}), 2342(\mathrm{w}), 2145(\mathrm{~m}), 1716(\mathrm{w}), 1543$ (w), $1509(\mathrm{w}), 1463(\mathrm{~m}), 1422(\mathrm{w}), 1382(\mathrm{w}), 1364(\mathrm{~m}), 1346(\mathrm{~m}), 1231(\mathrm{w}), 1170(\mathrm{w}), 1075(\mathrm{w}), 982(\mathrm{~m})$, $918(\mathrm{w}), 883$ (s), 779 (s), 764 (s), 700 (m), 668 (s), 641 (s);

HRMS(ESI) calcd for $\mathrm{C}_{16} \mathrm{H}_{28} \mathrm{NSi}^{+}(\mathrm{M}+\mathrm{H}) 262.1991$, found 262.1990 .

\section{1-(Triiso-propylsilyl)-3-((triiso-propylsilyl)ethynyl)-1H-pyrrole (7c)}

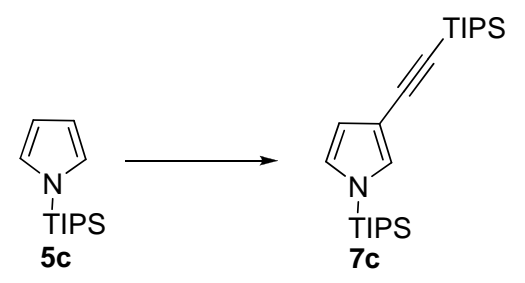

Purification by flash chromatography (PET/Et ${ }_{2} \mathrm{O}$ 98/2) afforded 7c (127 mg, $\left.0.315 \mathrm{mmol}, 79 \%\right)$ as yellow oil.

$\mathrm{R} f 0.5\left(\mathrm{PET} / \mathrm{Et}_{2} \mathrm{O} 99 / 1, \mathrm{UV} / \mathrm{KMnO}_{4}\right)$.

${ }^{1} \mathrm{H}$ NMR $\left(\mathrm{CDCl}_{3}, 400 \mathrm{MHz}\right) \delta 7.01(\mathrm{~m}, 1 \mathrm{H} ; \mathrm{ArH}), 6.67(\mathrm{t}, J(H, H)=2.6 \mathrm{~Hz}, 1 \mathrm{H} ; \mathrm{ArH}), 6.43(\mathrm{dd}$, $J(H, H)=1.0,2.4 \mathrm{~Hz}, 1 \mathrm{H}$; ArH), 1.47 (m, $3 \mathrm{H}$; TIPS), 1.14 (m, $39 \mathrm{H}$; TIPS);

${ }^{13} \mathrm{C} \mathrm{NMR}\left(\mathrm{CDCl}_{3}, 100 \mathrm{MHz}\right) \delta 128.9,123.9,114.4,106.9,103.0,88.1,18.8,17.8,11.6,11.5$;

IR v $2945(\mathrm{~m}), 2866(\mathrm{~m}), 2362(\mathrm{w}), 2151(\mathrm{~m}), 1463(\mathrm{~m}), 1385$ (w), $1296(\mathrm{~m}), 1222(\mathrm{w}), 1097(\mathrm{~s}), 1074$ (m), 1017 (m), 985 (m), 909 (s), 883 (s), 790 (m), 735 (s), 674 (s), 659 (s);

HRMS(ESI) calcd for $\mathrm{C}_{24} \mathrm{H}_{46} \mathrm{NSi}_{2}{ }^{+}(\mathrm{M}+\mathrm{H})$ 404.3169, found 404.3155. 
2-Ethyl-5-((triiso-propylsilyl)ethynyl)-1H-pyrrole (6d)

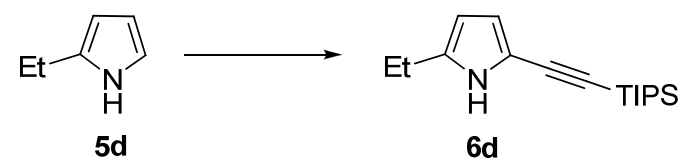

Purification by flash chromatography (PET -> PET/ $\mathrm{CH}_{2} \mathrm{Cl}_{2}$ 99/1) afforded 6d (64 mg, 0.23 mmol, 58 $\%)$ as colorless oil. Turning then green without impurities appearing in NMR.

$\mathrm{Rf} 0.1\left(\mathrm{PET} / \mathrm{CH}_{2} \mathrm{Cl}_{2}\right.$ 99/1, UV/KMnO 4$)$;

${ }^{1} \mathrm{H} \mathrm{NMR}\left(\mathrm{CDCl}_{3}, 400 \mathrm{MHz}\right) \delta 8.04$ (br s, $\left.1 \mathrm{H} ; \mathrm{NH}\right), 6.40(\mathrm{dd}, J(H, H)=2.9,3.4 \mathrm{~Hz}, 1 \mathrm{H} ; \mathrm{ArH}), 5.86(\mathrm{~m}, 1$ $\mathrm{H} ; \mathrm{ArH}), 2.62\left(\mathrm{q}, J(H, H)=7.7 \mathrm{~Hz}, 2 \mathrm{H} ; \mathrm{CH}_{2}\right), 1.25\left(\mathrm{t}, J(H, H)=7.6 \mathrm{~Hz}, 3 \mathrm{H} ; \mathrm{CH}_{3}\right), 1.12$ (m, $21 \mathrm{H}$; TIPS);

${ }^{13} \mathrm{C} \mathrm{NMR}\left(\mathrm{CDCl}_{3}, 100 \mathrm{MHz}\right) \delta 135.9,115.8,111.7,105.5,99.5,91.3,21.0,18.7,13.5,11.4$;

IR v $3472(\mathrm{w}), 3384(\mathrm{w}), 2943$ (s), 2865 (s), $2141(\mathrm{~s}), 1572$ (w), 1463 (m), $1386(\mathrm{w}), 1330(\mathrm{w}), 995(\mathrm{~m})$, $882(\mathrm{~m}), 761(\mathrm{~s}), 676(\mathrm{~s}), 657(\mathrm{~s})$;

HRMS(ESI) calcd for $\mathrm{C}_{17} \mathrm{H}_{30} \mathrm{NSi}^{+}(\mathrm{M}+\mathrm{H})$ 276.2148, found 276.2158.

\section{2-Phenyl-5-((triiso-propylsilyl)ethynyl)-1H-pyrrole (6e)}

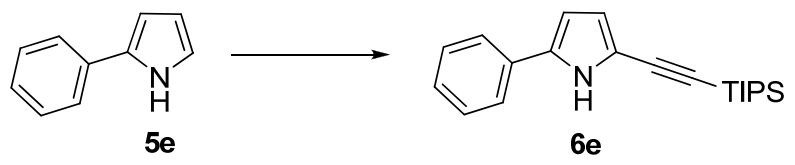

Purification by flash chromatography (PET/DCM 95/5) afforded 6e (79 mg, $0.24 \mathrm{mmol}, 60 \%)$ as pink amorphous solid.

$\mathrm{Rf} 0.2\left(\mathrm{PET} / \mathrm{Et}_{2} \mathrm{O} 5 / 95, \mathrm{UV} / \mathrm{KMnO}_{4}\right)$;

${ }^{1} \mathrm{H}$ NMR $\left(\mathrm{CDCl}_{3}, 400 \mathrm{MHz}\right) \delta 8.54$ ( br s, $\left.1 \mathrm{H} ; \mathrm{NH}\right), 7.52(\mathrm{~m}, 2 \mathrm{H} ; \mathrm{PhH}), 7.41(\mathrm{~m}, 2 \mathrm{H} ; \mathrm{PhH}), 7.28(\mathrm{~m}, 1$ $\mathrm{H} ; \mathrm{PhH}), 6.60(\mathrm{dd}, J(H, H)=2.2,3.4 \mathrm{~Hz}, 1 \mathrm{H} ; \mathrm{PyrH}), 6.50(\mathrm{dd}, J(H, H)=2.7,3.6 \mathrm{~Hz}, 1 \mathrm{H} ; \mathrm{PyrH}), 1.19(\mathrm{~m}, 21$ $\mathrm{H}$; TIPS);

${ }^{13} \mathrm{C} \mathrm{NMR}\left(\mathrm{CDCl}_{3}, 100 \mathrm{MHz}\right) \delta 133.1,131.9,129.0,126.9,124.2,117.3,114.3,106.8,99.0,93.1,18.8$, 11.4;

IR v $3471(\mathrm{w}), 3424(\mathrm{w}), 2942(\mathrm{~m}), 2864(\mathrm{~m}), 2142(\mathrm{~m}), 1604(\mathrm{w}), 1505(\mathrm{~m}), 1461(\mathrm{~m}), 1383(\mathrm{w}), 1305$ (w), $1243(\mathrm{w}), 1073(\mathrm{w}), 1044(\mathrm{w}), 996(\mathrm{~m}), 892(\mathrm{w}), 882(\mathrm{~m}), 781(\mathrm{~m}), 755(\mathrm{~s}), 677(\mathrm{~s})$;

HRMS(ESI) calcd for $\mathrm{C}_{21} \mathrm{H}_{30} \mathrm{NSi}^{+}(\mathrm{M}+\mathrm{H})$ 324.2148, found 324.2139.

1-(5-((Triiso-propylsilyl)ethynyl)-1H-pyrrol-3-yl)ethanone (6f)

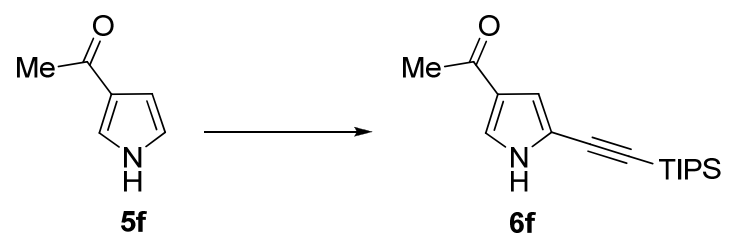

Purification by flash chromatography (PET/Et $2 \mathrm{O} 7 / 3)$ afforded $\mathbf{6 f}(67 \mathrm{mg}, 0.23 \mathrm{mmol}, 58 \%)$ as tan solid. 
$\mathrm{R} f 0.3\left(\mathrm{PET} / \mathrm{Et}_{2} \mathrm{O} 7 / 3, \mathrm{UV} / \mathrm{KMnO}_{4}\right)$;

$\operatorname{Mp} 120-122^{\circ} \mathrm{C}$;

${ }^{1} \mathrm{H}$ NMR $\left(\mathrm{CDCl}_{3}, 400 \mathrm{MHz}\right) \delta 9.52($ br s, $1 \mathrm{H} ; \mathrm{NH}), 7.37(\mathrm{dd}, J(H, H)=1.2,2.6 \mathrm{~Hz}, \mathrm{ArH}), 6.87(\mathrm{~m}, 1 \mathrm{H}$; ArH), 2.44 (s, $3 \mathrm{H}$; Ac), 1.10 (m, $21 \mathrm{H}$; TIPS);

${ }^{13} \mathrm{C} \mathrm{NMR}\left(\mathrm{CDCl}_{3}, 100 \mathrm{MHz}\right) \delta 193.7,126.2,124.1,115.3,115.1,97.4,93.4,27.3,18.6,11.3$;

IR v $3554(\mathrm{~m}), 3223(\mathrm{~m}), 2866(\mathrm{~m}), 2151(\mathrm{~m}), 1645(\mathrm{~s}), 1562(\mathrm{w}), 1496(\mathrm{~m}), 1464(\mathrm{~m}), 1394(\mathrm{~m}), 1342$ (m), 1247 (w), 1204 (m), 1149 (w), 1077 (w), 998 (w), 935 (m), $884(\mathrm{~m}), 832$ (m), 767 (m), $738(\mathrm{~m}), 660$ $(\mathrm{m})$;

HRMS(ESI) calcd for $\mathrm{C}_{17} \mathrm{H}_{28} \mathrm{ONSi}^{+}(\mathrm{M}+\mathrm{H})$ 290.1940, found 290.1938.

\section{2,5-Dimethyl-3-((triiso-propylsilyl)ethynyl)-1H-pyrrole (7g)}

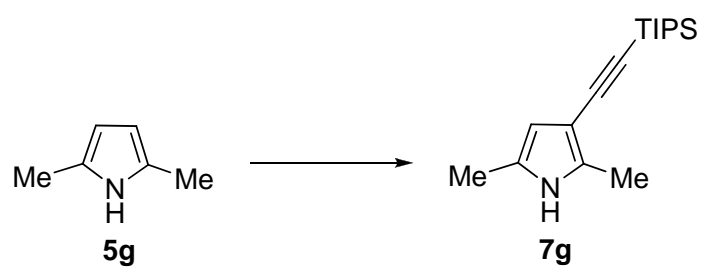

Purification by flash chromatography ( $\mathrm{PET} \mathrm{Et}_{2} \mathrm{O}$ 85/15) afforded $\mathbf{7 g}(65 \mathrm{mg}, 0.24 \mathrm{mmol}, 59 \%$ ) as yellow oil.

$\mathrm{Rf} 0.3\left(\mathrm{PET} / \mathrm{Et}_{2} \mathrm{O} 85: 15, \mathrm{UV} / \mathrm{KMnO}_{4}\right)$;

${ }^{1} \mathrm{H}$ NMR $\left(\mathrm{CDCl}_{3}, 400 \mathrm{MHz}\right) \delta 7.58(\mathrm{br} \mathrm{s}, 1 \mathrm{H} ; \mathrm{NH}), 5.90(\mathrm{~d}, J(H, H)=1.7 \mathrm{~Hz}, 1 \mathrm{H} ; \mathrm{ArH}), 2.32(\mathrm{~s}, 3 \mathrm{H}$; Me), 2.20 (s, $3 \mathrm{H}$; Me), 1.14 (m, $21 \mathrm{H}$; TIPS);

${ }^{13} \mathrm{C} \mathrm{NMR}\left(\mathrm{CDCl}_{3}, 100 \mathrm{MHz}\right) \delta 132.3,125.5,108.8,103.1,102.3,89.1,18.8,12.8,12.0,11.5 ;$

IR v 3374 (m), 2942 (s), 2865 (s), 2146 (s), 1594 (w), 1463 (m), 1384 (w), 1371 (w), 1307 (m), 1244 (w), $1157(\mathrm{w}), 1127(\mathrm{w}), 1074(\mathrm{w}), 996(\mathrm{~m}), 919(\mathrm{w}), 882(\mathrm{~s}), 812(\mathrm{~s}), 788(\mathrm{~s}), 702$ (s), $675(\mathrm{~s}), 658(\mathrm{~s})$;

HRMS(ESI) calcd for $\mathrm{C}_{17} \mathrm{H}_{30} \mathrm{NSi}^{+}(\mathrm{M}+\mathrm{H}) 276.2148$ found 276.2150 .

\section{3-Ethyl-2,4-dimethyl-5-((triiso-propylsilyl)ethynyl)-1H-pyrrole (6h)}

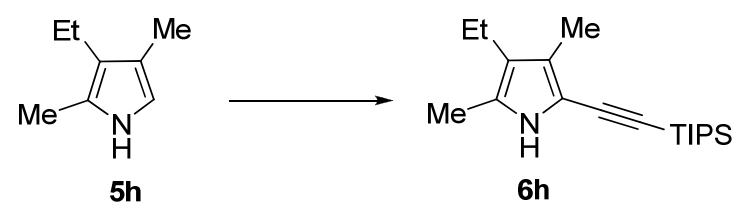

Purification by flash chromatography (PET/DCM 99/1) afforded $6 \mathbf{h}$ (58 mg, 0.19 mmol, 48 \%) as yellow oil.

$\mathrm{Rf} 0.25\left(\mathrm{PET} / \mathrm{CH}_{2} \mathrm{Cl}_{2}\right.$ 99/1, UV/KMnO 4$)$;

${ }^{1} \mathrm{H}$ NMR $\left(\mathrm{CDCl}_{3}, 400 \mathrm{MHz}\right) \delta 7.74$ (br s, $\left.1 \mathrm{H} ; \mathrm{NH}\right), 2.40\left(\mathrm{q}, J(H, H)=7.6 \mathrm{~Hz}, 2 \mathrm{H} ; \mathrm{CH}_{2}\right), 2.19$ (s, $3 \mathrm{H}$; Me), 2.14 (s, $3 \mathrm{H}$; Me), 1.15 (m, $21 \mathrm{H}$; TIPS), 1.08 (t, $J(H, H)=7.7 \mathrm{~Hz}, 3 \mathrm{H} ; \mathrm{CH}_{2} \mathrm{CH}_{3}$ ); 
${ }^{13} \mathrm{C} \mathrm{NMR}\left(\mathrm{CDCl}_{3}, 100 \mathrm{MHz}\right) \delta 125.5,124.7,121.4,108.9,99.4,93.6,18.8,17.6,15.4,11.4,11.2,10.0$;

IR v 3478 (w), 3398 (w), 2960 (s), 2942 (s), 2865 (s), 2133 (s), 1587 (w), 1462 (m), 1382 (w), 1329 (w), $1309(\mathrm{w}), 1243(\mathrm{w}), 1138(\mathrm{w}), 1066(\mathrm{w}), 1016(\mathrm{w}), 996(\mathrm{~m}), 916(\mathrm{~m}), 883$ (s), 851 (m), $775(\mathrm{w}), 724(\mathrm{~m})$, $703(\mathrm{~m}), 674(\mathrm{~s}), 658(\mathrm{~s})$;

HRMS(ESI) calcd for $\mathrm{C}_{19} \mathrm{H}_{34} \mathrm{NSi}^{+}(\mathrm{M}+\mathrm{H}) 304.2461$, found 304.2453 .

\section{Deprotection procedure:}

\section{3-Ethynyl-1H-indole (14)}
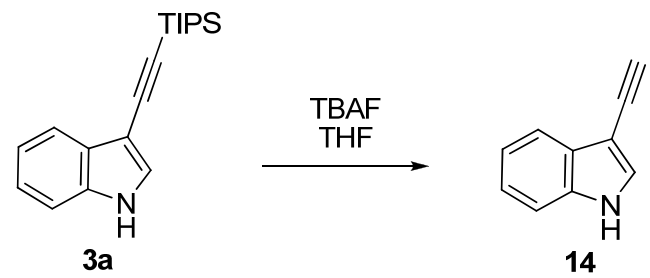

Tetrabutylammonium fluoride ( $1 \mathrm{M}$ in THF, $0.80 \mathrm{~mL}, 0.80 \mathrm{mmol}, 2.0$ equiv) was added to a stirring solution of 3a (119 mg, $0.400 \mathrm{mmol}, 1$ equiv) in THF $(5 \mathrm{ml})$. The reaction was stirred at room temperature for $15 \mathrm{~h}$. The reaction was quenched with a saturated solution of $\mathrm{NH}_{4} \mathrm{Cl}(10 \mathrm{~mL})$ and extracted twice with $\mathrm{Et}_{2} \mathrm{O}(15 \mathrm{~mL})$. The organic layers were combined, washed with saturated $\mathrm{NaHCO}_{3}(20 \mathrm{~mL})$, brine $(20 \mathrm{~mL})$, dried with $\mathrm{MgSO}_{4}$ and concentrated under reduced pressure. The crude mixture was purified by flash chromatography (hexane/Et $2 \mathrm{O} 7 / 3$ ) to afford 14 (53 $\mathrm{mg}, 0.38 \mathrm{mmol}, 94 \%)$ as a tan solid.

$\mathrm{Rf} 0.25$ (hexane/ $\mathrm{Et}_{2} \mathrm{O} 7 / 3, \mathrm{UV} / \mathrm{KMnO}_{4}$ );

$\operatorname{Mp~} 109-111^{\circ} \mathrm{C}$;

${ }^{1} \mathrm{H}$ NMR $\left(\mathrm{CDCl}_{3}, 400 \mathrm{MHz}\right) \delta 8.16($ br s, $1 \mathrm{H} ; \mathrm{NH}), 7.82(\mathrm{~d}, J(H, H)=7.4 \mathrm{~Hz}, 1 \mathrm{H} ; \mathrm{ArH}), 7.45(\mathrm{~d}$, $J(H, H)=1.7 \mathrm{~Hz}, 1 \mathrm{H}$; ArH), 7.39 (m, $1 \mathrm{H}$; ArH), 7.28 (m, $2 \mathrm{H}$; ArH), 3.28 (s, $1 \mathrm{H}$; acetylene);

${ }^{13} \mathrm{C} \mathrm{NMR}\left(\mathrm{CDCl}_{3}, 100 \mathrm{MHz}\right) \delta 134.9,128.8,128.5,123.2,120.9,119.9,111.4,97.5,78.8,77.4 ;$

${ }^{13} \mathrm{C}$ DEPT $135 \mathrm{NMR}\left(\mathrm{CDCl}_{3}, 100 \mathrm{MHz}\right) \delta: 128.9,123.3,120.9,119.9,111.5,78.9,77.5$;

IR v: 3407(s), $3266(\mathrm{~s}), 3125(\mathrm{w}), 3052(\mathrm{w}), 2926(\mathrm{w}), 2852(\mathrm{w}), 2106(\mathrm{w}), 1653(\mathrm{w}), 1618(\mathrm{w}), 1526$ (w), $1458(\mathrm{~m}), 1421(\mathrm{~m}), 1362(\mathrm{w}), 1342(\mathrm{w}), 1325(\mathrm{w}), 1242$ (m), $1126(\mathrm{w}), 1104(\mathrm{w}), 1091(\mathrm{w}), 1061$ (w), $1005(\mathrm{w}), 912(\mathrm{w}), 829(\mathrm{w}), 767(\mathrm{w}), 756(\mathrm{w}), 744(\mathrm{~m}), 714(\mathrm{~m}), 685(\mathrm{w}), 676(\mathrm{w}), 649(\mathrm{w}), 635(\mathrm{~m})$;

HRMS(ESI) calcd for $\mathrm{C}_{10} \mathrm{H}_{8} \mathrm{~N}^{+}(\mathrm{M}+\mathrm{H})$ 142.0657, found 142.0674 .

The data are consistent with reported data ${ }^{[7]}$ excepted the ${ }^{13} \mathrm{C}$ peak reported at $122.5 \mathrm{ppm}$ by authors which has not been observed in our case. In contrast the peak at $77.4 \mathrm{ppm}$ was not reported maybe due to the proximity of $\mathrm{CDCl}_{3}$ signal. DEPT 135 confirmed the presence of this signal, which can be attributed to one of the acetylene carbons.

DEPT experiments (see spectra) was not conclusive to differentiate definitively the two acetylene carbons at 78.8 and $77.4 \mathrm{ppm}$, as even the quaternary carbon displayed a significant signal in all DEPT 
experiments. To confirm further the assignment, $\mathbf{1 4}$ was deprotonated with $n \mathrm{BuLi}$ in THF and the resulting organolithium was quenched with $\mathrm{D}_{2} \mathrm{O}$. The obtained C-D coupling was much larger at $78.8 \mathrm{ppm}(38.2 \mathrm{~Hz}$ vs $7.7 \mathrm{~Hz}$ ), confirming definitively this carbon to be the terminal one.

${ }^{1} \mathrm{H}$ NMR $\left(\mathrm{CDCl}_{3}, 400 \mathrm{MHz}\right) \delta 8.15$ (br s, $\left.1 \mathrm{H} ; \mathrm{NH}\right), 7.84(\mathrm{~d}, J(H, H)=6.9 \mathrm{~Hz}, 1 \mathrm{H} ; \mathrm{ArH}), 7.41(\mathrm{~d}$, $J(H, H)=2.4 \mathrm{~Hz}, 1 \mathrm{H} ; \mathrm{ArH}), 7.37$ (m, $1 \mathrm{H} ; \mathrm{ArH}), 7.30$ (m, $2 \mathrm{H} ; \mathrm{ArH})$;

${ }^{13} \mathrm{C} \mathrm{NMR}\left(\mathrm{CDCl}_{3}, 100 \mathrm{MHz}\right) \delta: 135.1,129.0,128.6,123.3,120.9,119.9,111.6,97.5,78.8\left(\mathrm{t}, J^{l}(C, D)=\right.$ $38.2 \mathrm{~Hz}, \mathrm{C} \equiv C \mathrm{CD}), 77.2\left(\mathrm{t}, J^{2}(C, D)=7.7 \mathrm{~Hz}, C \equiv \mathrm{CD}\right)$.

\section{Mechanistic Investigations}

\section{Preparation and reaction of ${ }^{13} \mathrm{C}$-labeled reagent $1 \mathrm{~d}$ '}

2-(4-Methoxybenzyloxy)ethanol (17)

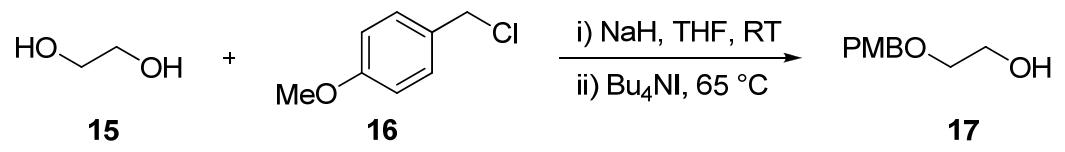

Following a reported procedure ${ }^{[8]}$ sodium hydride $(60 \%$ in mineral oil, $0.70 \mathrm{~g}, 17 \mathrm{mmol}, 1.0$ equiv) was added to a solution of ethylene glycol (15) (freshly distilled from drierite ( $\left.\mathrm{p}=0.3 \mathrm{mbar}, \mathrm{T}=46^{\circ} \mathrm{C}\right), 2.8 \mathrm{~mL}$, $50 \mathrm{mmol}, 3.0$ equiv) in THF (30 mL). After stirring $30 \mathrm{~min}$ at RT, 4-methoxybenzyl chloride (16) $(2.60 \mathrm{~g}$, $16.6 \mathrm{mmol}, 1.00$ equiv) and $\mathrm{Bu}_{4} \mathrm{NI}(0.61 \mathrm{~g}, 1.7 \mathrm{mmol}, 0.10$ equiv) were added, and the reaction mixture was heated to reflux. After $4.5 \mathrm{~h}$, the reaction mixture was cooled to RT, the reaction was quenched with sat. $\mathrm{NH}_{4} \mathrm{Cl}(30 \mathrm{~mL})$ and extracted with $\mathrm{Et}_{2} \mathrm{O}(3 \times 30 \mathrm{~mL})$. The combined organic layers were washed with brine $(30 \mathrm{~mL})$, dried over $\mathrm{MgSO}_{4}$ and the solvent was removed under reduced pressure. The crude product was purified by flash column chromatography (PET/AcOEt 5/1-1/1) to yield protected alcohol 17 (2.54 g, 13.9 mmol, $84 \%$ ) as a yellow oil.

$R_{f}\left(\mathrm{PET} / \mathrm{AcOEt} 1 / 1, \mathrm{KMnO}_{4}\right)$ 0.25;

${ }^{1} \mathrm{H}$ NMR $\left(\mathrm{CDCl}_{3}, 400 \mathrm{MHz}\right) \delta 7.27(\mathrm{dm}, J(H, H)=8.2 \mathrm{~Hz}, 2 \mathrm{H} ; \mathrm{ArH}), 6.89(\mathrm{dm}, J(H, H)=8.6 \mathrm{~Hz}, 2 \mathrm{H}$; ArH), 4.49 (s, $2 \mathrm{H}$; benzyl CH$), 3.81\left(\mathrm{~s}, 3 \mathrm{H} ; \mathrm{OCH}_{3}\right), 3.74$ (t, $J(H, H)=4.3 \mathrm{~Hz}, 2 \mathrm{H}$; $\left.\mathrm{CH}_{2} \mathrm{OPMB}\right), 3.57$ (m, 2 $\mathrm{H} ; \mathrm{CH}_{2} \mathrm{OH}$ ), 2.08 (br s, $1 \mathrm{H} ; \mathrm{OH}$ ).

${ }^{1} \mathrm{H}$ NMR corresponded to the literature values. ${ }^{[8]}$

2-(4-Methoxybenzyloxy)acetaldehyde (20)

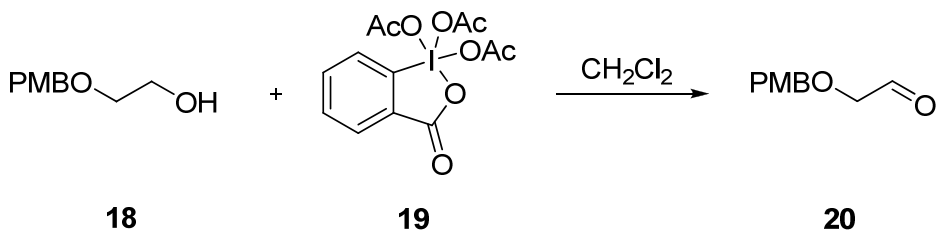

[8] K. Masutani, T. Minowa, Y. Hagiwara, T. Mukaiyama, Bull. Chem. Soc. Jpn. 2006, 79, 1106. 
Following a reported procedure, ${ }^{[9]}$ Dess-Martin Periodinane (19) $(0.53 \mathrm{~g}, 1.3 \mathrm{mmol}, 1.1$ equiv) was added to a solution of alcohol $18\left(0.21 \mathrm{~g}, 1.2 \mathrm{mmol}, 1.0\right.$ equiv) in wet $\mathrm{CH}_{2} \mathrm{Cl}_{2}(9 \mathrm{~mL})$. After stirring $2.5 \mathrm{~h}$ at RT, the reaction was quenched with sat. $\mathrm{NaHCO}_{3}(10 \mathrm{~mL})$ and sat. sodium thiosulfate solution $(10 \mathrm{~mL})$ and the mixture was stirred vigorously for $10 \mathrm{~min}$ until two clear layers were obtained. The layers were separated and the water layer was extracted with $\mathrm{CH}_{2} \mathrm{Cl}_{2}(3 \times 20 \mathrm{~mL})$. The combined organic layers were dried over $\mathrm{MgSO}_{4}$ and the solvent was removed under reduced pressure to give aldehyde $20(0.21 \mathrm{~g}, 1.2$ mmol, quant.) as a yellow oil, which was used immediately in the next step without further purification.

$R_{f}\left(\mathrm{PET} / \mathrm{AcOEt} 1 / 1, \mathrm{KMnO}_{4}\right)$ 0.35;

${ }^{1} \mathrm{H}$ NMR $\left(\mathrm{CDCl}_{3}, 400 \mathrm{MHz}\right) \delta 9.71(\mathrm{t}, J(H, H)=0.9 \mathrm{~Hz}, \mathrm{CHO}), 7.29(\mathrm{dm}, J(H, H)=8.8 \mathrm{~Hz}, 2 \mathrm{H} ; \mathrm{ArH})$, $6.90\left(\mathrm{dm}, J(H, H)=8.8 \mathrm{~Hz}, 2 \mathrm{H}\right.$; ArH), $4.57(\mathrm{~s}, 2 \mathrm{H}$; benzyl CH$), 4.07\left(\mathrm{~d}, J(H, H)=0.9 \mathrm{~Hz}, 2 \mathrm{H} ; \mathrm{CH}_{2} \mathrm{CHO}\right)$, $3.81\left(\mathrm{~s}, 3 \mathrm{H} ; \mathrm{OCH}_{3}\right)$.

${ }^{1} \mathrm{H}$ NMR corresponded to the literature values. ${ }^{[9]}$

Labeled 1-((3,3-dibromoallyloxy)methyl)-4-methoxybenzene (23) and triiso-propyl(3-(4methoxybenzyloxy)prop-1-ynyl)silane (24)

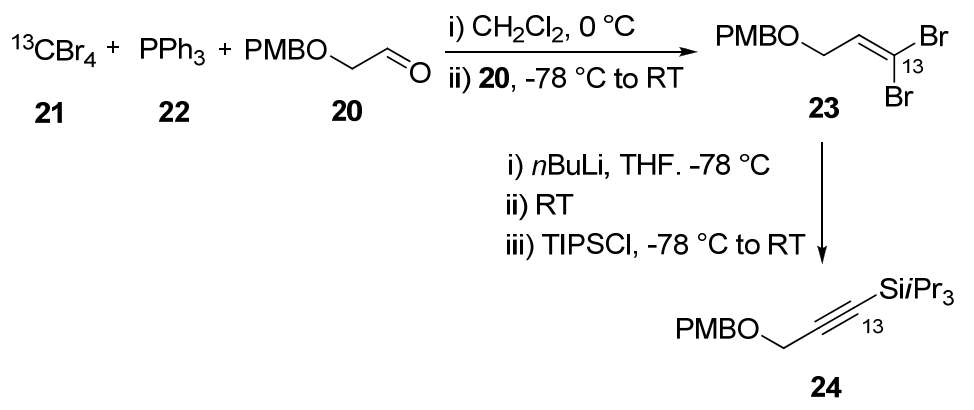

Following a slightly modified literature procedure, ${ }^{[10]}$ a solution of $\mathrm{PPh}_{3}$ (22) (1.6 g, $6.0 \mathrm{mmol}, 2.0$ equiv) in $\mathrm{CH}_{2} \mathrm{Cl}_{2}(9 \mathrm{~mL})$ was added to a solution of $\mathrm{CBr}_{4}(\mathbf{2 1})\left(1.0 \mathrm{~g}, 3.0 \mathrm{mmol}, 1.0\right.$ equiv, $20 \%{ }^{13} \mathrm{C}$, prepared from $0.80 \mathrm{~g}$ natural $\mathrm{CBr}_{4}$ and $0.20 \mathrm{~g} 99 \%{ }^{13} \mathrm{C}$-enriched $\left.\mathrm{CBr}_{4}\right)$ in $\mathrm{CH}_{2} \mathrm{Cl}_{2}(12 \mathrm{~mL})$ at $0{ }^{\circ} \mathrm{C}$ over 15 min. After stirring for $15 \mathrm{~min}$ at $0{ }^{\circ} \mathrm{C}$, the yellow-orange solution was cooled to $-78{ }^{\circ} \mathrm{C}$ and a solution of aldehyde 20 (freshly synthesized, $0.66 \mathrm{~g}, 3.6 \mathrm{mmol}, 1.2$ equiv) in $\mathrm{CH}_{2} \mathrm{Cl}_{2}(9 \mathrm{~mL}$ ) was added over $10 \mathrm{~min}$, whereas the reaction mixture turned dark red-brown. The reaction mixture was left to warm to RT over $17 \mathrm{~h}$, quenched with sat. $\mathrm{NaHCO}_{3}(30 \mathrm{~mL})$ and extracted with $\mathrm{CH}_{2} \mathrm{Cl}_{2}(3 \times 30 \mathrm{~mL})$. The combined organic layers were washed with brine $(30 \mathrm{~mL})$, dried over $\mathrm{MgSO}_{4}$ and the solvent was removed under reduced pressure. The crude product was purified by flash column chromatography $\left(\mathrm{PET} / \mathrm{CH}_{2} \mathrm{Cl}_{2} 3 / 1-1.5 / 1\right)$ to yield dibromide $23(501 \mathrm{~g}, 1.55 \mathrm{mmol}, 52 \%)$ as a slightly yellow oil, which was used directly in the next step.

$R_{f}\left(\mathrm{PET} / \mathrm{CH}_{2} \mathrm{Cl}_{2} 2 / 1, \mathrm{KMnO}_{4}\right)$ 0.30;

${ }^{1} \mathrm{H}$ NMR $\left(\mathrm{CDCl}_{3}, 400 \mathrm{MHz}\right) \delta 7.28(\mathrm{dm}, J(H, H)=8.6 \mathrm{~Hz}, 2 \mathrm{H} ; \mathrm{ArH}), 6.90(\mathrm{dm}, J(H, H)=8.6 \mathrm{~Hz}, 2 \mathrm{H}$; ArH), $6.64(\mathrm{tm}, J(H, H)=6.1 \mathrm{~Hz}, 1 \mathrm{H}$; alkene $\mathrm{H}), 4.46\left(\mathrm{~s}, 2 \mathrm{H}\right.$; benzyl $\left.\mathrm{CH}_{2}\right), 4.04\left(\mathrm{~m}, 2 \mathrm{H}\right.$; alkene $\left.\mathrm{CH}_{2}\right), 3.81$ $\left(\mathrm{s}, 3 \mathrm{H} ; \mathrm{OCH}_{3}\right)$;

[9] I. Collins, J. Caldwell, T. Fonseca, A. Donald, V. Bavetsias, L. J. K. Hunter, M. D. Garrett, M. G. Rowlands, G. W. Aherne, T. G. Davies, V. Berdini, S. J. Woodhead, D. Davis, L. C. A. Seavers, P. G. Wyatt, P. Workman, E. McDonald, Bioorg. Med. Chem. 2006, 14, 1255.

[10] L. A. Paquette, J. Y. Chang, Z. S. Liu, J. Org. Chem. 2004, 69, 6441. 
${ }^{13} \mathrm{C} \mathrm{NMR}\left(\mathrm{CDCl}_{3}, 100 \mathrm{MHz}\right) \delta 159.3,135.6,129.5,129.4,113.8,91.3$ (labeled 20x more intensive), $72.2,69.4,55.2$

Following a literature procedure ${ }^{[11]} n \operatorname{BuLi}(2.5 \mathrm{M}$ in hexane, $1.4 \mathrm{~mL}, 3.4 \mathrm{mmol}, 2.2$ equiv) was added dropwise to a solution of dibromide $23\left(0.50 \mathrm{~g}, 1.5 \mathrm{mmol}, 1.0\right.$ equiv) in THF $(9 \mathrm{~mL})$ at $-78^{\circ} \mathrm{C}$. The yellow solution was stirred $1 \mathrm{~h}$ at $-78{ }^{\circ} \mathrm{C}$ and $1 \mathrm{~h}$ at RT. After cooling to $-78{ }^{\circ} \mathrm{C}$, TIPSCl $(0.43 \mathrm{~mL}, 2.0 \mathrm{mmol}, 1.3$ equiv) was added and the reaction was left to warm to RT over $12 \mathrm{~h}$. The reaction was quenched with sat. $\mathrm{NaHCO}_{3}(10 \mathrm{~mL})$ and extracted with $\mathrm{Et}_{2} \mathrm{O}(3 \times 20 \mathrm{~mL})$. The combined organic layers were washed with brine $(10 \mathrm{~mL})$, dried over $\mathrm{MgSO}_{4}$ and the solvent was removed under reduced pressure. The crude product was purified by flash column chromatography $\left(\mathrm{PET} / \mathrm{CH}_{2} \mathrm{Cl}_{2} 4 / 1-2 / 1\right)$ to yield alkyne 24 (372 g, $1.12 \mathrm{mmol}$, $72 \%$ ) as a colorless oil. Comparison of the ${ }^{13} \mathrm{C}$ NMR with an unlabeled sample (synthesized following the same procedure) showed $20 \%{ }^{13} \mathrm{C}$ incorporation at the indicated position only.

$R_{f}\left(\mathrm{PET} / \mathrm{CH}_{2} \mathrm{Cl}_{2} 2 / 1, \mathrm{KMnO}_{4}\right)$ 0.35;

${ }^{1} \mathrm{H}$ NMR $\left(\mathrm{CDCl}_{3}, 400 \mathrm{MHz}\right) \delta 7.30(\mathrm{dm}, J(H, H)=8.4 \mathrm{~Hz}, 2 \mathrm{H} ; \mathrm{ArH}), 6.89(\mathrm{dm}, J(H, H)=8.6 \mathrm{~Hz}, 2 \mathrm{H}$; ArH), 4.59 (s, $2 \mathrm{H}$; benzyl CH 2$), 4.19$ (s, $2 \mathrm{H}$; alkyne $\mathrm{CH}_{2}$ ), 3.81 (s, $\left.3 \mathrm{H} ; \mathrm{OCH}_{3}\right), 1.12$ (m, $21 \mathrm{H}$; TIPS);

${ }^{13} \mathrm{C}$ NMR $\left(\mathrm{CDCl}_{3}, 100 \mathrm{MHz}\right) \delta 159.3,129.8,129.5,113.8,103.4,87.7$ (labeled 20x more intensive), 70.6, 57.4, 55.2, 18.6, 11.1;

IR v $2961(\mathrm{w}), 2944(\mathrm{w}), 2931(\mathrm{w}), 2866(\mathrm{w}), 2171(\mathrm{w}), 1663(\mathrm{w}), 1614(\mathrm{w}), 1515(\mathrm{w}), 1463(\mathrm{w}), 1444$ (w), $1378(\mathrm{w}), 1250(\mathrm{w}), 1078(\mathrm{~m}), 1036(\mathrm{w}), 907$ (s), $730(\mathrm{~s}), 651(\mathrm{~m})$;

HRMS(ESI) calcd for $\mathrm{C}_{15} \mathrm{H}_{16} \mathrm{O}_{3}{ }^{+}(\mathrm{M}+\mathrm{H}) 333.2250$, found 333.2254.

Isotope repartition: expected for about $20 \%$ incorporation was obtained.

\section{Labeled triiso-propyl acetylene (12')}

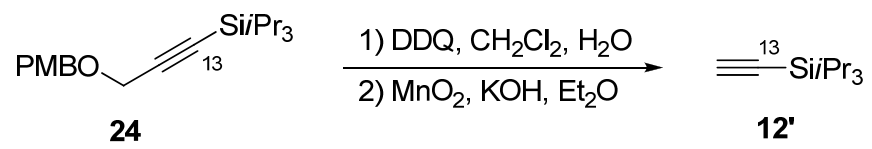

Following a literature procedure ${ }^{[12]} \mathrm{DDQ}(0.38 \mathrm{~g}, 1.7 \mathrm{mmol}, 1.5$ equiv) was added to a solution of protected alcohol 24 (372 mg, $1.12 \mathrm{mmol}, 1.00$ equiv) in $\mathrm{CH}_{2} \mathrm{Cl}_{2}(11 \mathrm{~mL})$ and water $(1.1 \mathrm{~mL})$ at $0{ }^{\circ} \mathrm{C}$. The reaction mixture was stirred $15 \mathrm{~min}$ at $0{ }^{\circ} \mathrm{C}$ and $3 \mathrm{~h}$ at RT. The resulting dark red thick suspension was quenched with sat. $\mathrm{NaHCO}_{3}(20 \mathrm{~mL})$ and extracted with $\mathrm{CH}_{2} \mathrm{Cl}_{2}(3 \times 20 \mathrm{~mL})$. The combined organic layers were washed with sat. $\mathrm{NaHCO}_{3}(20 \mathrm{~mL})$ and brine $(20 \mathrm{~mL})$, dried over $\mathrm{MgSO}_{4}$ and the solvent was removed under reduced pressure. TLC (PET/AcOEt 6/1, $\left.\mathrm{KMnO}_{4}\right)$ showed a mixture of two products, which were shown to be the corresponding propargylic alcohol $\left(R_{f}=0.50\right)$ and anisaldehyde $\left(R_{f}=0.45\right)$ by ${ }^{1} H$ NMR. This mixture was directly used as such in the next step.

Following a literature procedure, ${ }^{[13]}$ the obtained mixture was diluted in $\mathrm{Et}_{2} \mathrm{O}(14 \mathrm{~mL})$ and $\mathrm{MnO}_{2}$ (Aldrich activated, $1.2 \mathrm{~g}, 13 \mathrm{mmol}, 12$ equiv) and $\mathrm{KOH}$ (freshly grounded, $0.38 \mathrm{~g}, 6.8 \mathrm{mmol}, 6.0$ equiv) were added in 4 portions every hour. After stirring for further $3 \mathrm{~h}$, TLC (PET/AcOEt 6/1, $\mathrm{KMnO}_{4}$ ) showed

[11] E. J. Corey, P. L Fuchs,. Tetrahedron Lett. 1972, 3769.

[12] P. Dimopoulos, A. Athlan, S. Manaviazar, J. George, M. Walters, L. Lazarides, A. E. Aliev, K. J. Hale, Org. Lett. 2005, 7, 5369.

[13] H. Kukula, S. Veit, A. Godt, Eur. J. Org. Chem. 1999, 277. 
complete conversion and the reaction mixture was filtered over $\mathrm{SiO}_{2}$ and the filter cake was washed with $\mathrm{Et}_{2} \mathrm{O}(50 \mathrm{~mL})$. The solvent was removed under reduced pressure and the crude mixture was purified by flash column chromatography (PET) to yield alkyne 12' (167 mg, $0.915 \mathrm{mmol}, 82 \%)$ as a colorless oil. Comparison of the ${ }^{13} \mathrm{C}$ NMR with an unlabeled sample (synthesized following the same procedure) showed $20 \%{ }^{13} \mathrm{C}$ incorporation at the indicated position only.

$R_{f}\left(\mathrm{PET}, \mathrm{KMnO}_{4}\right)$ 0.80;

${ }^{1} \mathrm{H}$ NMR $\left(\mathrm{CD}_{2} \mathrm{Cl}_{2}, 400 \mathrm{MHz}\right) \delta 2.38$ (s, $1 \mathrm{H}$; alkyne H), 1.07 (m, $21 \mathrm{H}$; TIPS);

${ }^{13} \mathrm{C}$ NMR $\left(\mathrm{CD}_{2} \mathrm{Cl}_{2}, 100 \mathrm{MHz}\right) \delta 94.9,86.2$ (labeled 20x more intensive), 18.3, 11.1.

Labeled triiso-propyl((trimethylsilyl)ethynyl)silane (11’)

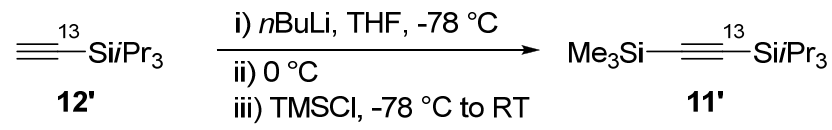

$n \operatorname{BuLi}(2.5 \mathrm{M}$ in hexane, $0.44 \mathrm{~mL}, 1.1 \mathrm{mmol}, 1.2$ equiv) was added to a solution of acetylene 12' (167 $\mathrm{mg}, 0.915 \mathrm{mmol}, 1.00$ equiv) in $\operatorname{THF}(2 \mathrm{~mL})$ at $-78{ }^{\circ} \mathrm{C}$. The reaction mixture was stirred $15 \mathrm{~min}$ at $0{ }^{\circ} \mathrm{C}$ and the yellow solution was cooled back to $-78^{\circ} \mathrm{C}$. $\mathrm{TMSCl}$ (freshly distilled, $0.15 \mathrm{~mL}, 1.2 \mathrm{mmol}, 1.3$ equiv) was added and the colorless solution was left to warm to RT over $6 \mathrm{~h}$. The reaction was quenched with sat. $\mathrm{NH}_{4} \mathrm{Cl}(3 \mathrm{~mL})$ and extracted with $\mathrm{Et}_{2} \mathrm{O}(3 \times 10 \mathrm{~mL})$. The combined organic layers were washed with brine $(5 \mathrm{~mL})$, dried over $\mathrm{MgSO}_{4}$ and the solvent was removed under reduced pressure. The crude mixture was purified by flash column chromatography (PET) to yield protected alkyne 11' (184 g, $0.722 \mathrm{mmol}, 79 \%)$ as a colorless oil. Comparison of the ${ }^{13} \mathrm{C}$ NMR with an unlabeled sample showed $20 \%{ }^{13} \mathrm{C}$ incorporation at the indicated position only.

$R_{f}\left(\mathrm{PET}, \mathrm{KMnO}_{4}\right)$ 0.80;

${ }^{1} \mathrm{H}$ NMR $\left(\mathrm{CDCl}_{3}, 400 \mathrm{MHz}\right) \delta 1.07$ (m, $21 \mathrm{H}$; TIPS), 0.17 (s, $9 \mathrm{H}$; TMS);

${ }^{13} \mathrm{C} \mathrm{NMR}\left(\mathrm{CDCl}_{3}, 100 \mathrm{MHz}\right) \delta 116.2,110.1$ (labeled 20x more intensive), 18.6, 11.1, 0.0;

IR v $2959(\mathrm{~m}), 2944(\mathrm{~m}), 2896(\mathrm{w}), 2867(\mathrm{~m}), 1464(\mathrm{w}), 1385(\mathrm{w}), 1250(\mathrm{~m}), 996(\mathrm{w}), 842(\mathrm{~s}), 764(\mathrm{~s})$, $675(\mathrm{~m}), 660(\mathrm{~m})$.

${ }^{1} \mathrm{H}$ NMR corresponded to the literature values. ${ }^{[4]}$

Labeled 1-[(triiso--propyllsilyl)ethynyl]-1,2-benziodoxol-3(1H)-one (1d')

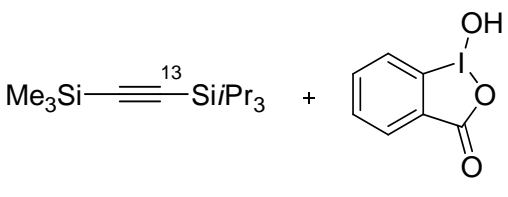

$11^{\prime}$

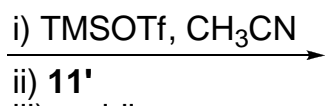

iii) pyridine

9

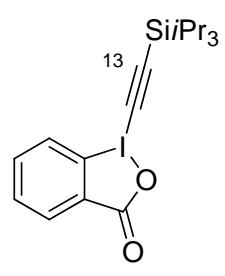

$1 d^{\prime}$

Following a slightly modified literature procedure, ${ }^{3}$ TMSOTf (freshly distilled, $0.15 \mathrm{~mL}, 0.82 \mathrm{mmol}, 1.1$ equiv) was added to a suspension of iodinane 9 (freshly synthesized, $0.19 \mathrm{~g}, 0.72 \mathrm{mmol}, 1.0$ equiv) in $\mathrm{CH}_{3} \mathrm{CN}(6.5 \mathrm{~mL})$. After $10 \mathrm{~min}$, a solution of acetylene 11' $\left(0.18 \mathrm{~g}, 0.72 \mathrm{mmol}, 1.0\right.$ equiv) in $\mathrm{CH}_{2} \mathrm{Cl}_{2}(1.0$ 
$\mathrm{mL}$ ) was added to the slightly yellow solution. After stirring $15 \mathrm{~min}$ at RT, pyridine $(70 \mu \mathrm{L}, 0.87 \mathrm{mmol}, 1.2$ equiv) was added and the solvent was removed under reduced pressure below $30^{\circ} \mathrm{C}$. The reaction mixture was diluted with $\mathrm{CH}_{2} \mathrm{Cl}_{2}(15 \mathrm{~mL})$ and washed with $1 \mathrm{M} \mathrm{HCl}(5 \mathrm{~mL})$. The water layer was extracted with $\mathrm{CH}_{2} \mathrm{Cl}_{2}(2 \times 5 \mathrm{~mL})$ and the combined organic layers were washed with sat. $\mathrm{Na}_{2} \mathrm{CO}_{3}(2 \times 10 \mathrm{~mL})$. The combined basic aqueous layers were extracted with $\mathrm{CH}_{2} \mathrm{Cl}_{2}(10 \mathrm{~mL})$ and the combined organic layers were dried over $\mathrm{MgSO}_{4}$ and the solvent was removed under reduced pressure to give iodinane 1d' ( $>95 \%$ pure by ${ }^{1} \mathrm{HNMR}$, containing traces of acetylene 11', $259 \mathrm{mg}, 0.604 \mathrm{mmol}, 84 \%$ ) as a slightly yellow solid. Comparison of the ${ }^{13} \mathrm{C}$ NMR with an unlabeled sample showed $20 \%{ }^{13} \mathrm{C}$ incorporation at the indicated position only.

${ }^{1} \mathrm{H}$ NMR $\left(\mathrm{CDCl}_{3}, 400 \mathrm{MHz}\right) \delta 8.40(\mathrm{~m}, 1 \mathrm{H} ; \mathrm{Ar} \mathrm{H}), 8.28(\mathrm{~m}, 1 \mathrm{H} ; \mathrm{Ar} \mathrm{H}), 7.74(\mathrm{~m}, 2 \mathrm{H} ; \mathrm{Ar} \mathrm{H}), 1.13(\mathrm{~m}$, $21 \mathrm{H}$; TIPS);

${ }^{13} \mathrm{C} \mathrm{NMR}\left(\mathrm{CDCl}_{3}, 100 \mathrm{MHz}\right) \delta 166.3,134.6,132.4,131.5,131.4,126.0,115.6,114.1$ (labeled 20x more intensive), 64.7, 18.4, 11.1 .

\section{Labeled 3-((Triiso-propylsilyl)ethynyl)-1H-indole (3a')}

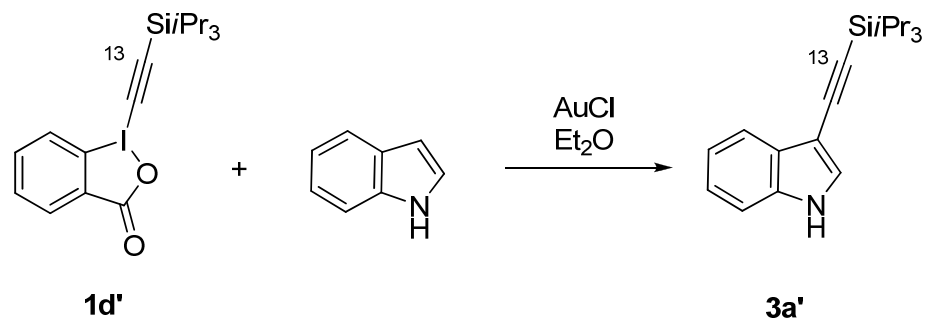

Following the general procedure on a $0.20 \mathrm{mmol}$ scale using 1 d' $\left(103 \mathrm{mg}, 0.240 \mathrm{mmol}, 10 \%{ }^{13} \mathrm{C}\right.$, prepared from $51.5 \mathrm{mg} 20 \%$ labeled 1-[(triiso-propyllsilyl)ethynyl]-1,2-benziodoxol-3(1H)-one and 51.5 $\mathrm{mg}$ natural 1d). Purification by flash chromatography (PET/Et ${ }_{2} \mathrm{O}$ 8/2) afforded 3a' (51 mg, $0.17 \mathrm{mmol}$, $86 \%)$ as a brown solid.

$\mathrm{R} f 0.4\left(\mathrm{PET} / \mathrm{Et}_{2} \mathrm{O} 7 / 3, \mathrm{UV} /\right.$ Anisaldehyde);

${ }^{1} \mathrm{H}$ NMR $\left(\mathrm{CDCl}_{3}, 400 \mathrm{MHz}\right) \delta 8.11($ br s, $1 \mathrm{H} ; \mathrm{NH}), 7.79(\mathrm{~m}, 1 \mathrm{H} ; \mathrm{ArH}), 7.41(\mathrm{~d}, J(H, H)=2.6 \mathrm{~Hz}, 1 \mathrm{H}$; ArH), 7.36 (m, $1 \mathrm{H} ; \mathrm{ArH}), 7.26$ (m, 2H; ArH), 1.22 (m, $21 \mathrm{H}$; TIPS);

${ }^{13} \mathrm{C} \mathrm{NMR}\left(\mathrm{CDCl}_{3}, 100 \mathrm{MHz}\right) \delta 135.1,128.9,128.2,123.1,120.8,120.1,111.4,100.3,99.2,92.9$ (labeled 10x more intensive), 18.8, 11.5;

Comparison of the ${ }^{13} \mathrm{C}$ NMR with an unlabeled sample showed $10 \%{ }^{13} \mathrm{C}$ incorporation at the indicated position only.

\section{Labeled 3-Ethynyl-1H-indole 14'}
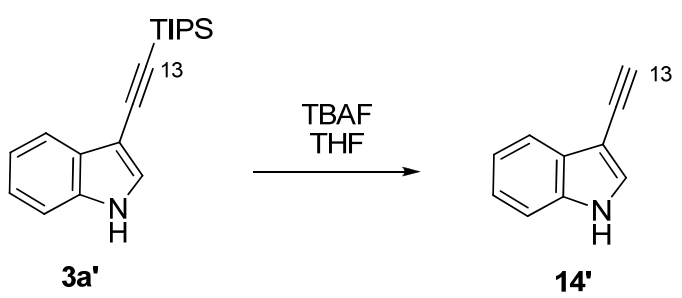
Tetrabutylammonium fluoride ( $1 \mathrm{M}$ in THF, $0.34 \mathrm{~mL}, 0.34 \mathrm{mmol}, 2.0$ equiv) was added to a stirring solution of 3b' ( $50 \mathrm{mg}, 0.17 \mathrm{mmol}, 1$ equiv) in THF $(2.5 \mathrm{~mL})$. The reaction was stirred at room temperature for $15 \mathrm{~h}$. The reaction was quenched with a saturated solution of $\mathrm{NH}_{4} \mathrm{Cl}(5 \mathrm{~mL})$ and extracted twice with $\mathrm{Et}_{2} \mathrm{O}(10 \mathrm{~mL})$. The organic layers were combined, washed with saturated $\mathrm{NaHCO}_{3}(10 \mathrm{~mL})$, brine $(10 \mathrm{~mL})$, dried with $\mathrm{MgSO}_{4}$ and concentrated under reduced pressure. The crude mixture was purified by flash chromatography (PET/Et ${ }_{2} \mathrm{O}$ 9/1 to 9/2) to afford $14(17 \mathrm{mg}, 0.12 \mathrm{mmol}, 71 \%)$ as a tan solid.

$\mathrm{R} f 0.2\left(\mathrm{PET} / \mathrm{Et}_{2} \mathrm{O} 9 / 1, \mathrm{UV} / \mathrm{KMnO}_{4}\right)$

${ }^{1} \mathrm{H}$ NMR $\left(\mathrm{CDCl}_{3}, 400 \mathrm{MHz}\right) \delta: 8.19$ (br s, $\left.1 \mathrm{H} ; \mathrm{NH}\right), 7.80(\mathrm{dd}, J(H, H)=0.5,7.4 \mathrm{~Hz}, 1 \mathrm{H} ; \mathrm{ArH}), 7.47$ (dd, $J(H, H)=1.0,2.4 \mathrm{~Hz}, \mathrm{ArH}) 7.40(\mathrm{~m}, 1 \mathrm{H}$; ArH), $7.27(\mathrm{~m}, 2 \mathrm{H}$; ArH), $3.26(\mathrm{~d}, J(H, H)=0.7 \mathrm{~Hz}, 1 \mathrm{H}$; acetylene $\mathrm{H})$;

${ }^{13} \mathrm{C} \mathrm{NMR}\left(\mathrm{CDCl}_{3}, 100 \mathrm{MHz}\right) \delta: 135.0,128.8,128.5,123.2,120.9,120.0,111.4,97.7,78.9$ (labeled 10x more intensive), 77.5;

Comparison of the ${ }^{13} \mathrm{C}$ NMR with an unlabeled sample showed $10 \%{ }^{13} \mathrm{C}$ incorporation at the indicated position only.

\section{Spectra of New Compounds}


solvent: $<\mathrm{CDCI} 3>$

Frequency.400.13 $\mathrm{MHz}$

$\overbrace{\mathrm{TfO}^{-}}^{\mathrm{SiiPr}_{3}}$

$1 b$

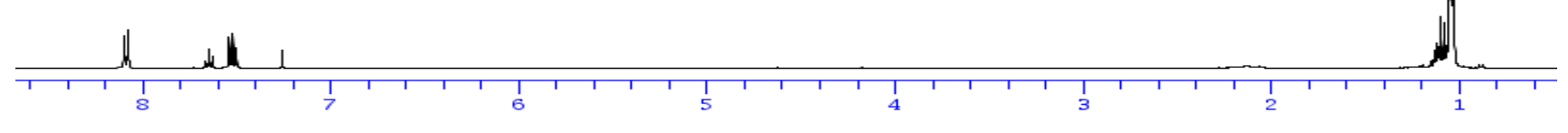

solvent: $<\mathrm{CDCl} 3>$

$61 \mathrm{MHz}$
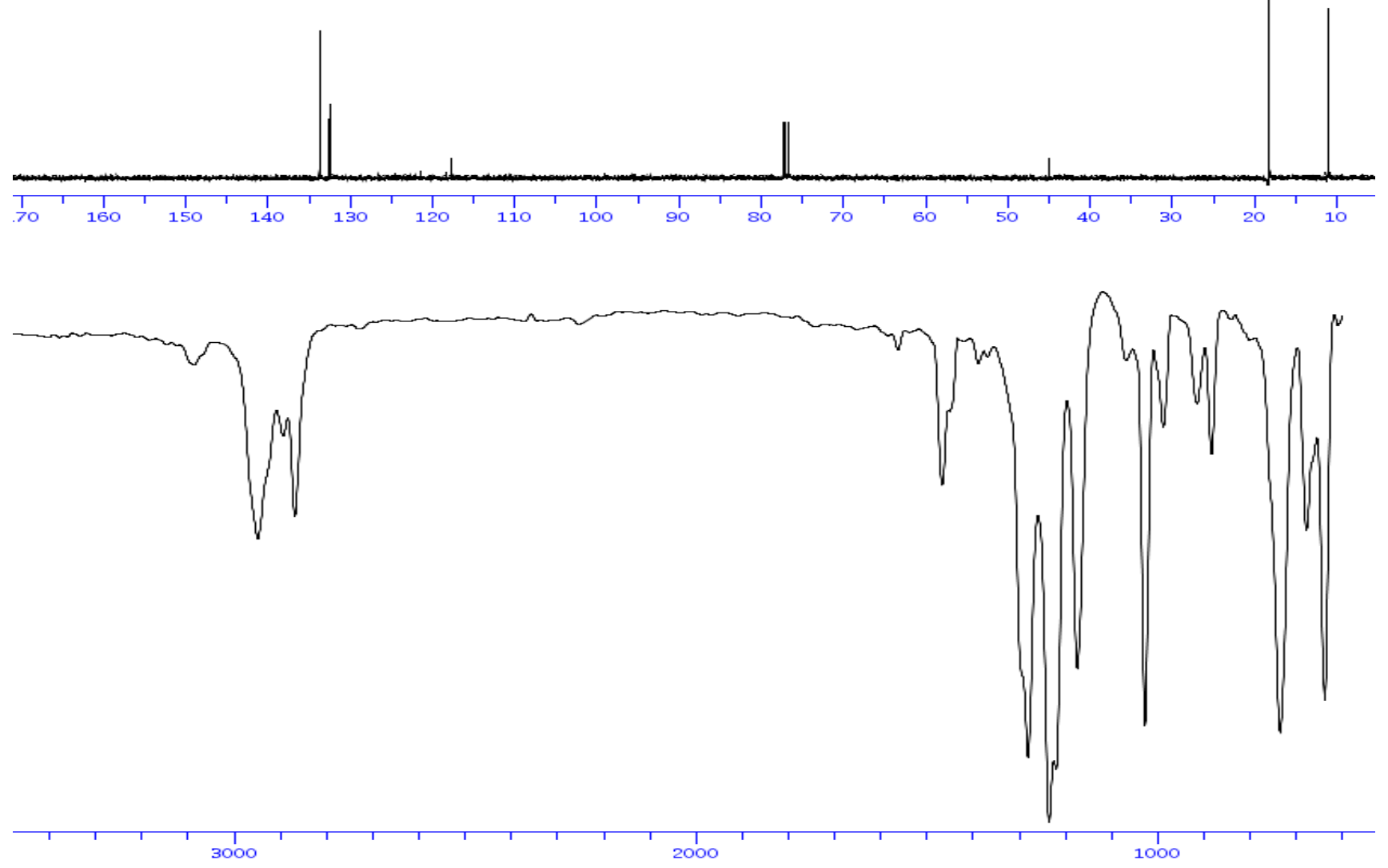
solvent: $<\mathrm{CDCl} 3>$
Frequency $400.13 \mathrm{MHz}$
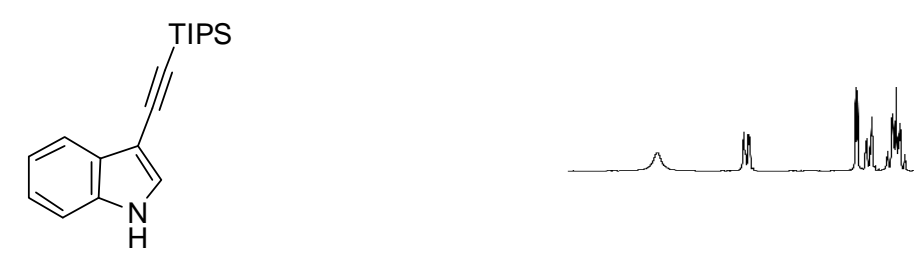

$3 a$

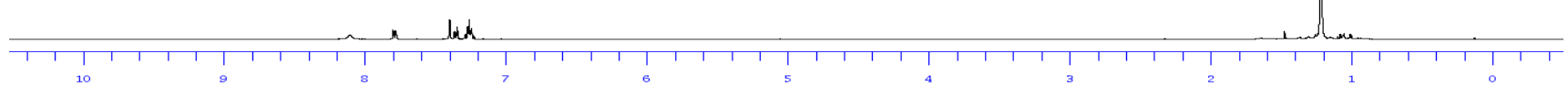

solvent: $\langle\mathrm{CDCI} 3\rangle$

Frequency. $100.61 \mathrm{MHz}$
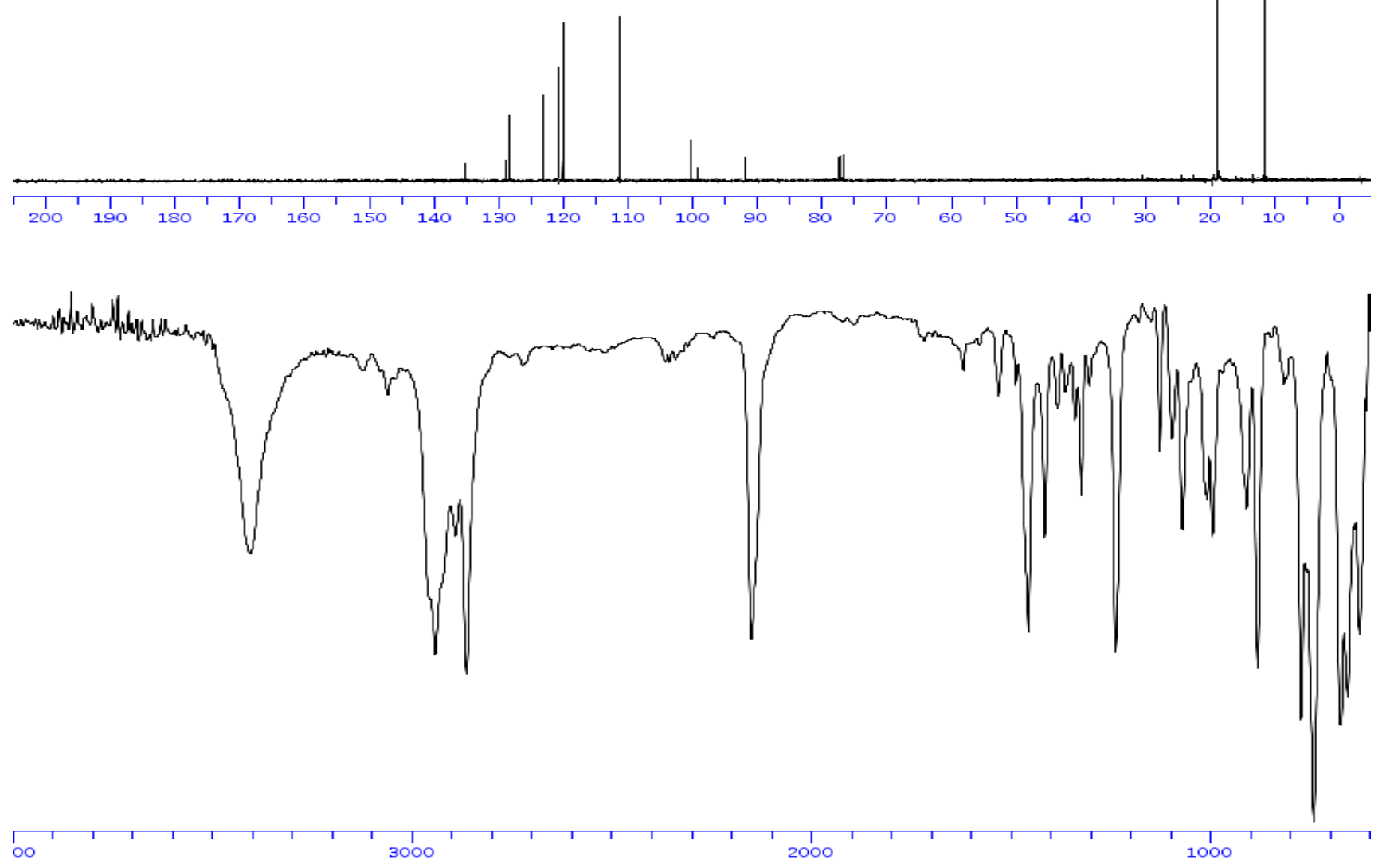
solvent: $<C D C I 3>$
Frequency. $400.13 \mathrm{MHz}$
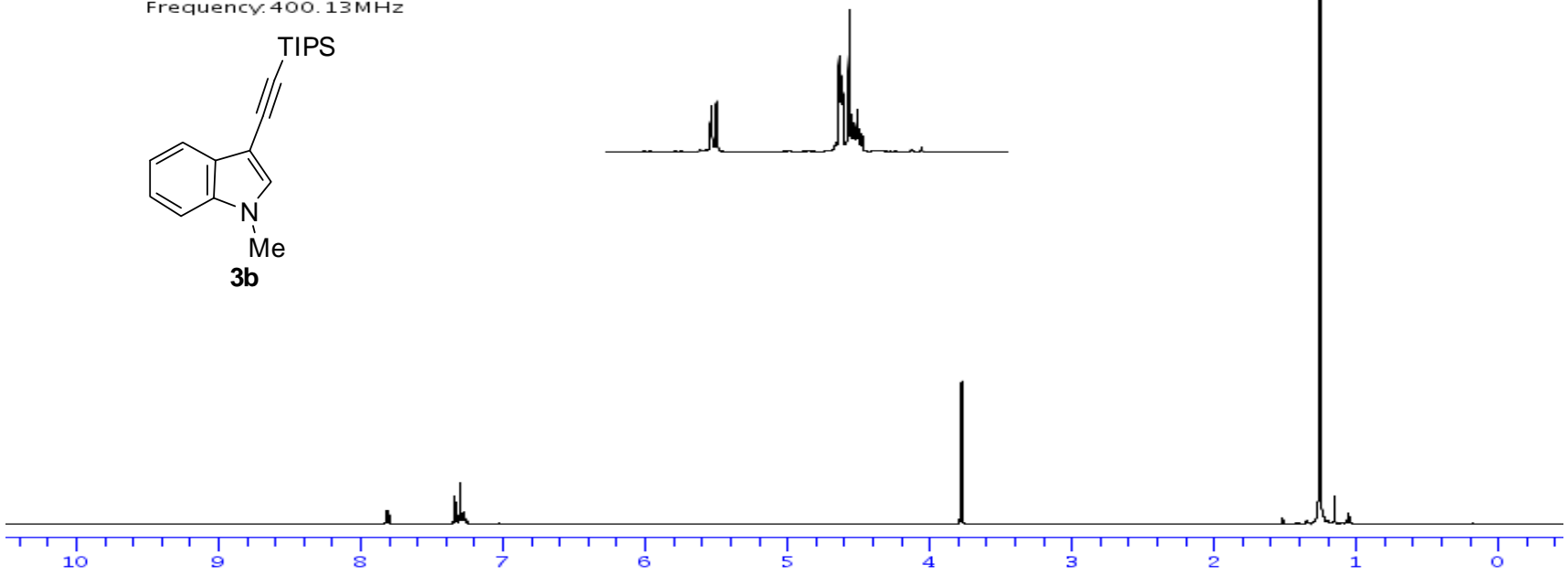

solvent: $\angle C D C I 3>$
Frequency. $100.61 \mathrm{MHz}$
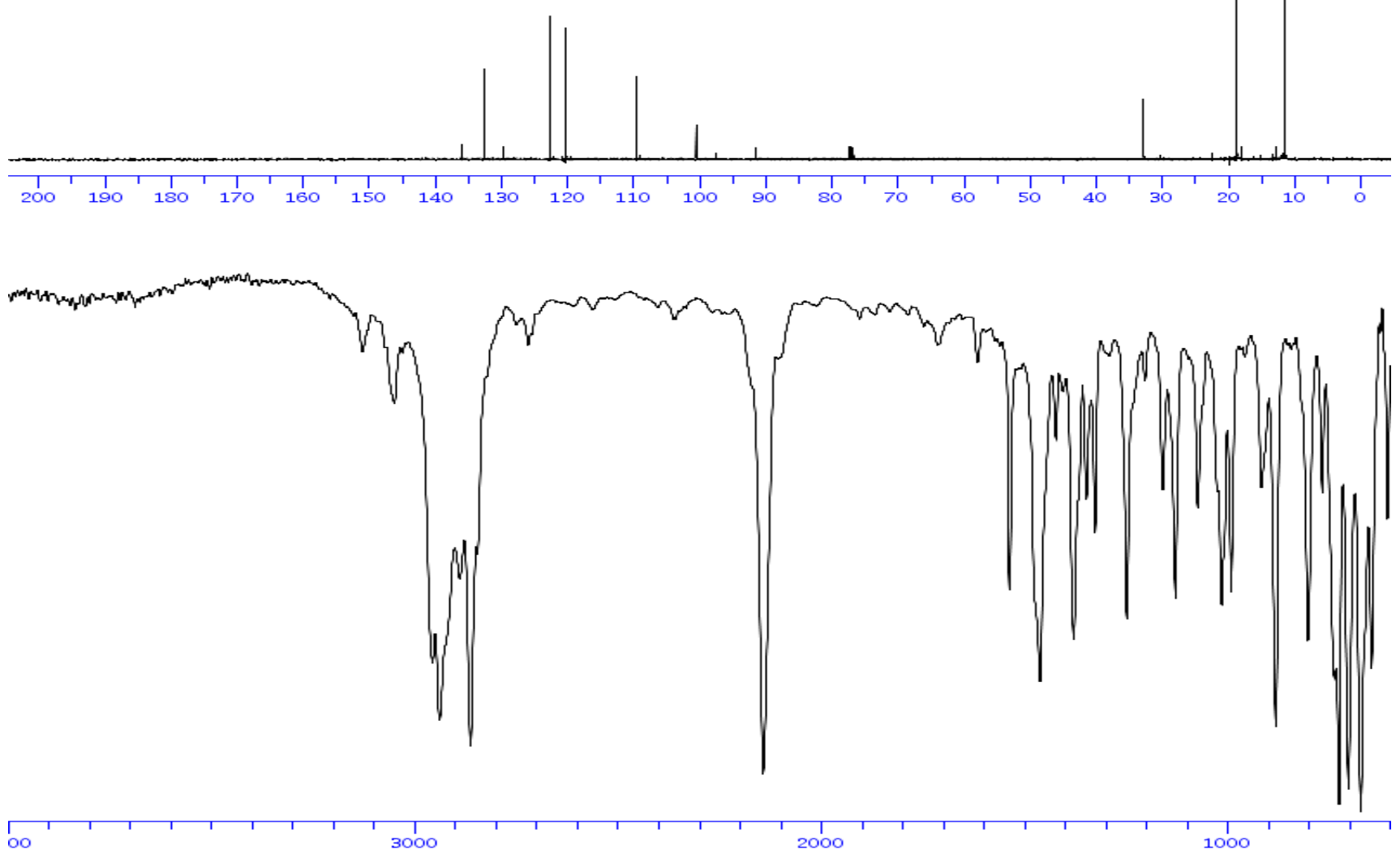

S29 
Solvent: $<\mathrm{CDCl} 3$

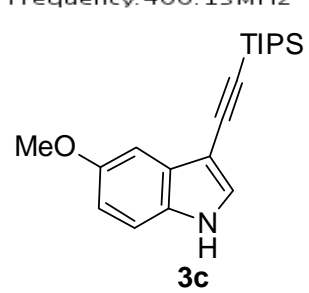

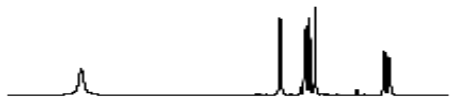

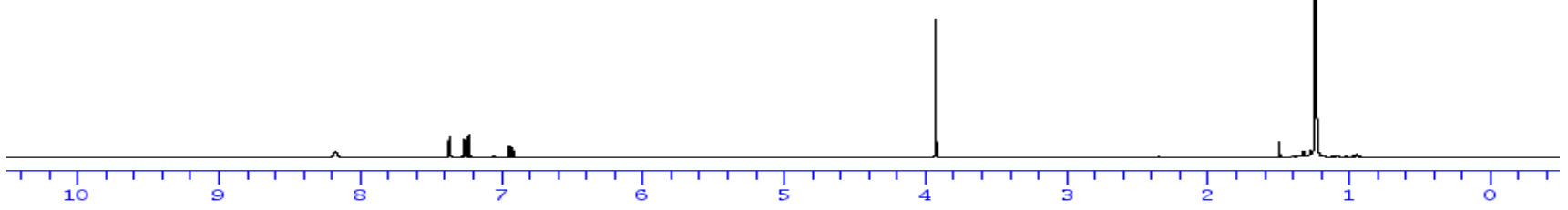

solvent: $<\mathrm{CDCI} 3>$
Frequency. $100.61 \mathrm{MHz}$
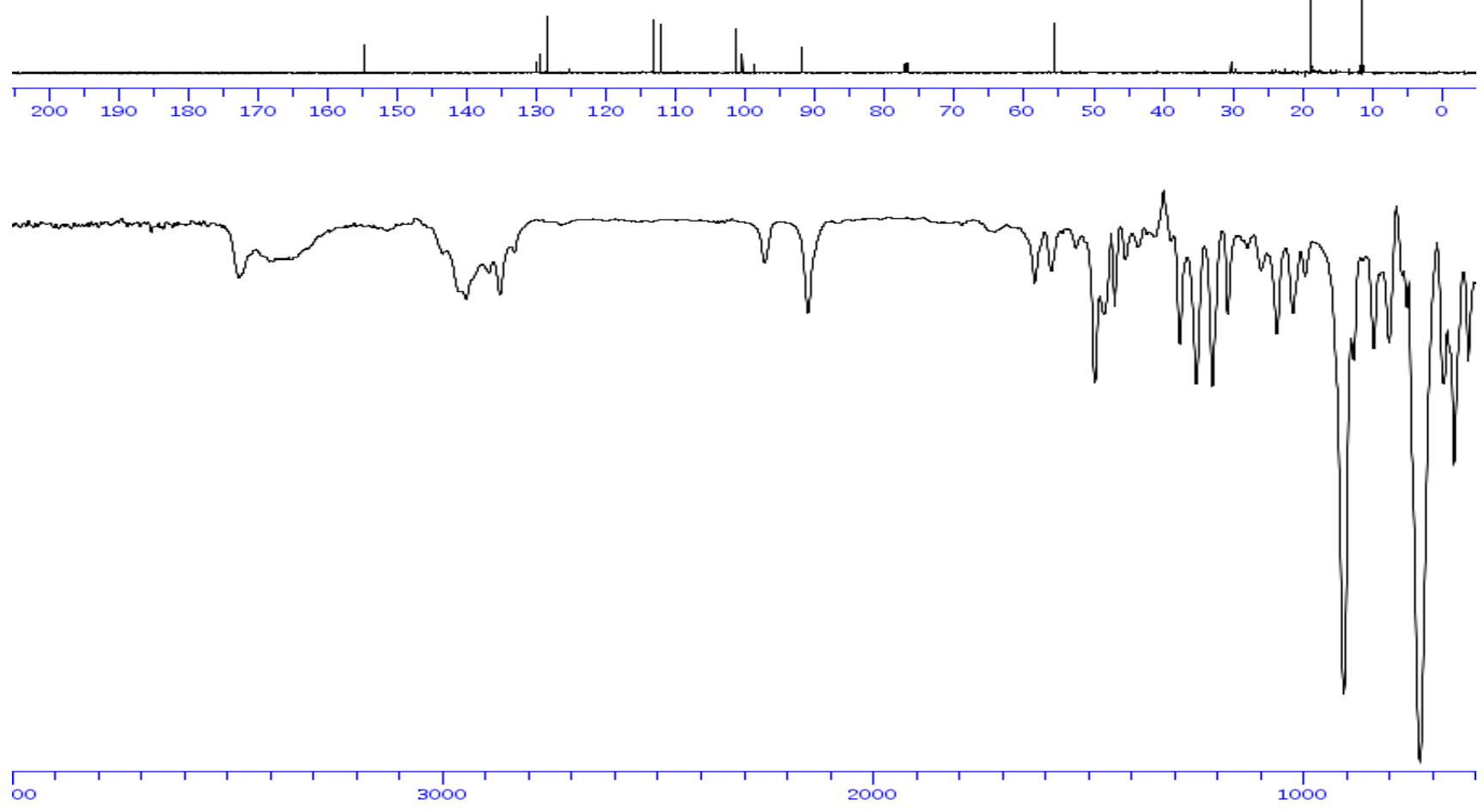

S30 


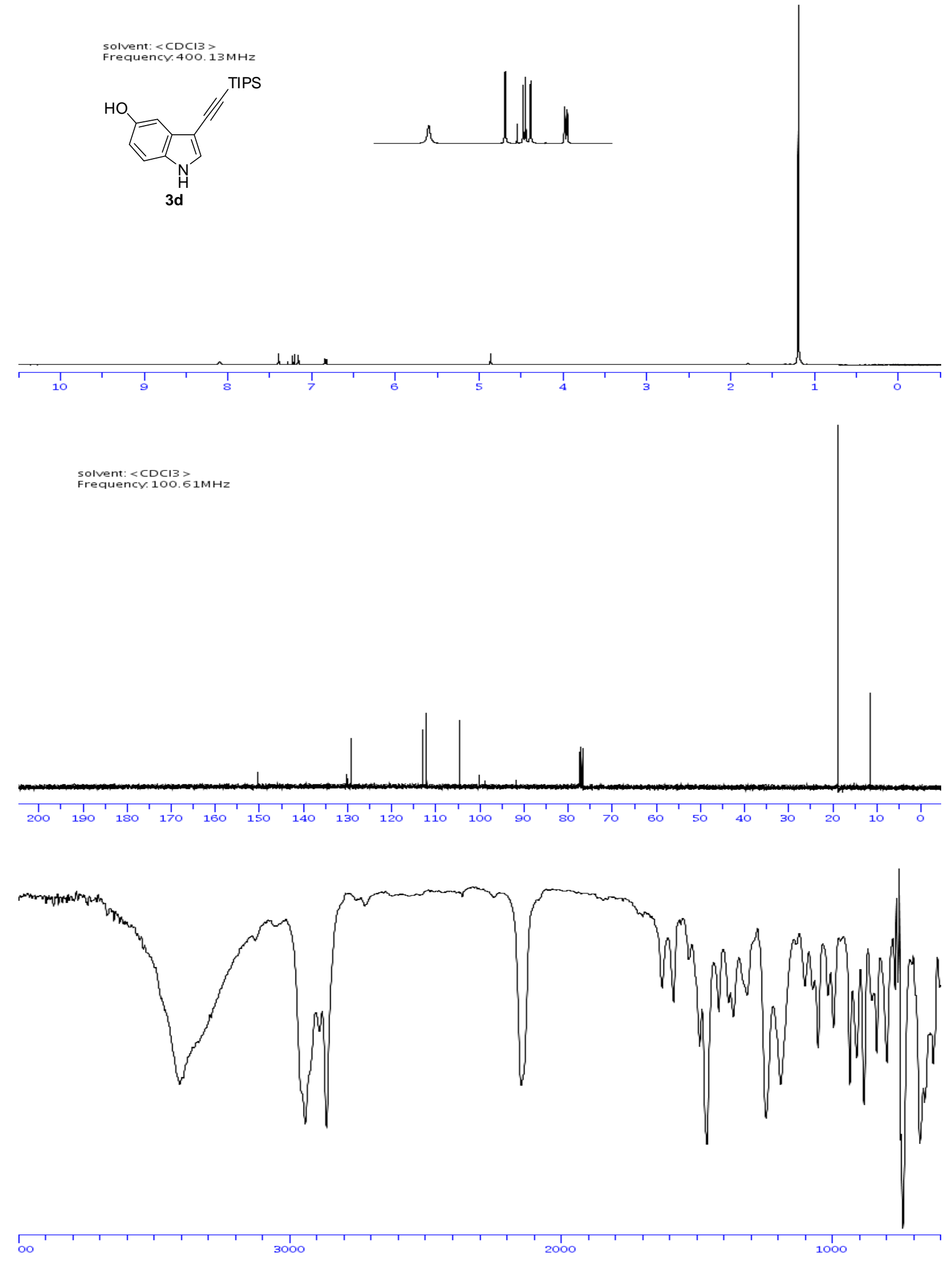




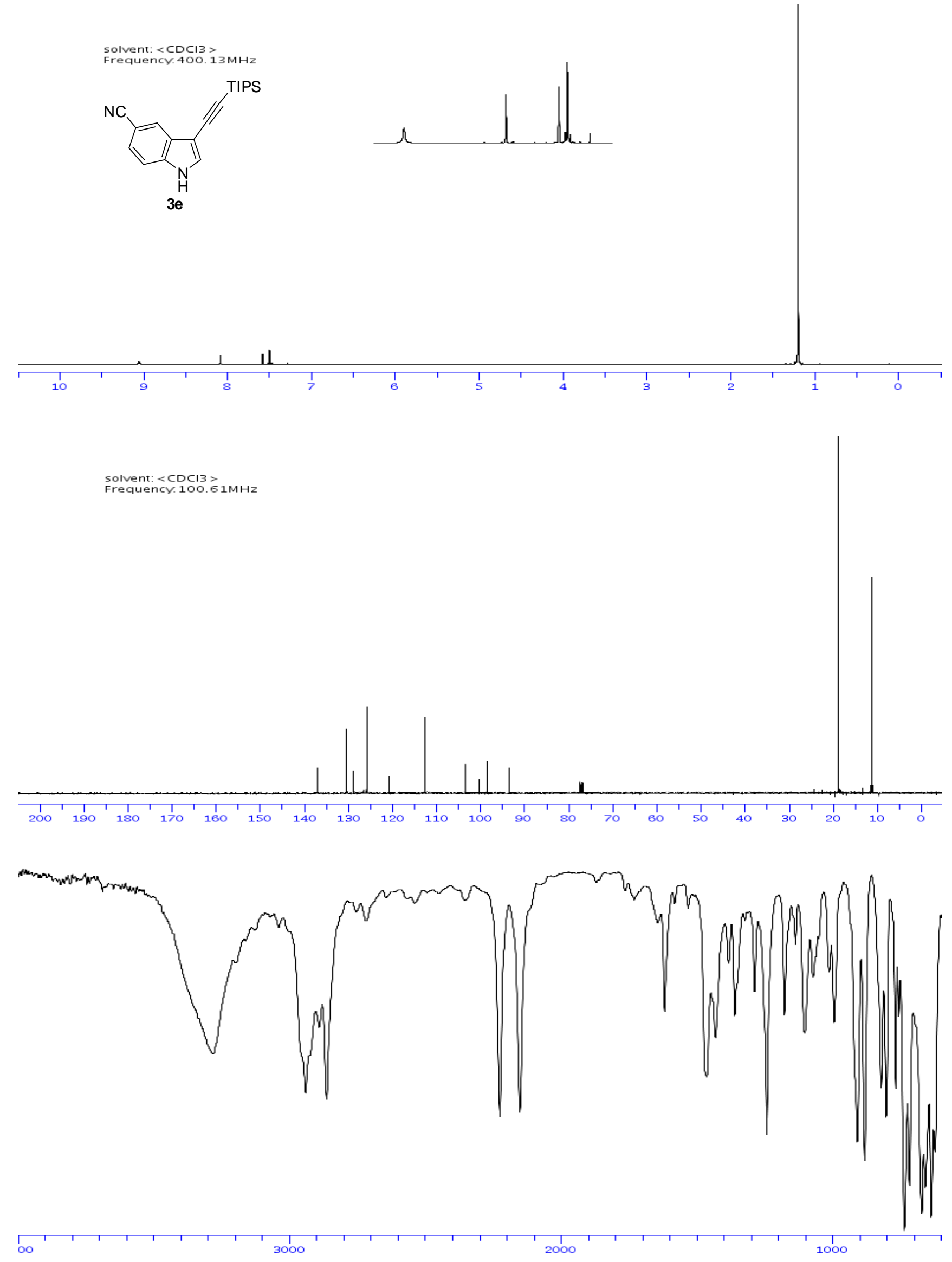


solvent: <MeOD

requency. 40O. $13 \mathrm{MHz}$

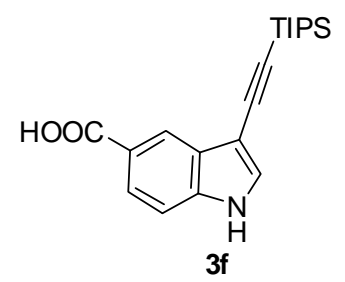
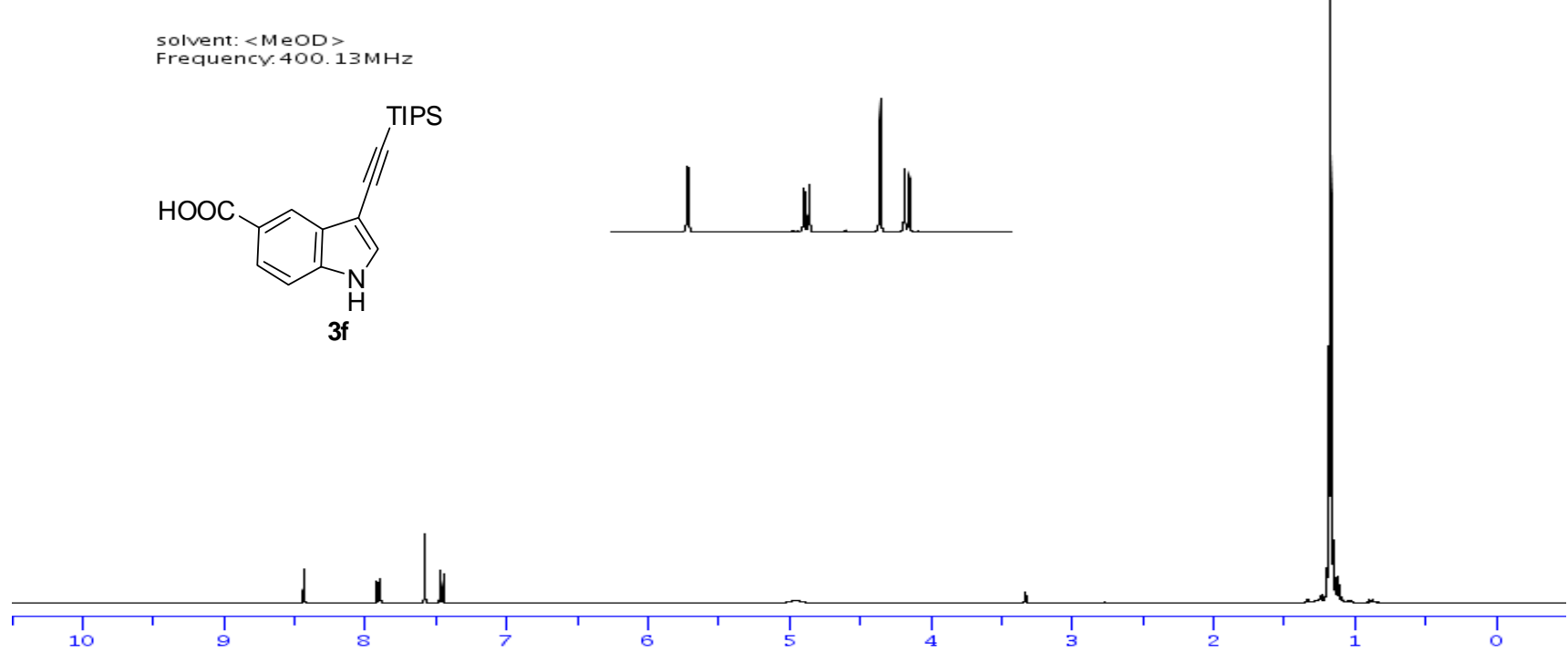

vent: $\langle$ MeOD $>$

Frequency. $100.61 \mathrm{MHz}$
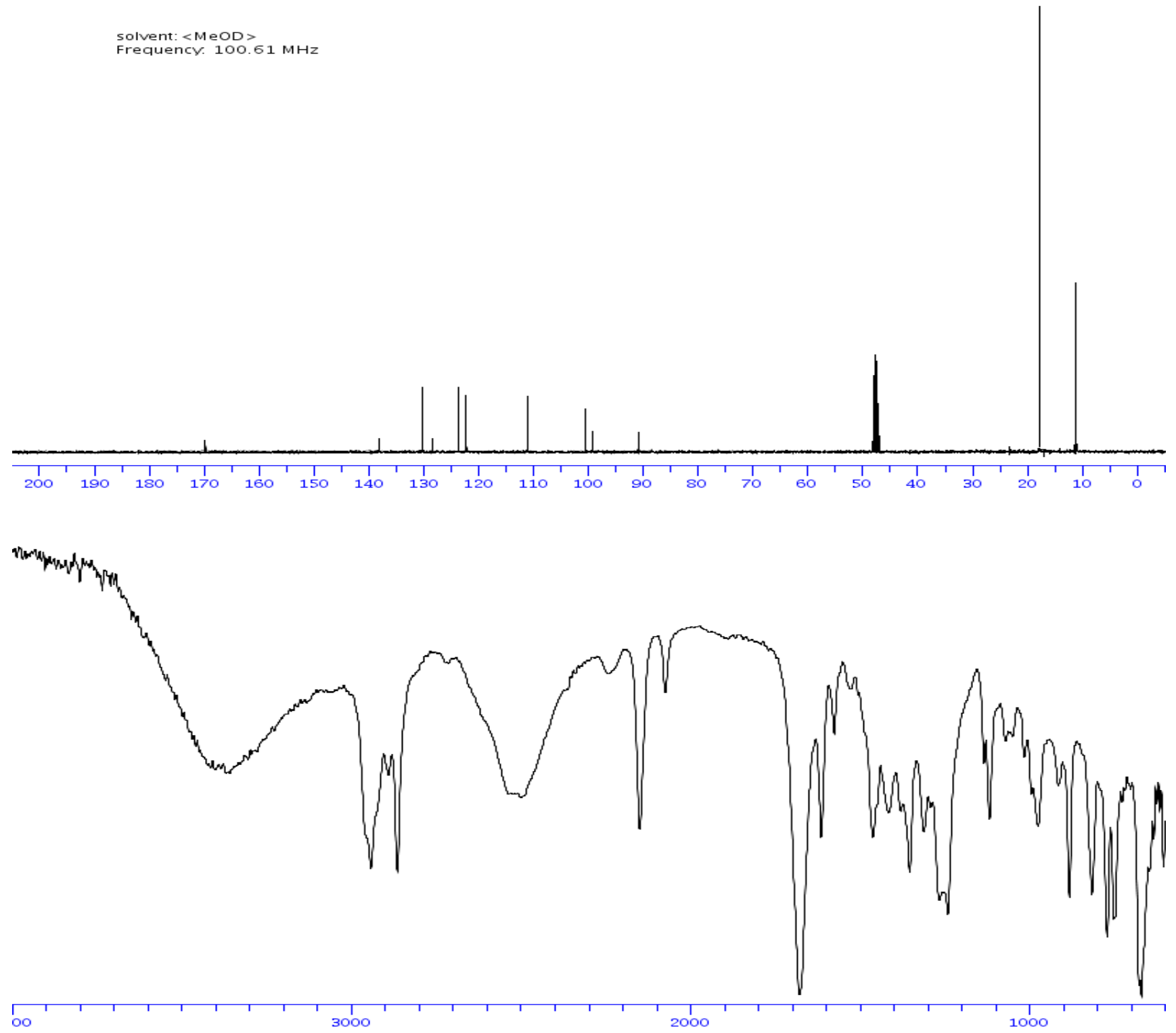
Frequency.400. $13 \mathrm{MHz}$

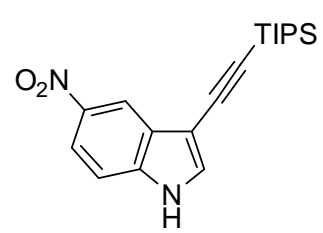

eld d.

3g

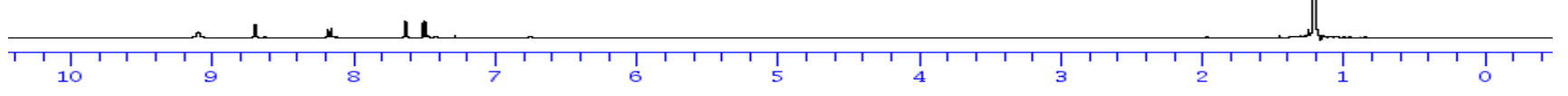

solvent: $<C D C 13>$
Frequency $100.61 \mathrm{MHz}$
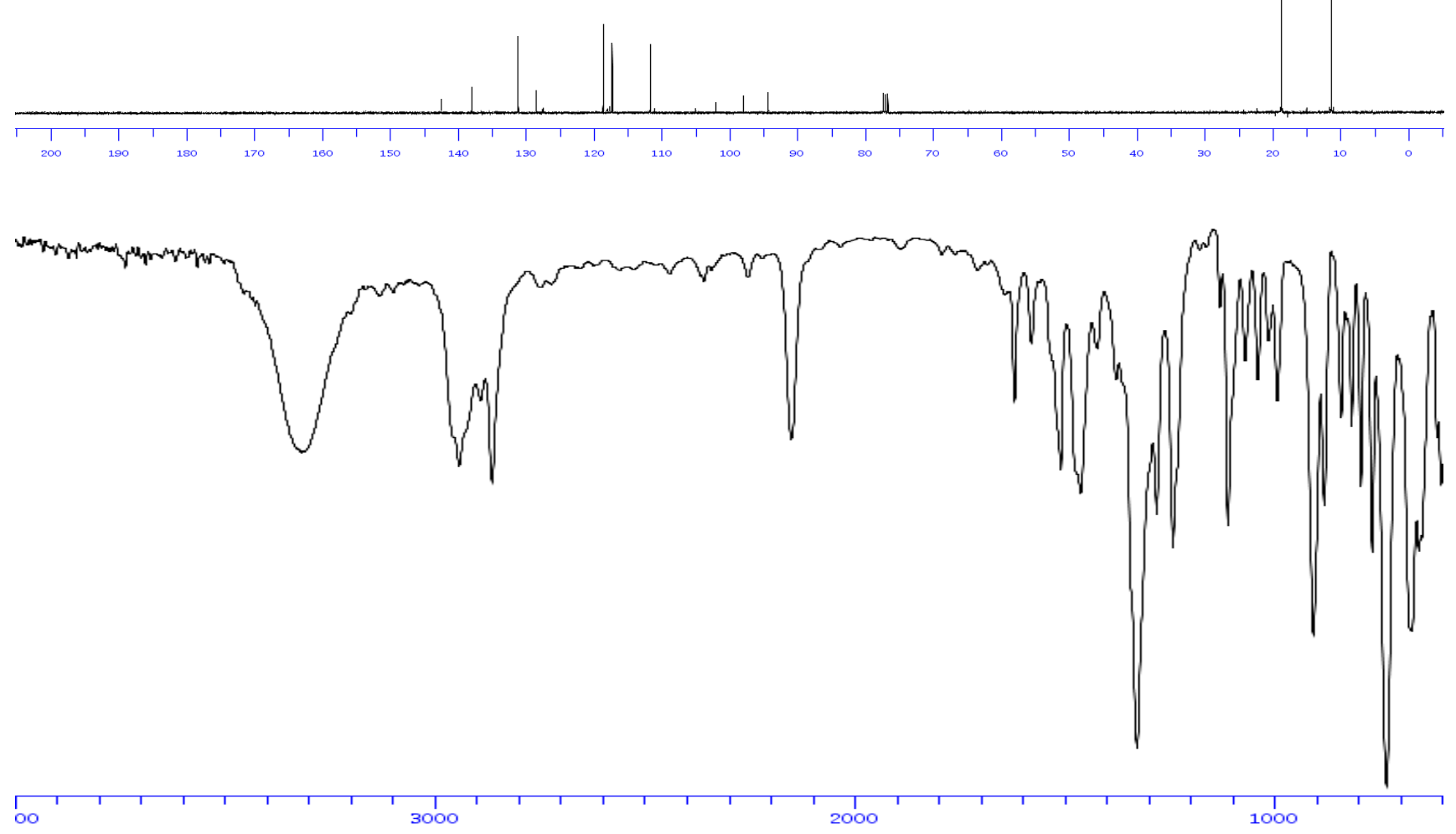

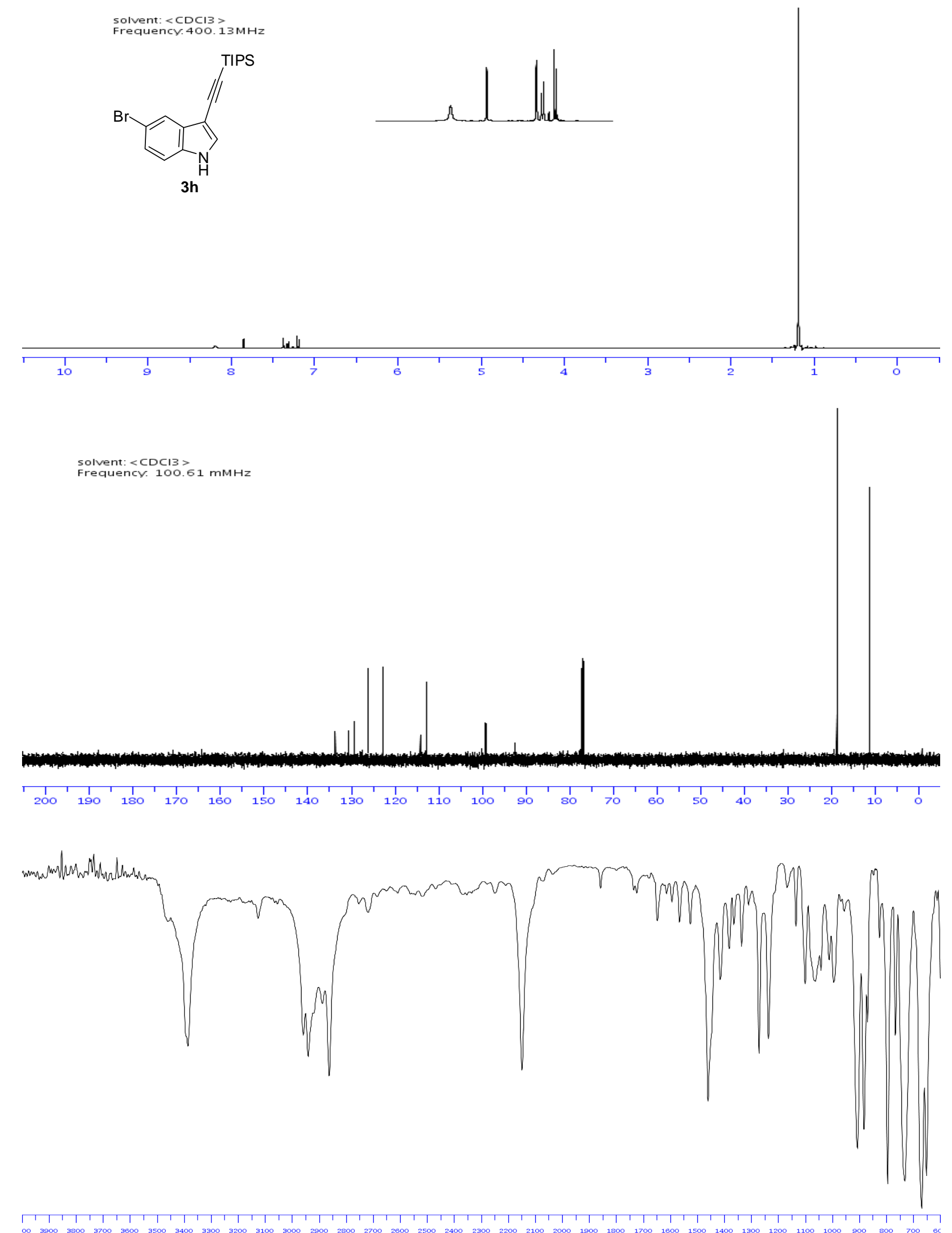

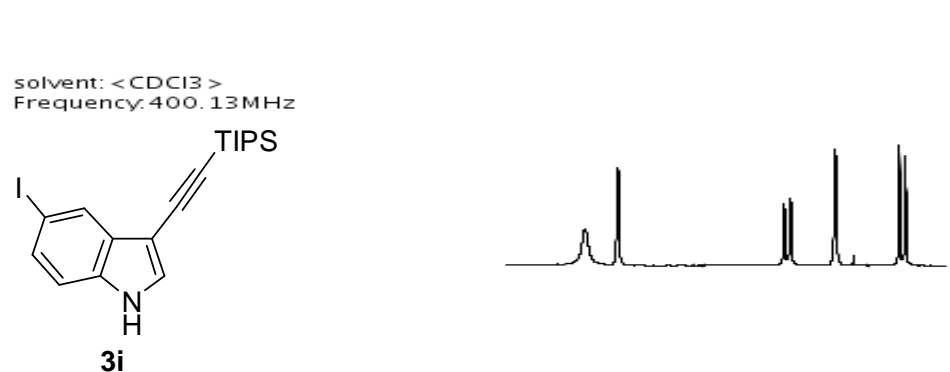

$3 \mathbf{i}$

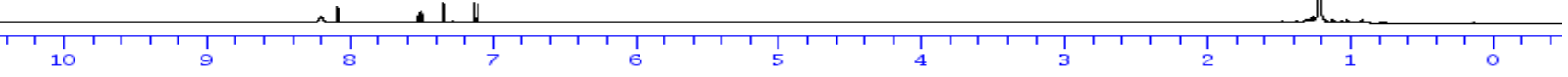

Solvent: $\angle C D C I 3>$
Frequency. $100.612769 \mathrm{MHz}$
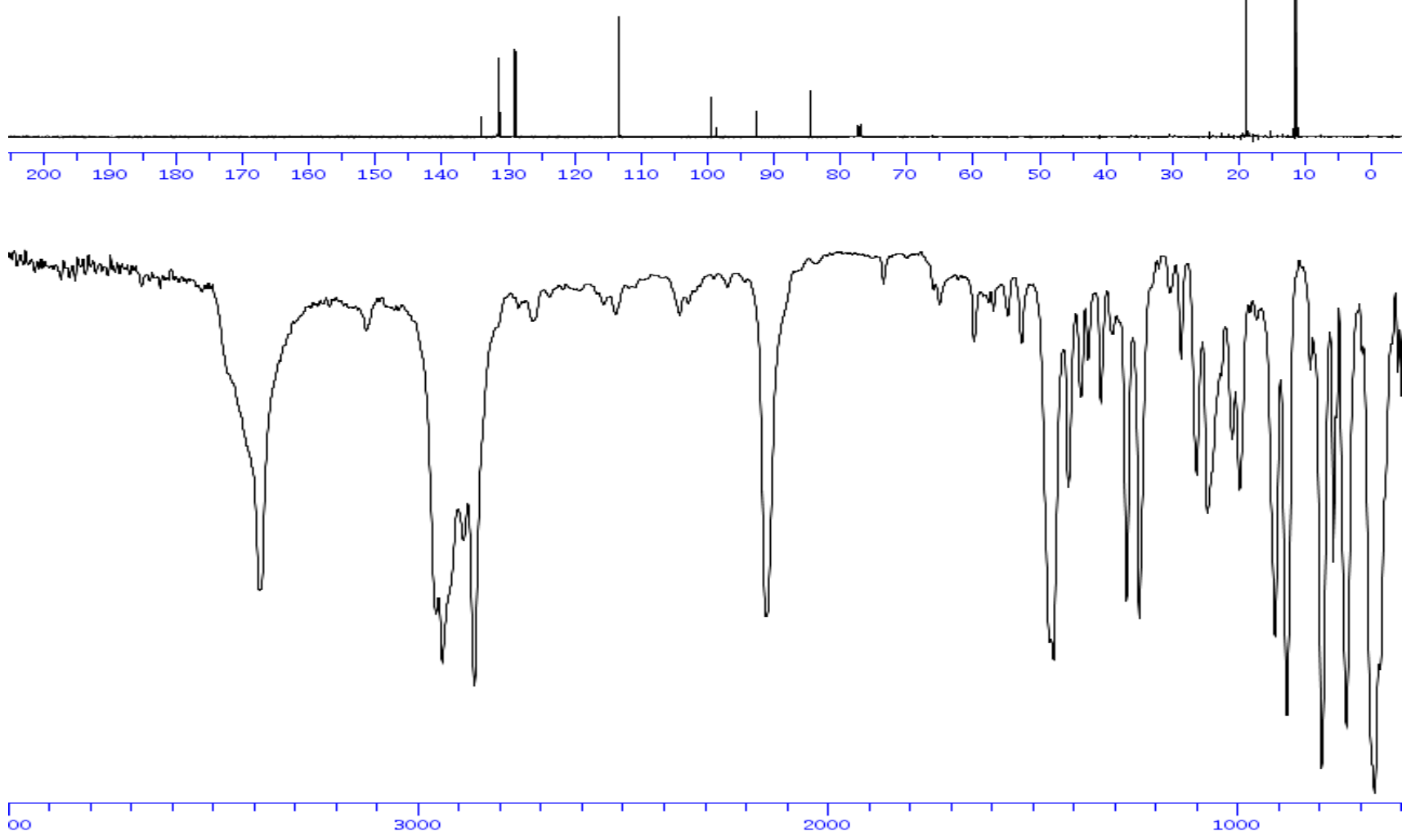

S36 


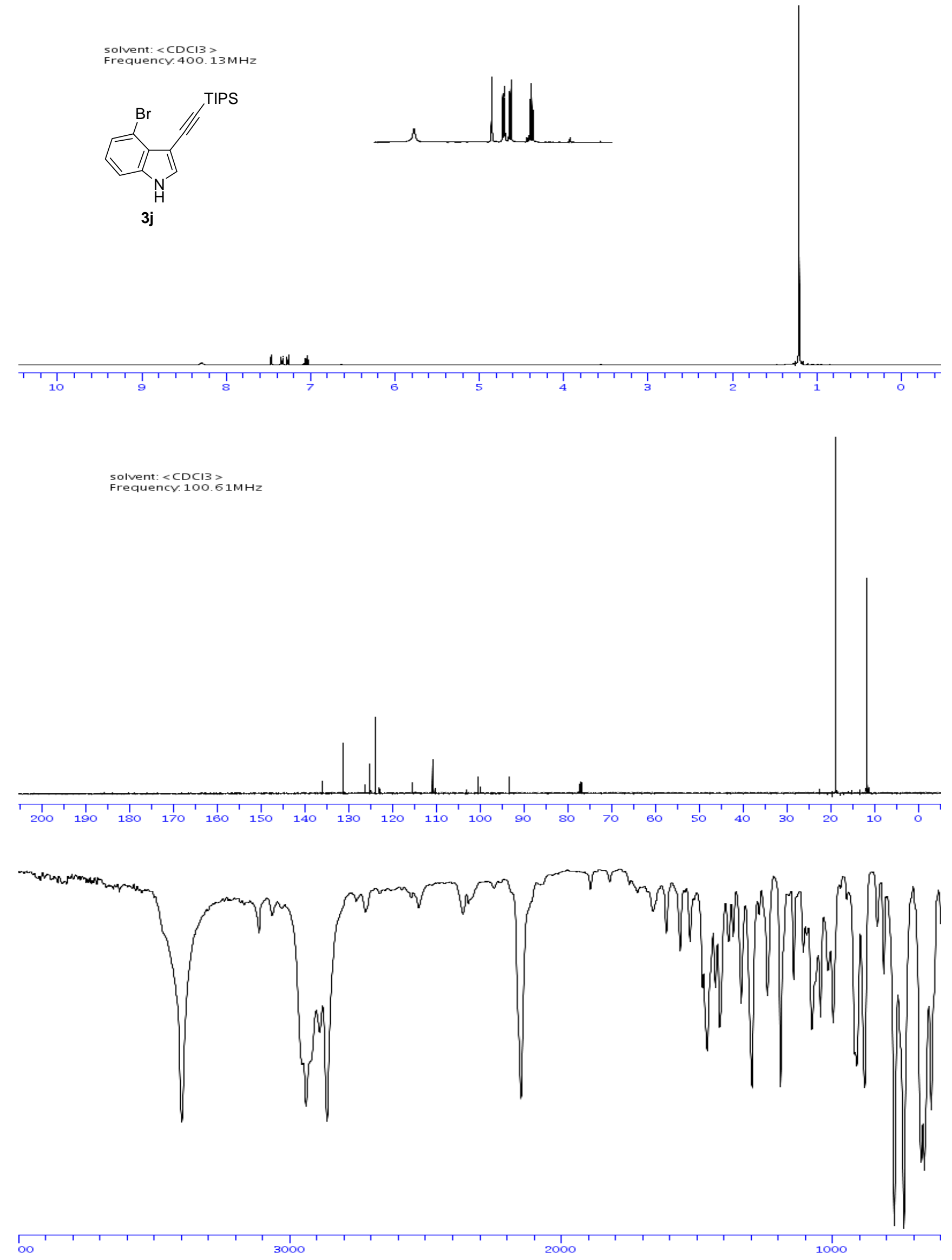



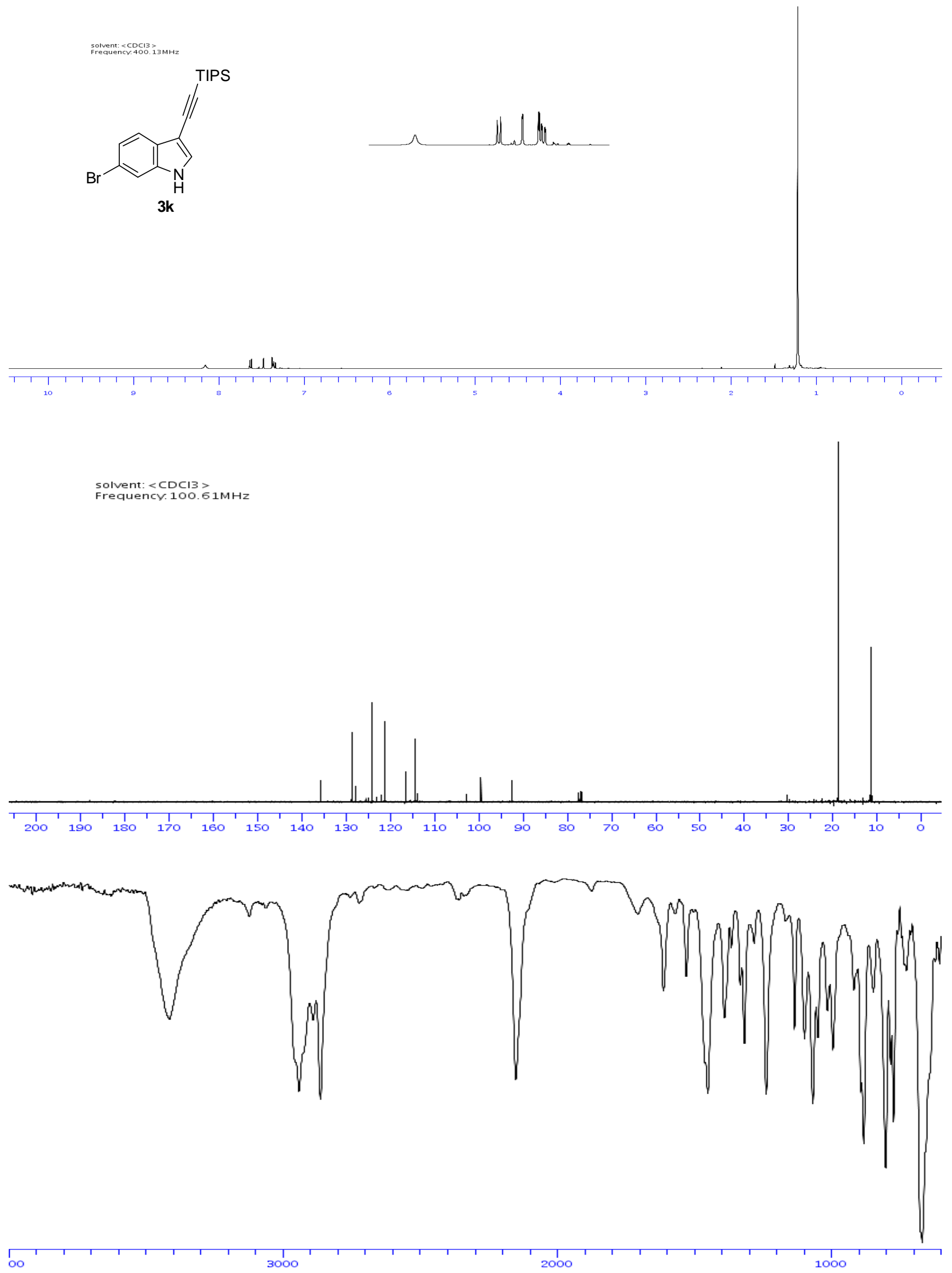
solvent: $\angle C D C I 3>$
Frequency.400. $13 \mathrm{MHz}$
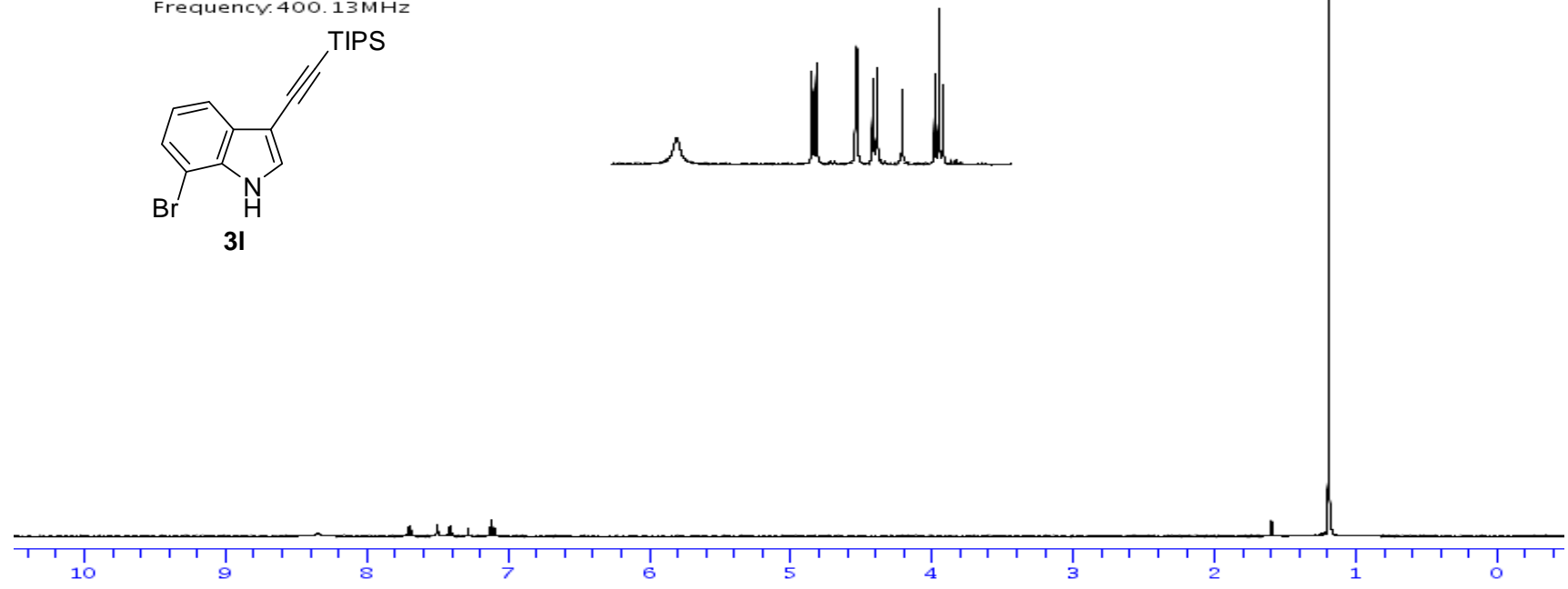

solvent: $\langle\mathrm{CDCI} 3\rangle$

Frequency. $100.612769 \mathrm{MHz}$
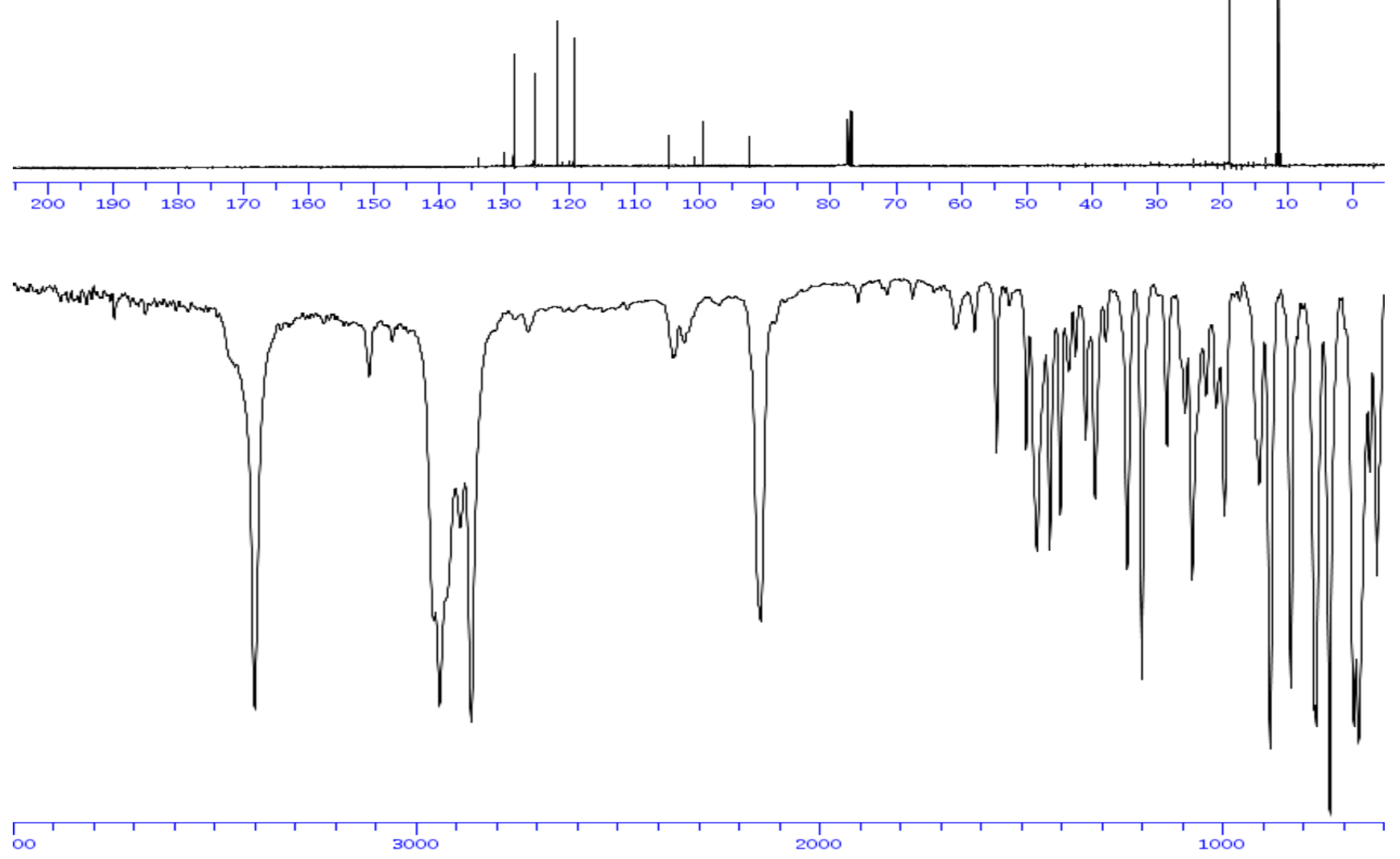

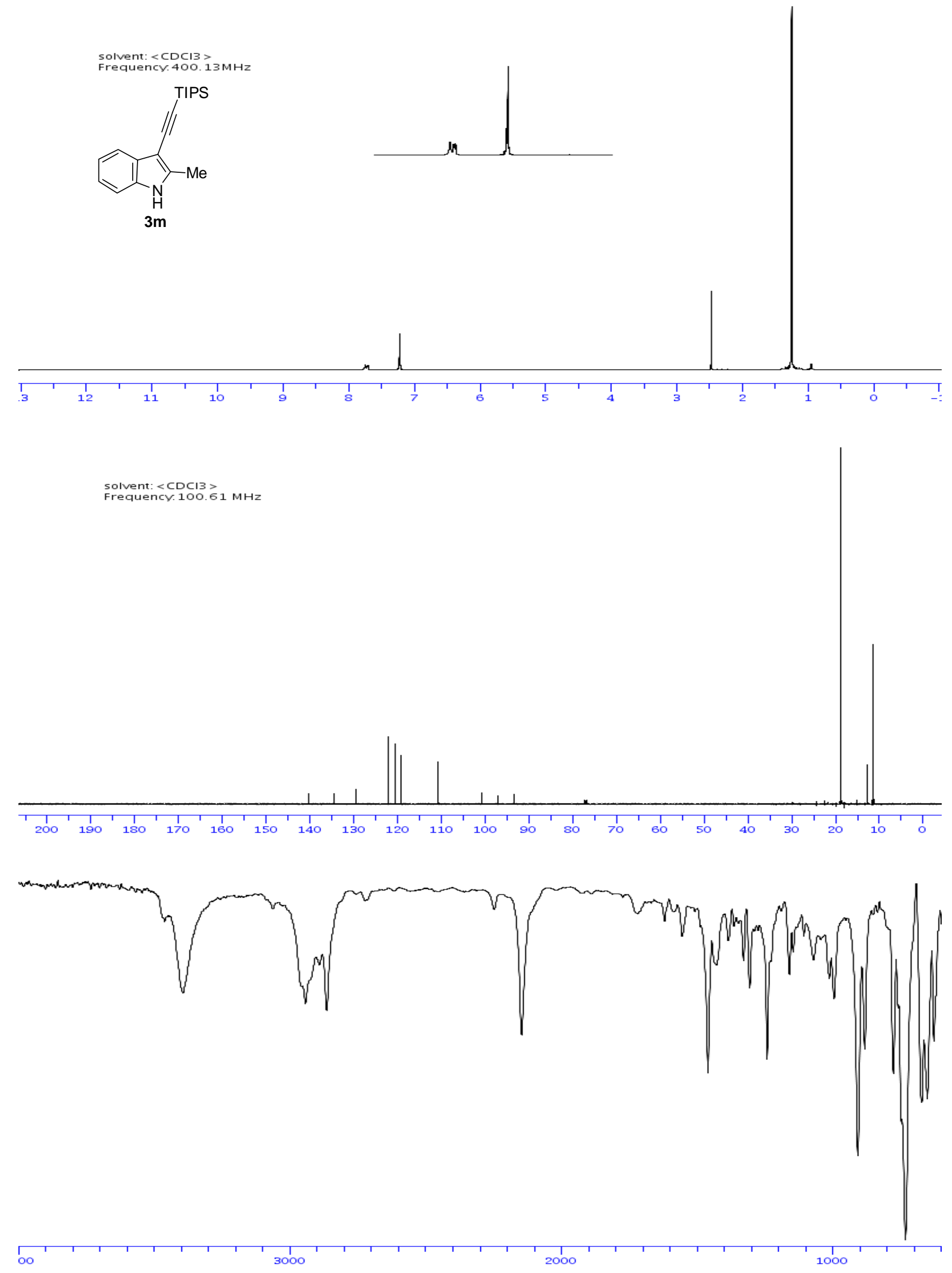


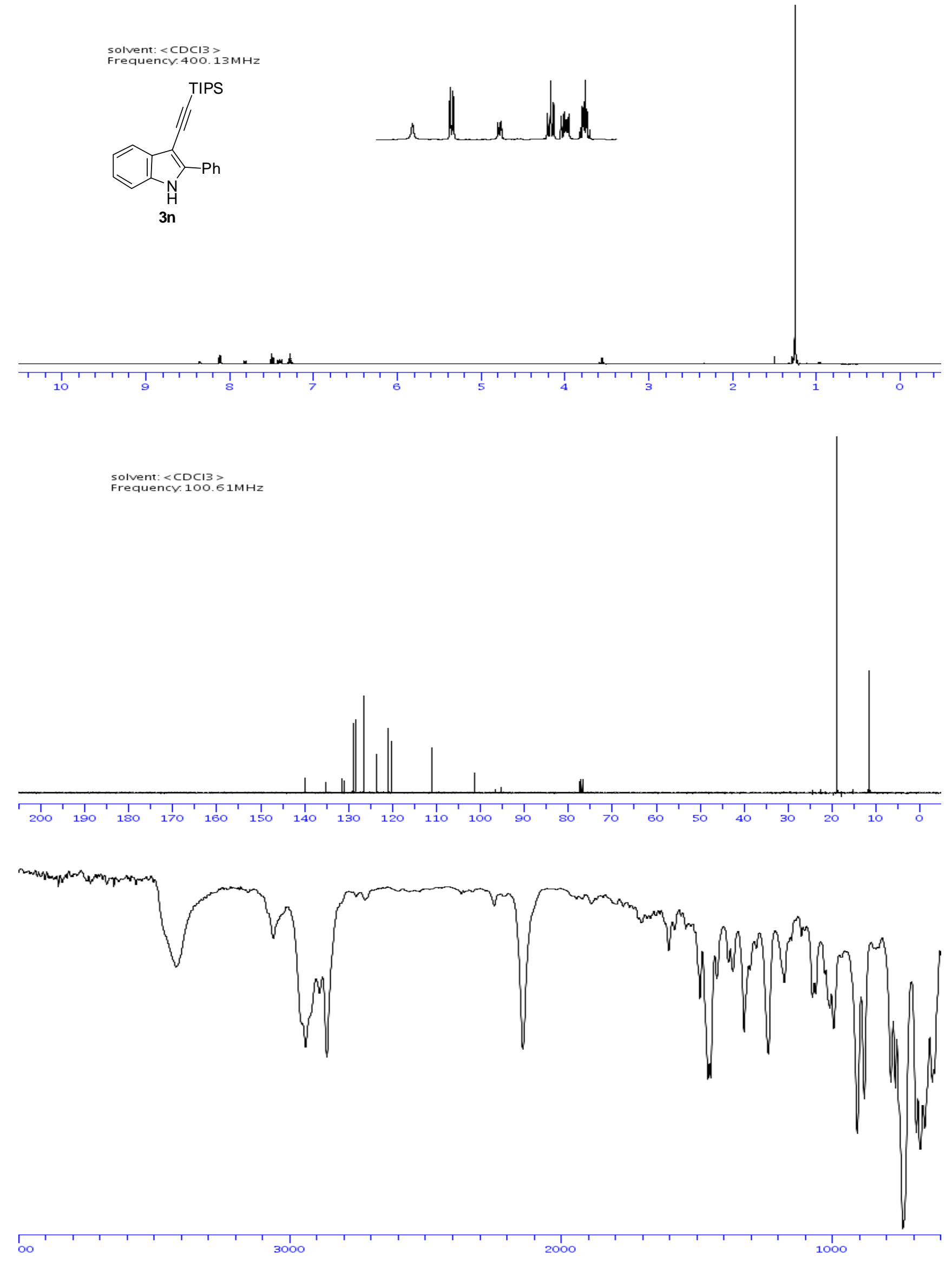




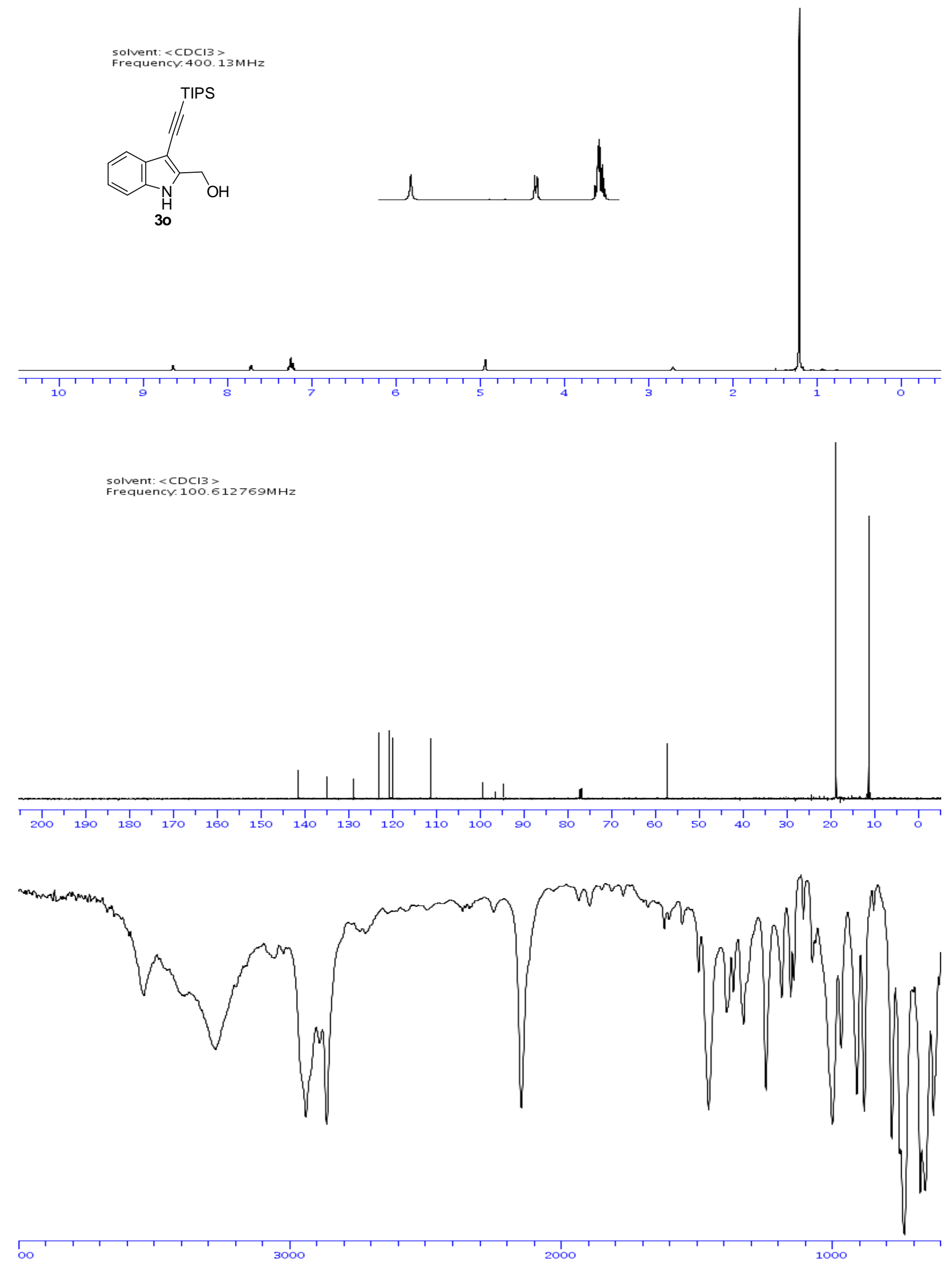



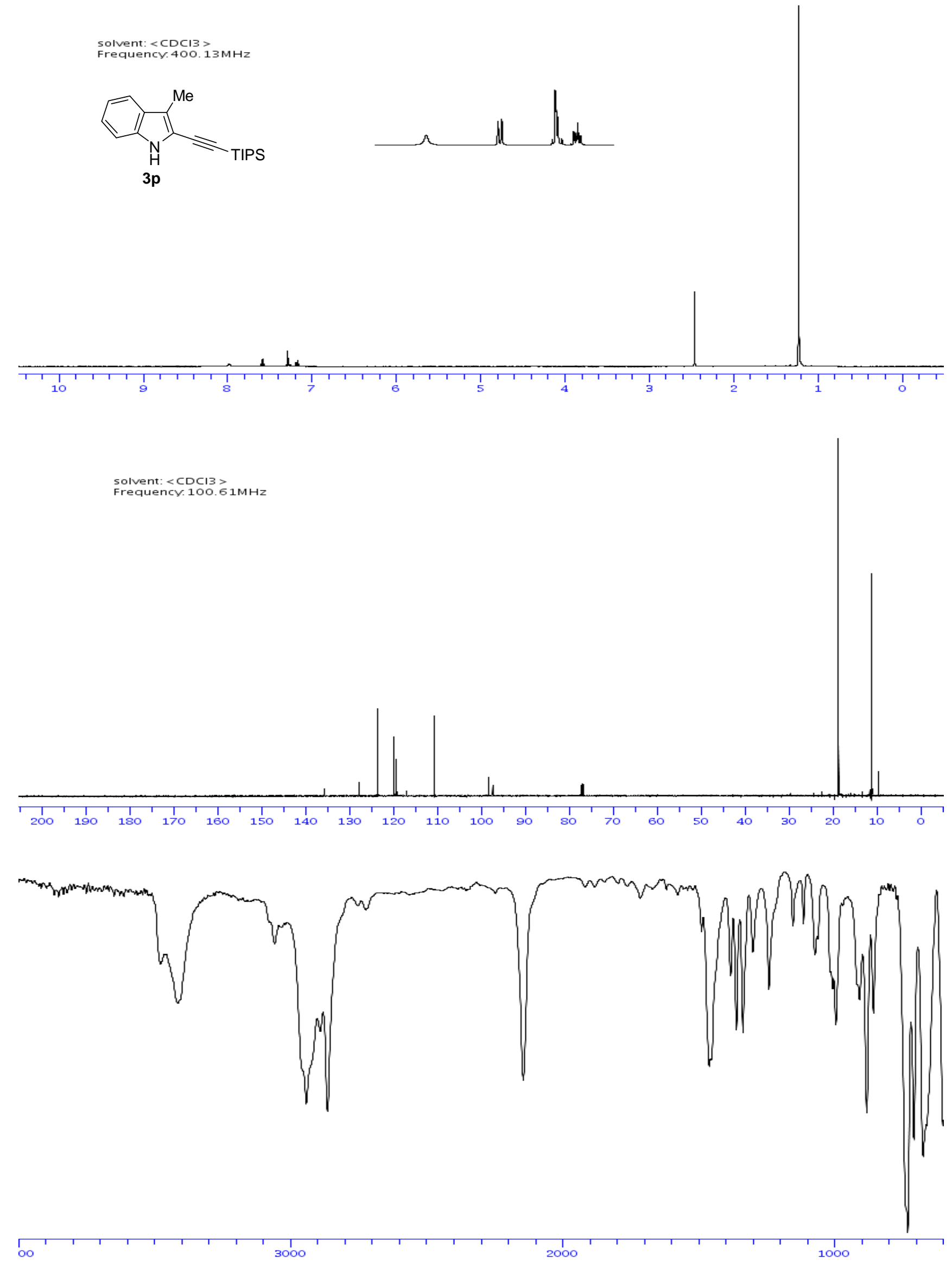
Frequency 400 . $3 \mathrm{MHz}$
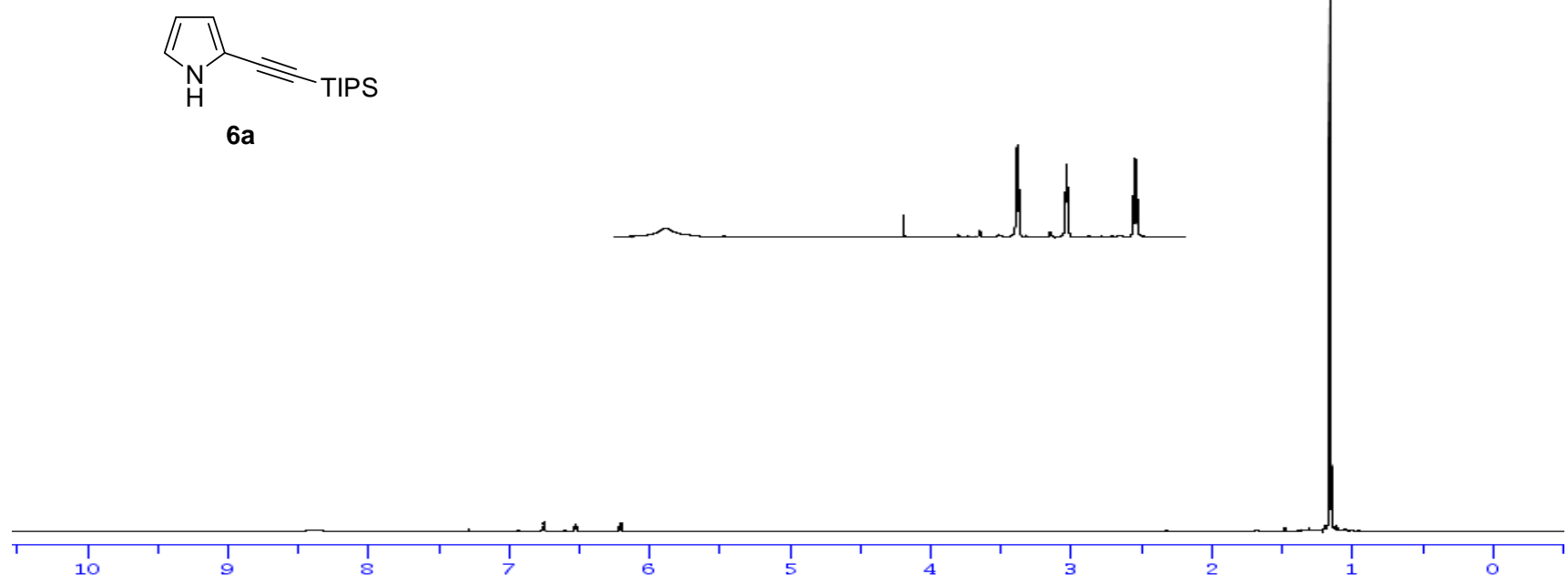

Solvent: $<C D C I 3>$
Frequency, $100.61 \mathrm{MHz}$
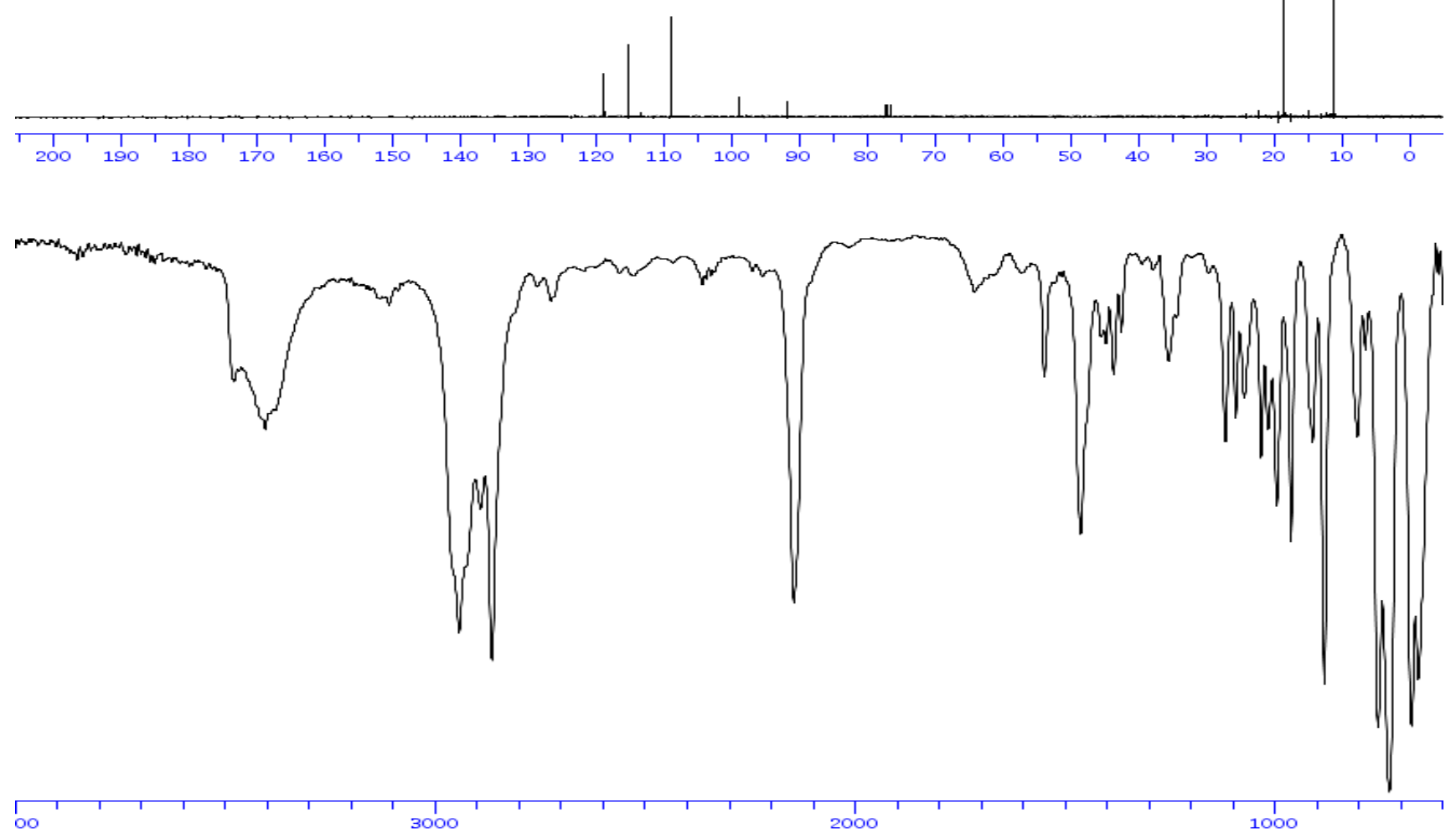
Frequency. 400.13MHz

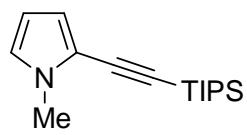

$6 b$
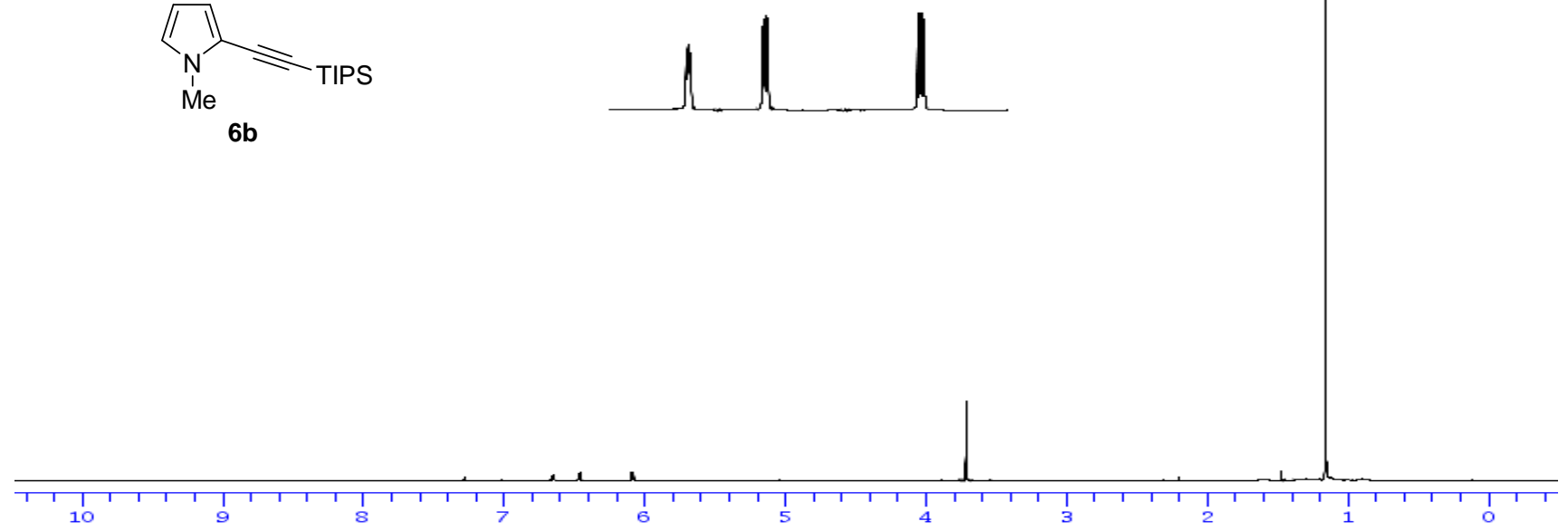

solvent: $\langle\mathrm{CDCI} 3\rangle$

Frequency. $100.61 \mathrm{MHz}$
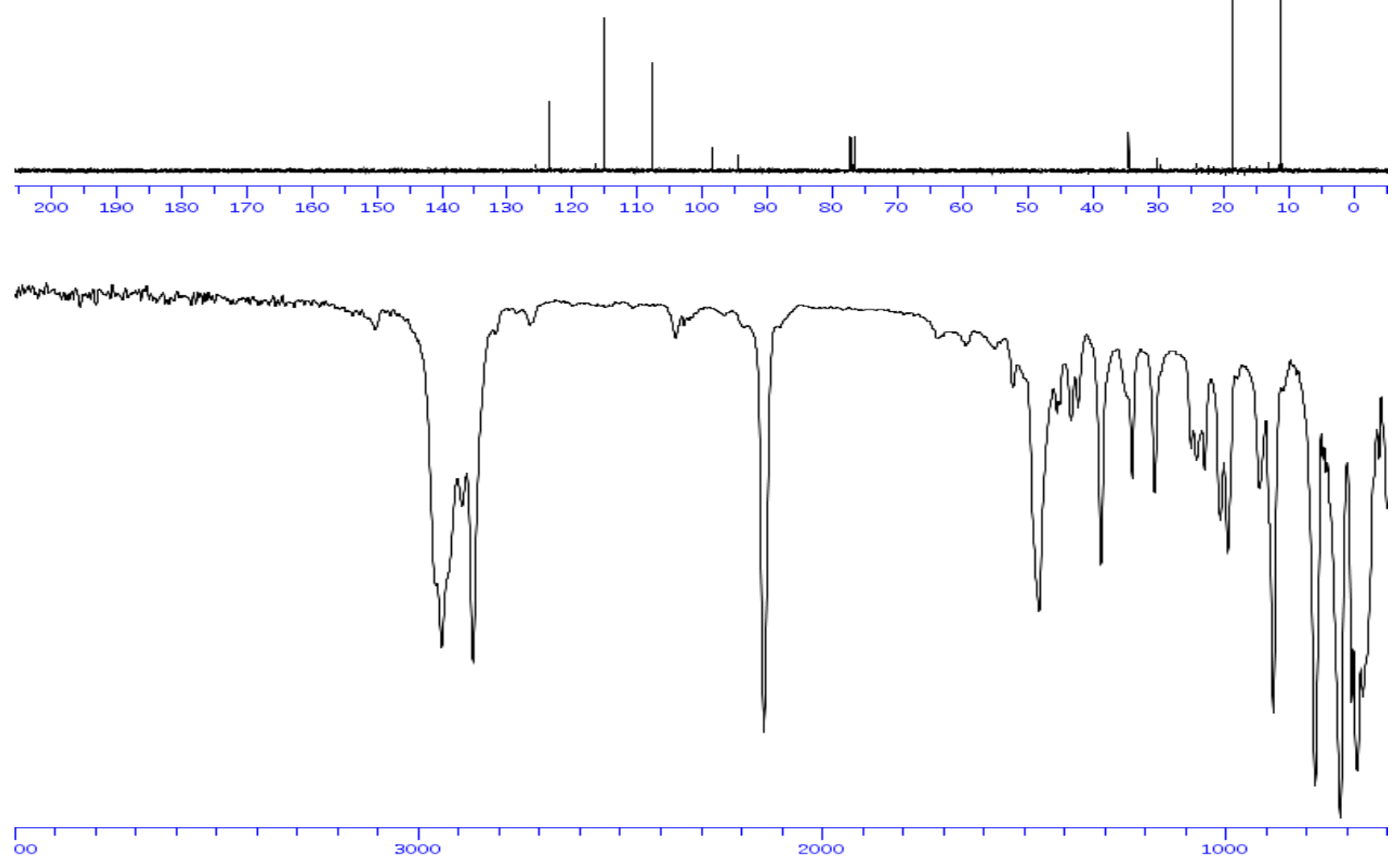

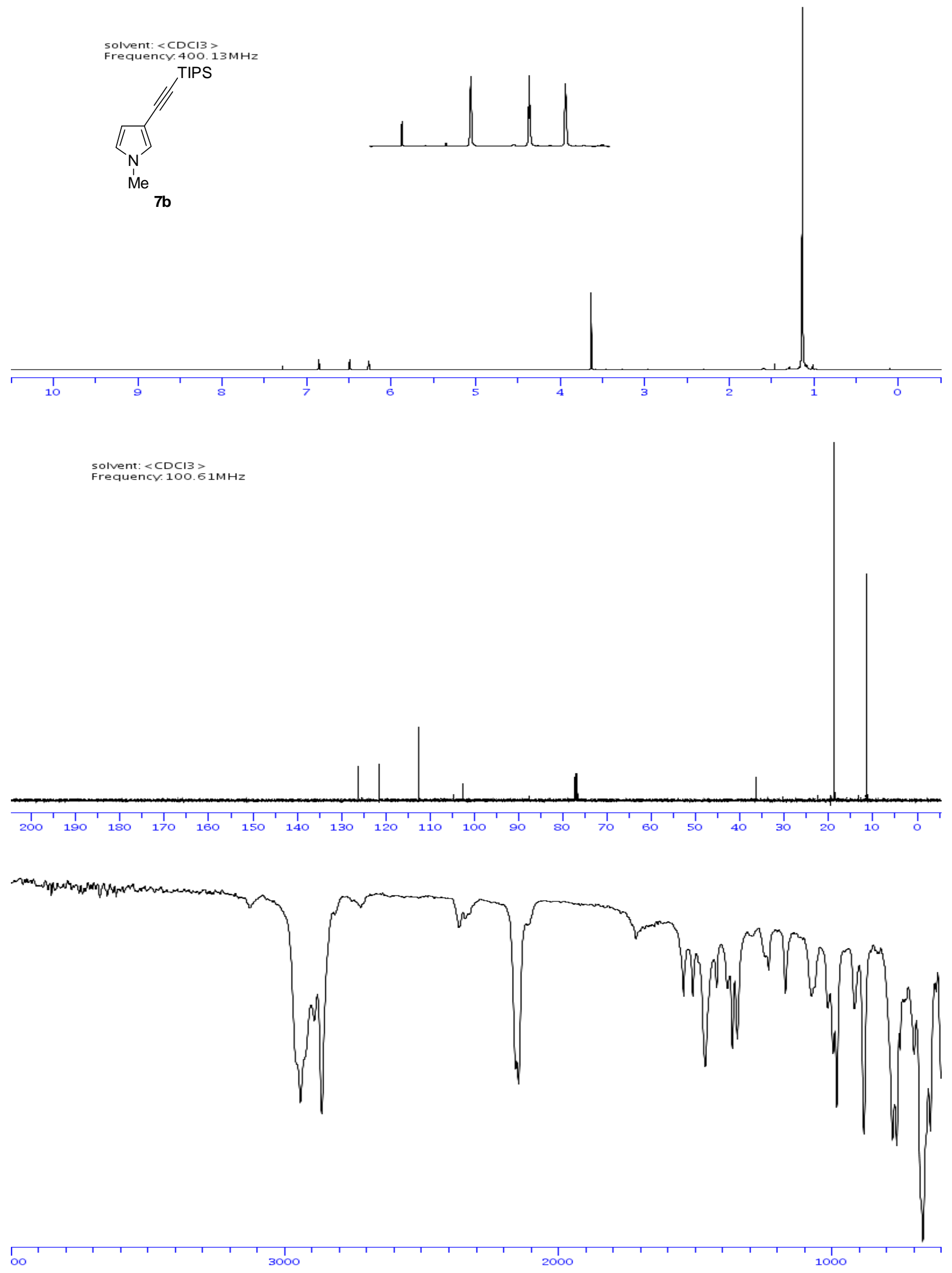


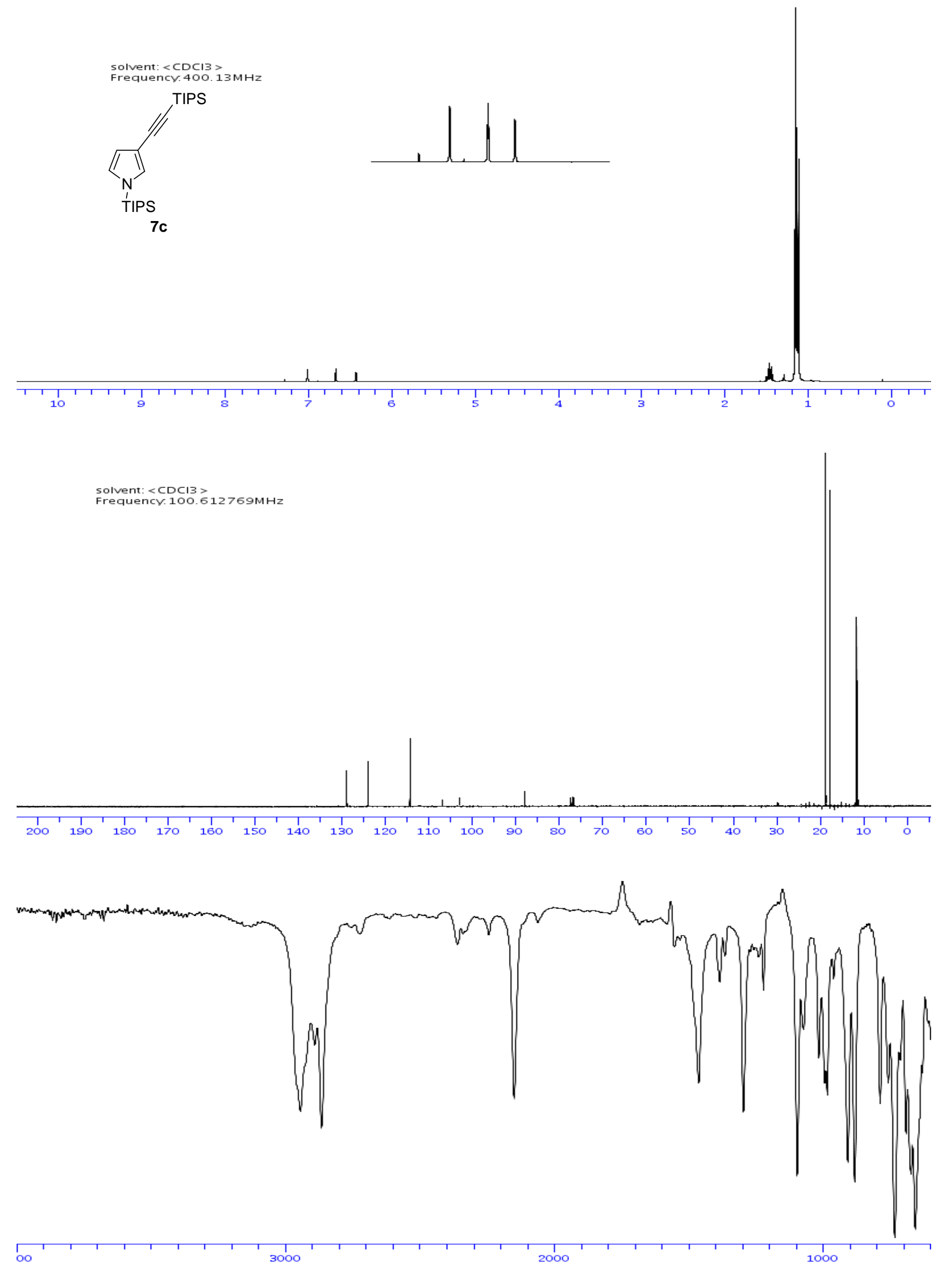


solvent: <CDCI3 >

Frequency.400.13MHz<smiles>CCc1ccc(C#CS[In])[nH]1</smiles>

$6 d$
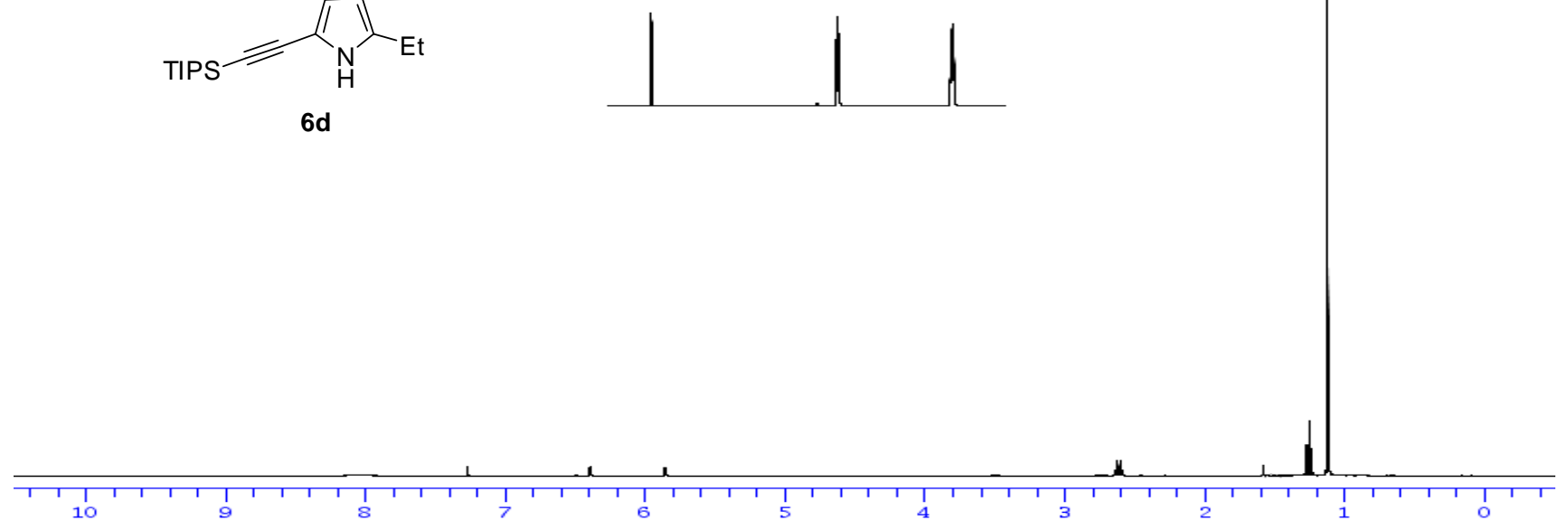

solvent: $<\mathrm{CDCI} 3$

Frequency. $100.61 \mathrm{MHz}$
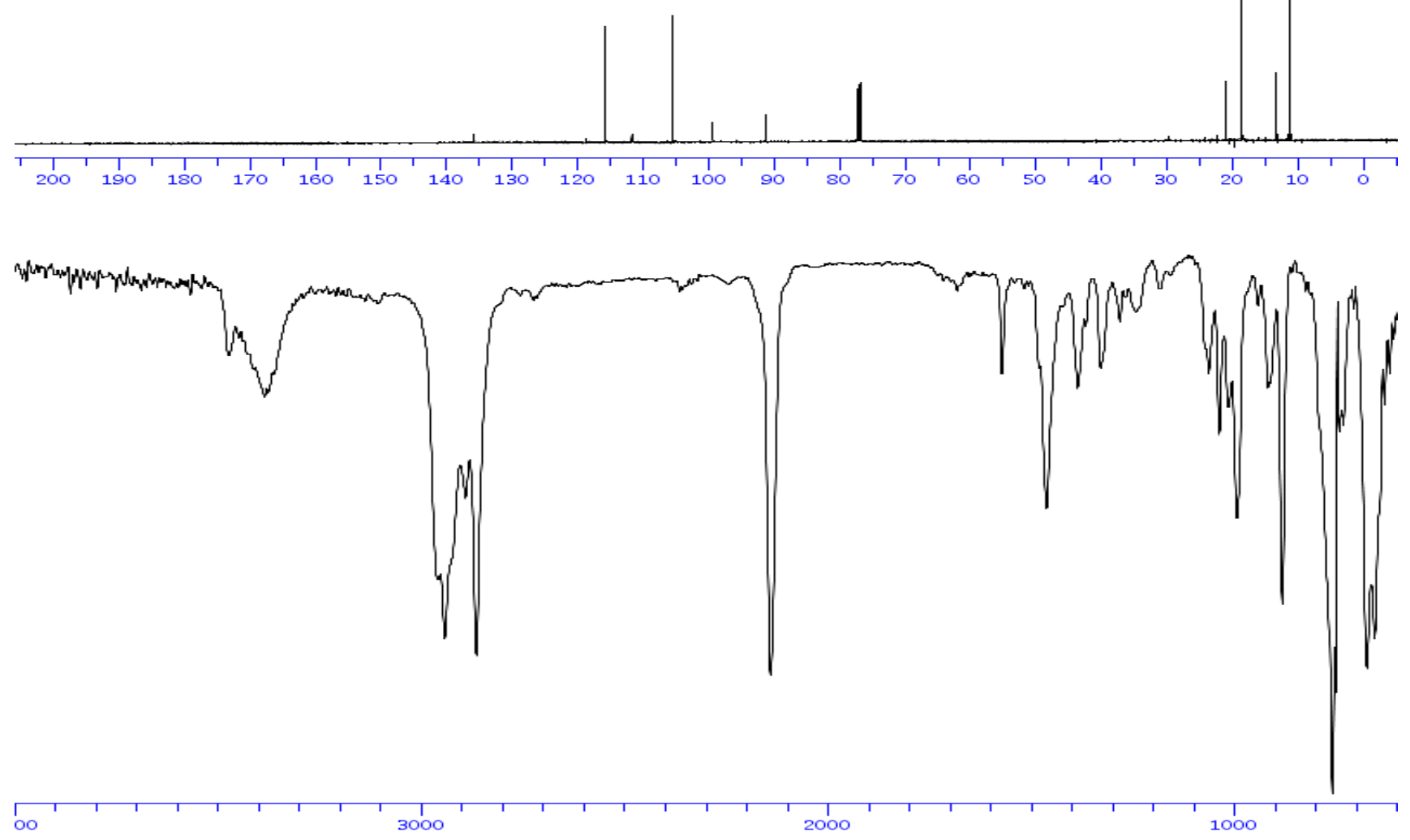


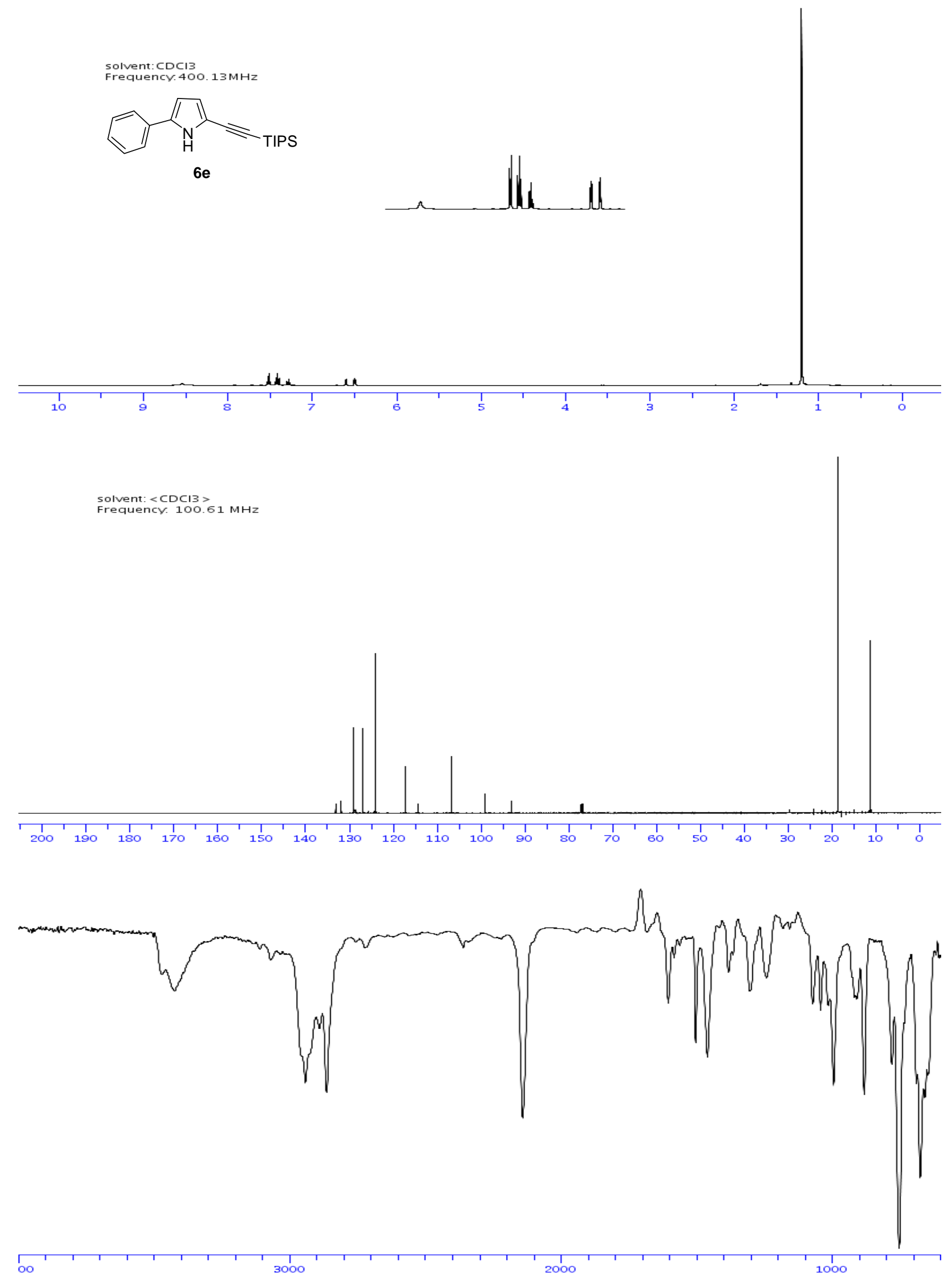


Solvent: $<\mathrm{CDCl} 3>$
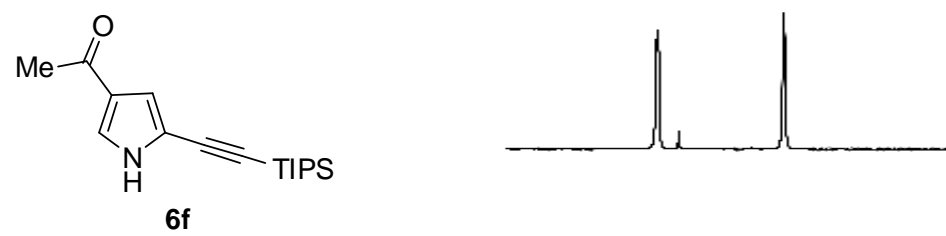

$6 f$

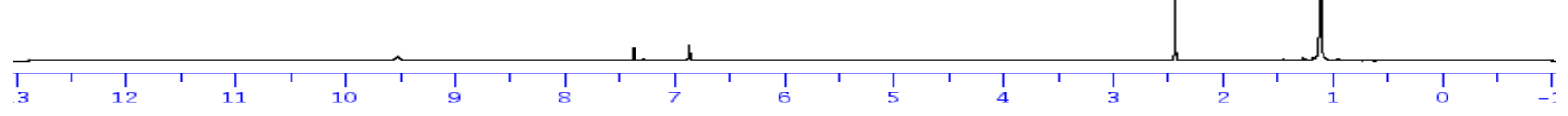

solvent: $<\mathrm{CDCl} 3\rangle$

Frequency. $100.61 \mathrm{MHz}$
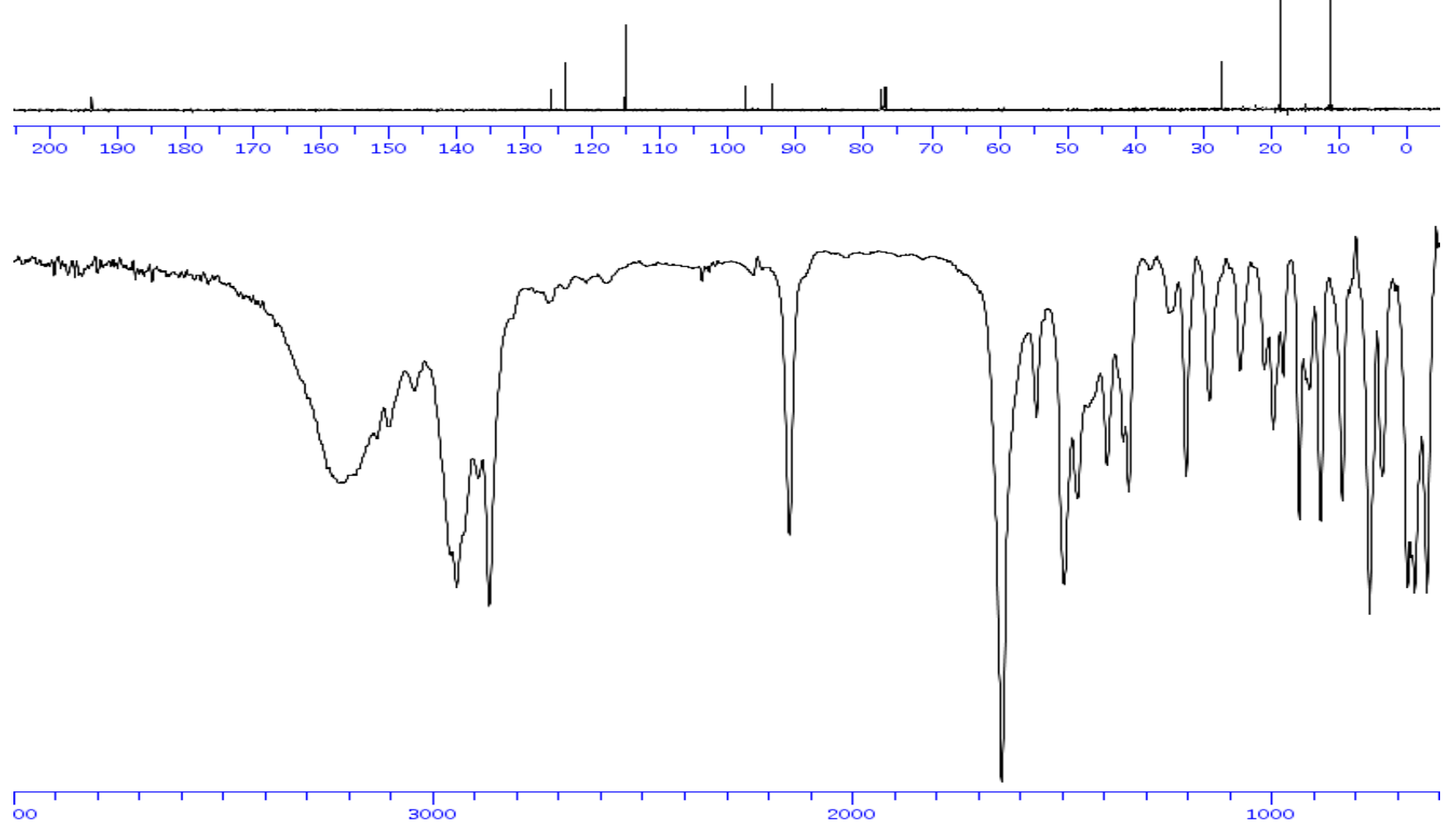
solvent: <CDCI3 >

Frequency. $400.13 \mathrm{MHz}$
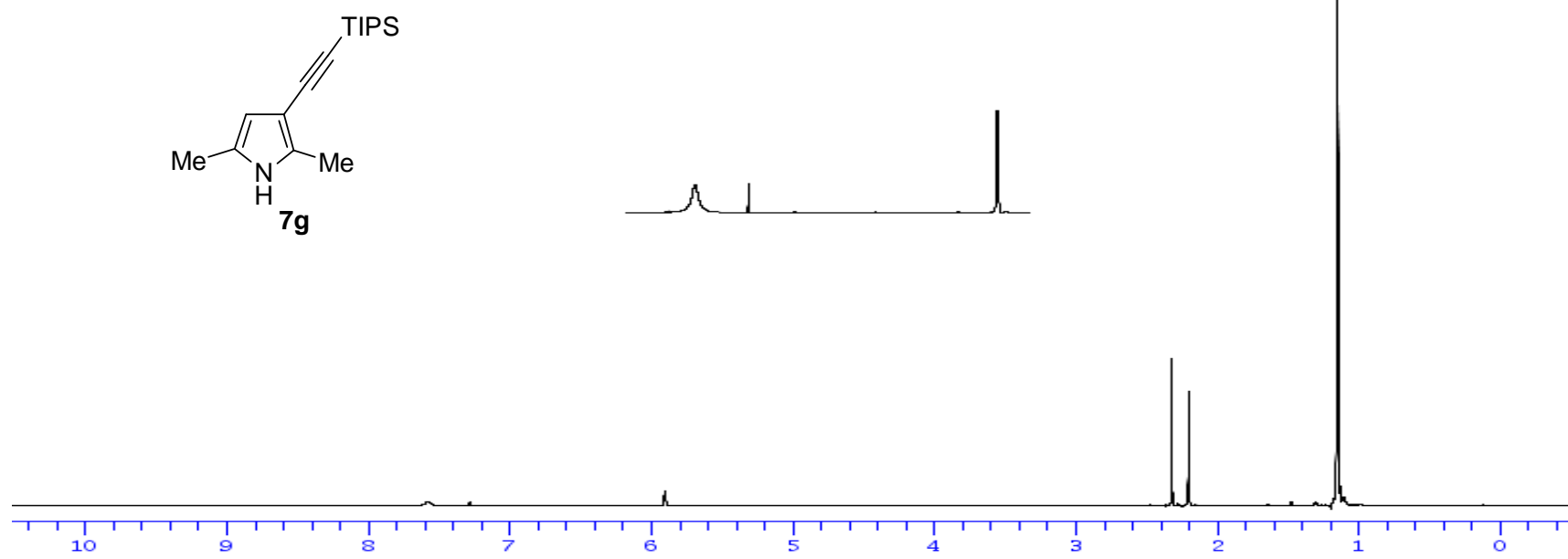

solvent: $<C D C I 3>$
Frequency. $100.61 \mathrm{MHz}$
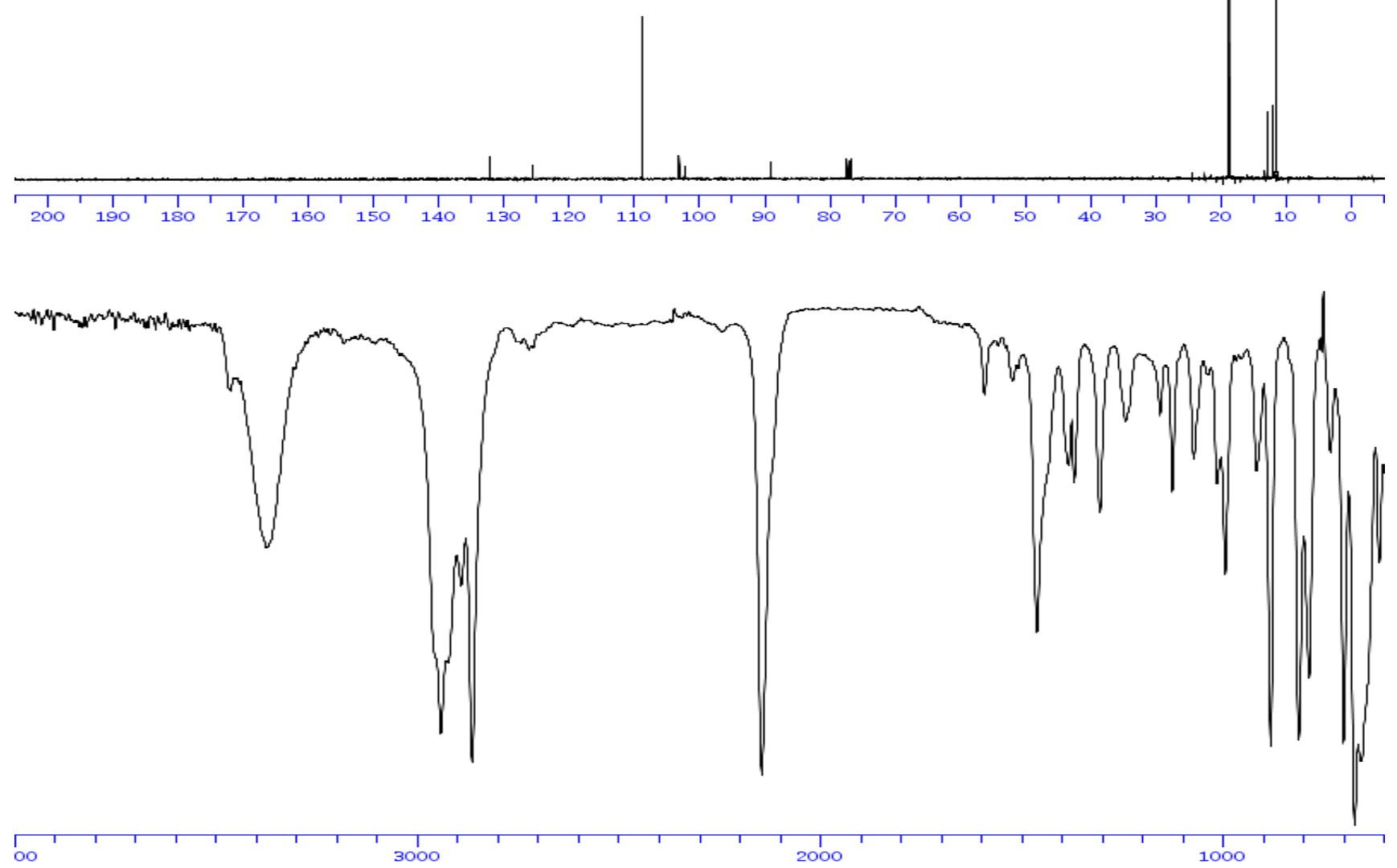
solvent: $<\mathrm{CDCI} 3$

Frequency. $400.13 \mathrm{MHz}$

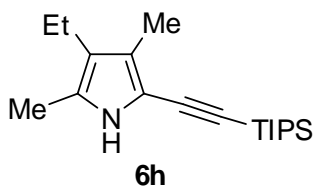

$6 h$

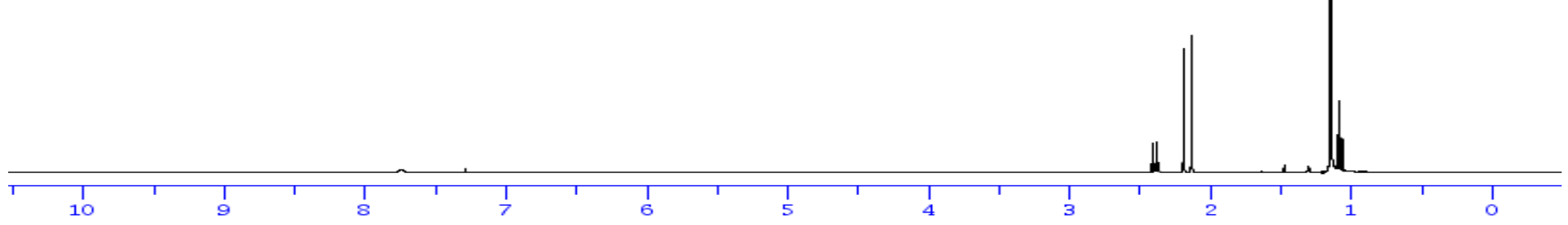

solvent: $<C D C I 3>$

Frequency. $100.61 \mathrm{MHz}$
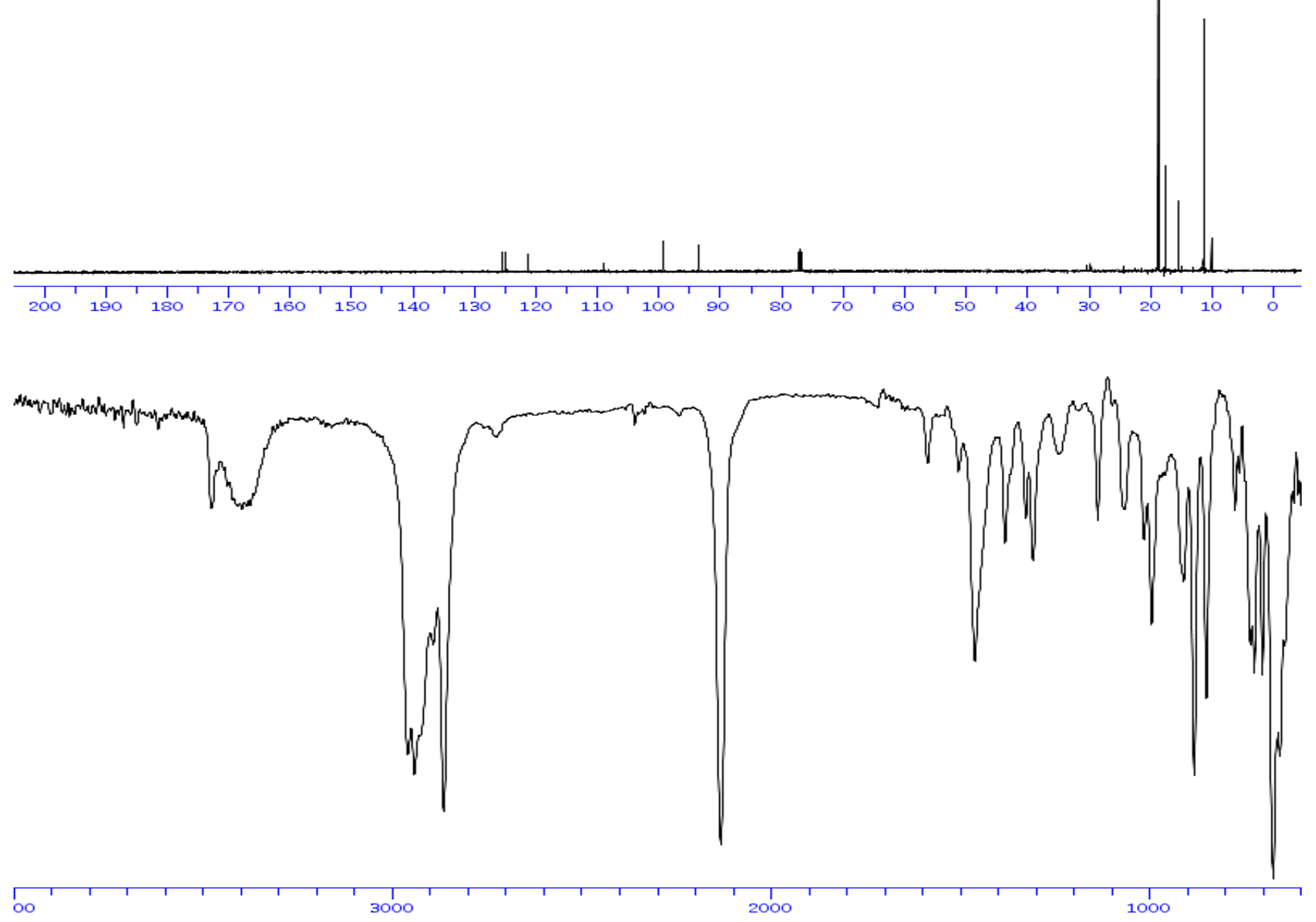
solvent: $\langle\mathrm{CDCI} 3>$

Frequencr $400.13 \mathrm{MHz}$

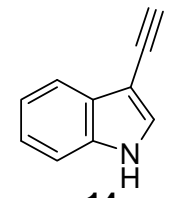

14
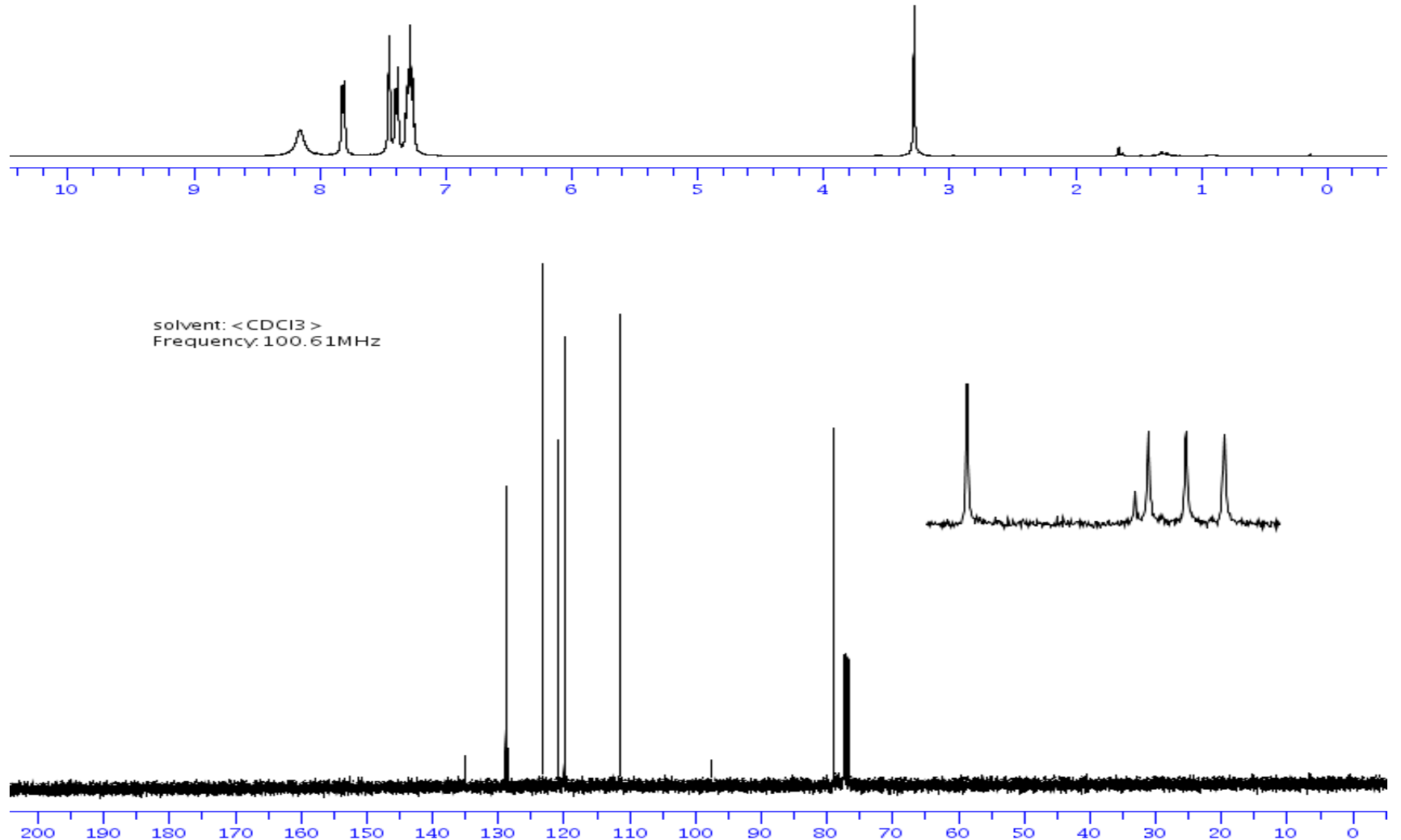

solvent: $<\mathrm{CDCI} 3\rangle$ Frequency. $100.61 \mathrm{MHz}$
DEPT 135

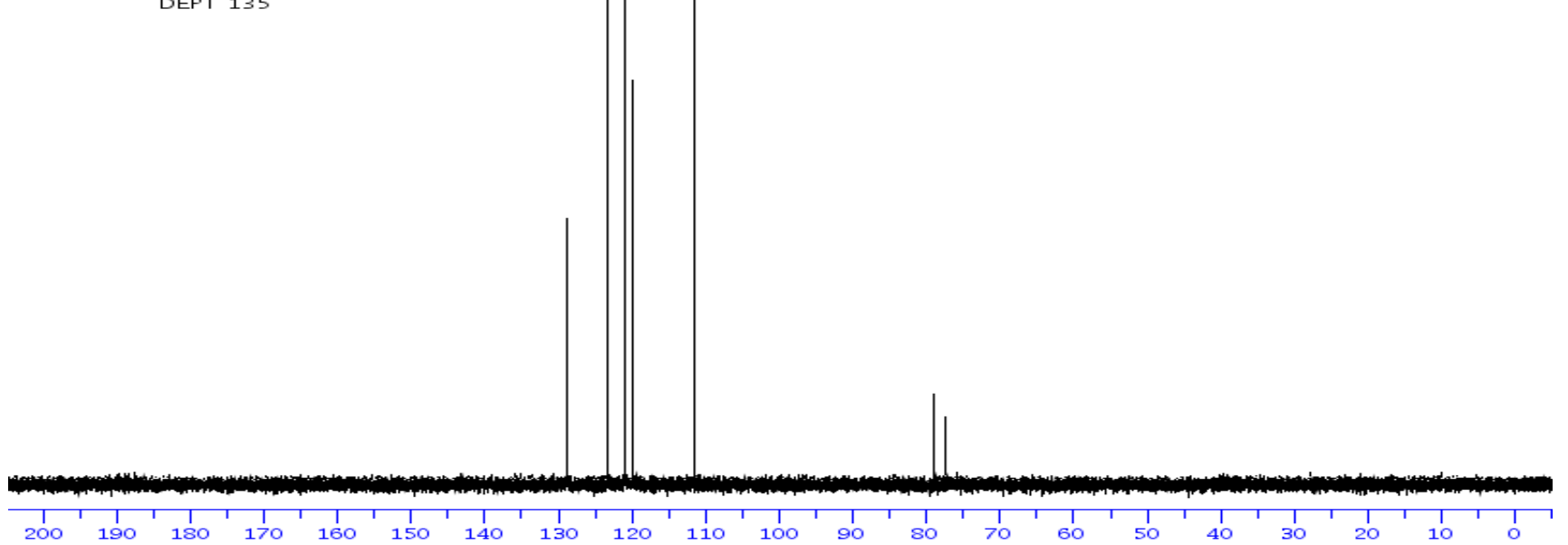


solvent: $<\mathrm{CDCI} 3>$

Frequency $400.13 \mathrm{MHz}$

$\overbrace{N}^{D}$
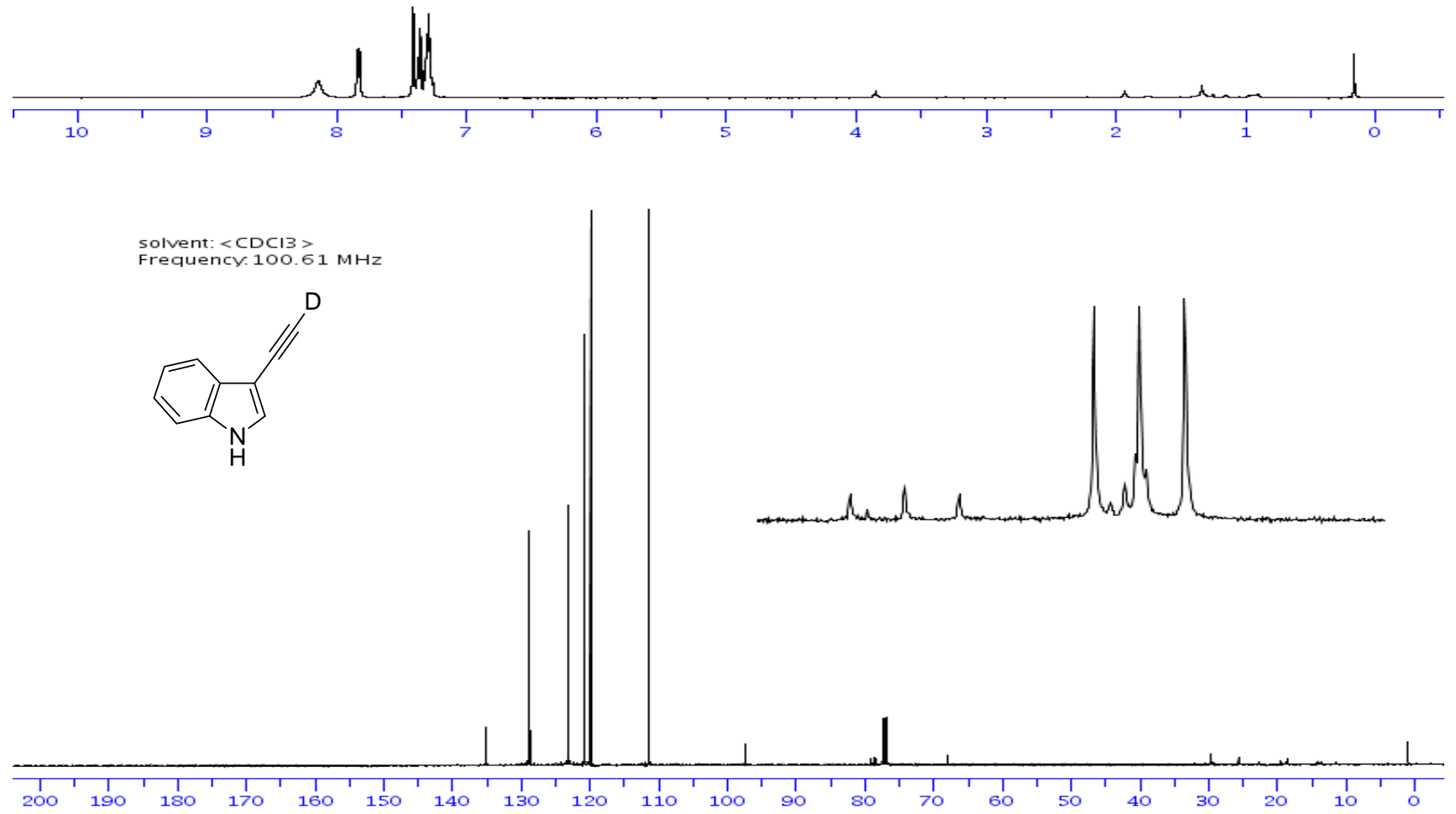
solvent: $<\mathrm{CDCl} 3\rangle$

Frequency 400 .13 $\mathrm{MHz}$

$\underbrace{}_{23} 13$
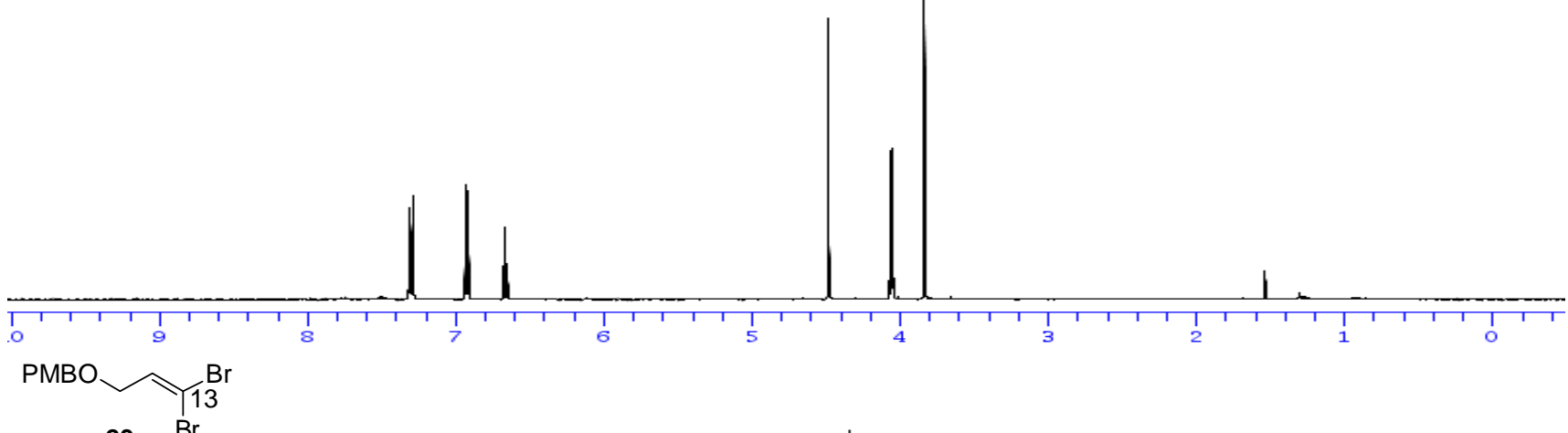

23

Br

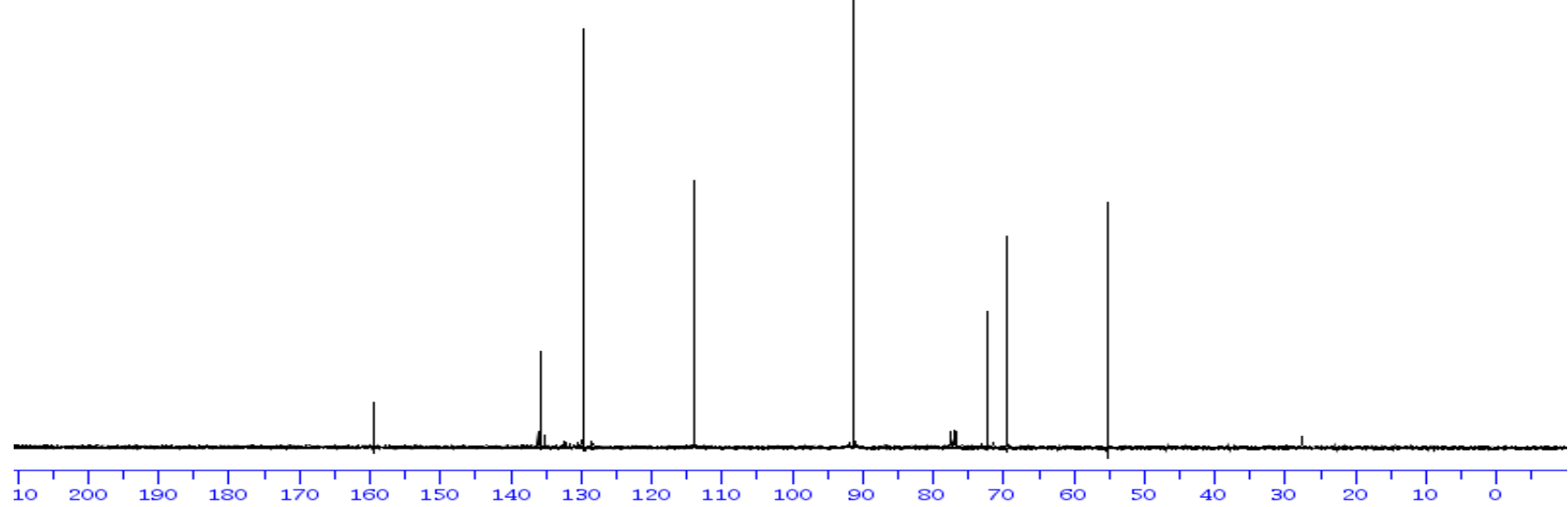

Solvent: $<C D C I 3>$
Frequency. $100.612769 \mathrm{MHz}$

$\mathrm{PMBO}$<smiles>CCC=C(Br)Br</smiles>

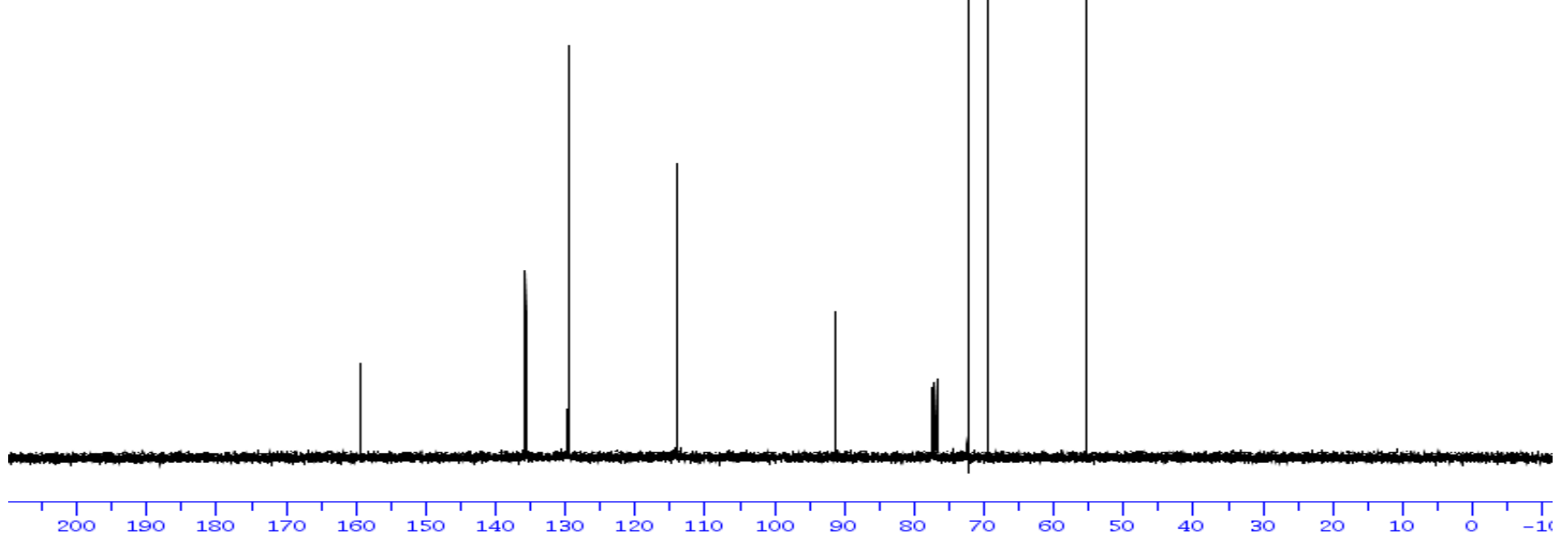


solvent: $<\mathrm{CDCI} 3\rangle$

Frequency.400.13 $\mathrm{MHz}$

PMBO $/ 13^{\mathrm{SiiPr}_{3}}$

24
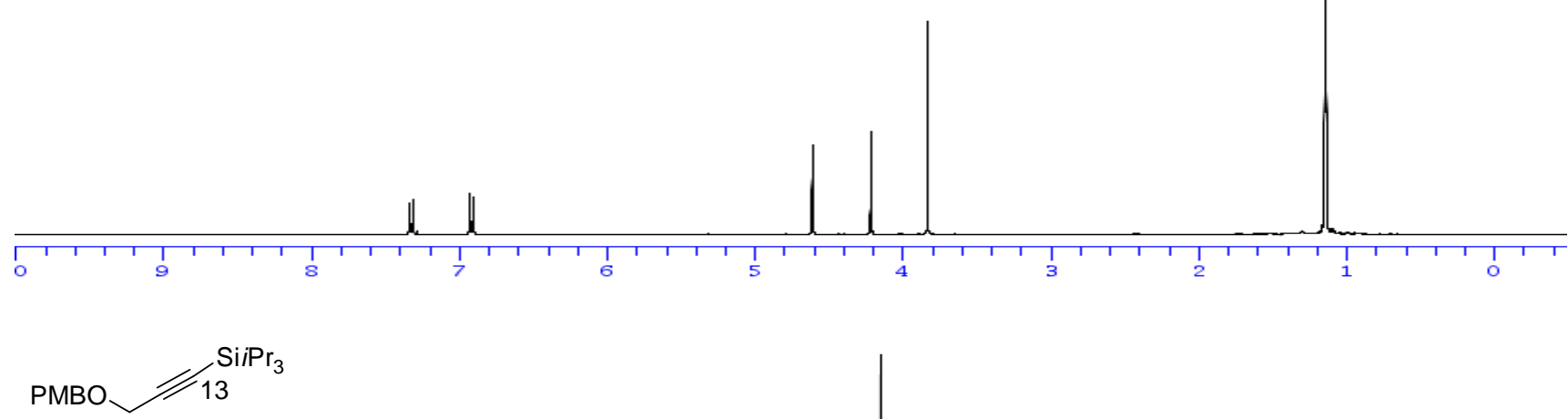

24

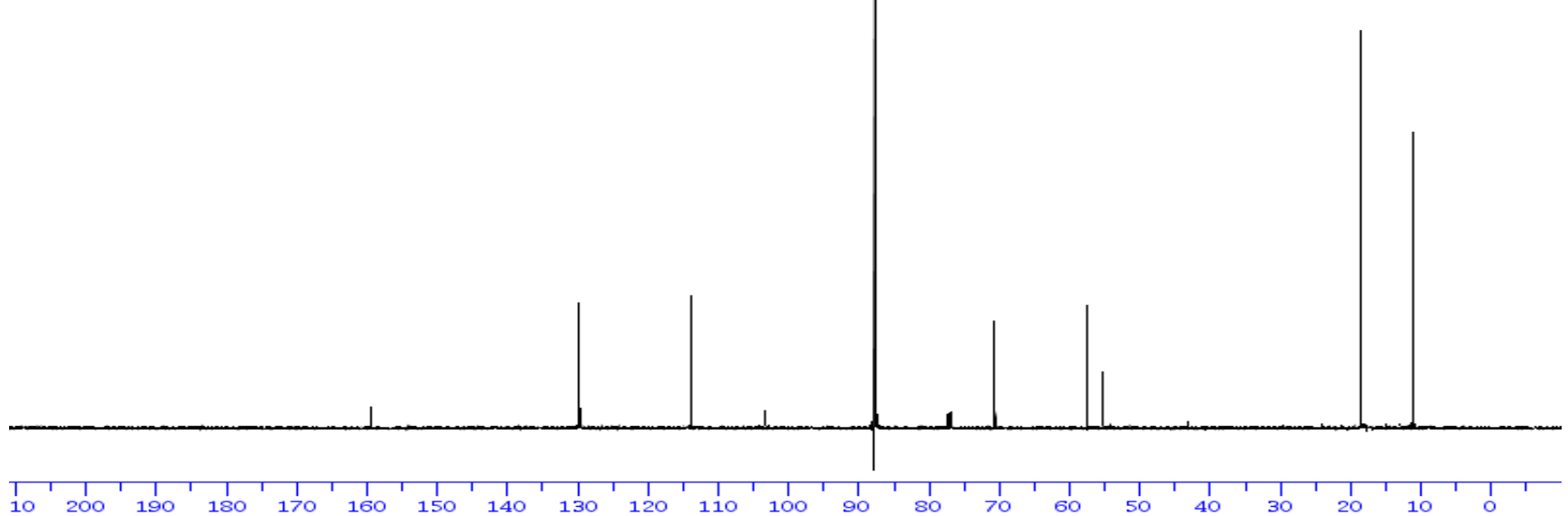

solvent: $\langle\mathrm{CDCl} 3\rangle$

Frequency. 100.612769M Hz

$\mathrm{PMBO} \sim^{\mathrm{SiiPr}_{3}}$

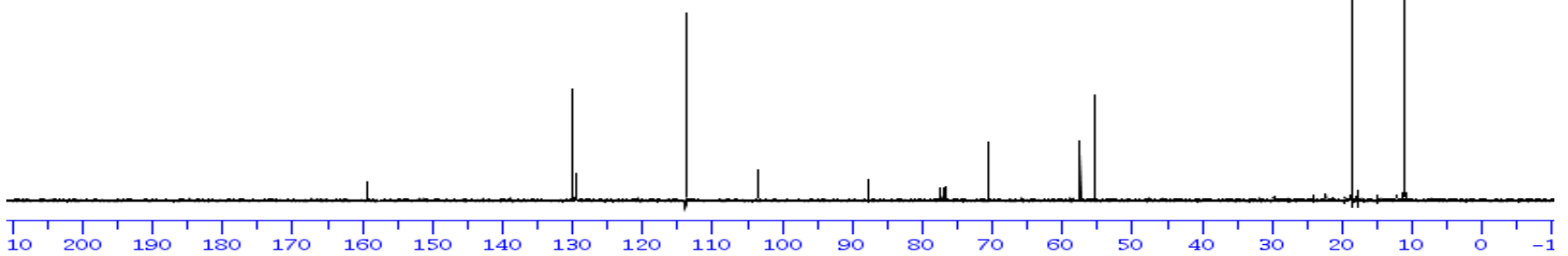


solvent: $<\mathrm{CDCI} 3$

Frequency.400.13 $\mathrm{MHz}$

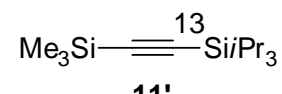

$11^{\prime}$

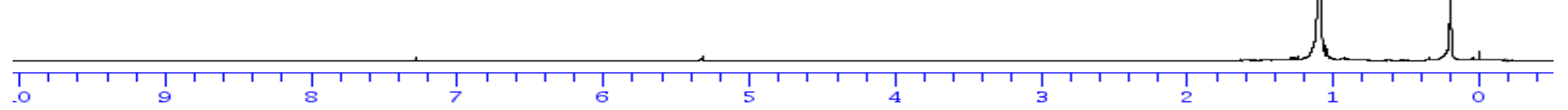

solvent: $<\mathrm{CDCI} 3>$

Frequency. $100.612769 \mathrm{MHz}$

$$
\mathrm{Me}_{3} \mathrm{Si}={ }^{13} \mathrm{SiiPr}_{3}
$$

$11^{\prime}$

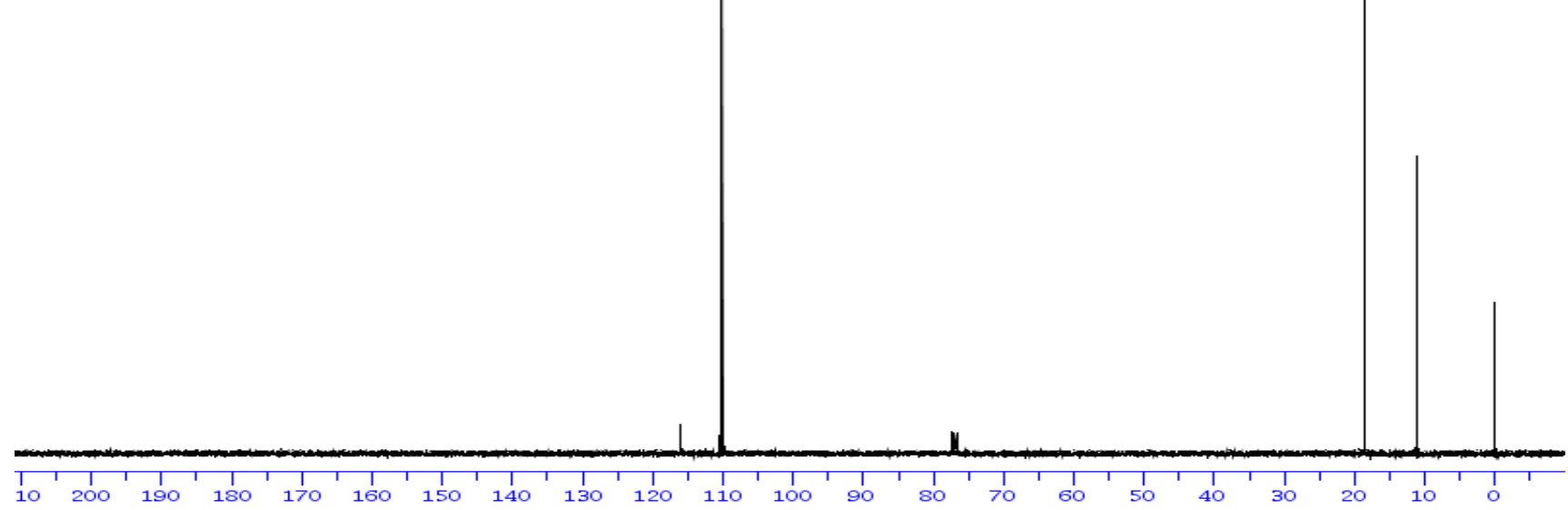

solvent: $<\mathrm{CDCl} 3\rangle$

Frequency. $100.61 \mathrm{MHz}$

$\mathrm{Me}_{3} \mathrm{Si}=\mathrm{SiiPr}_{3}$

11

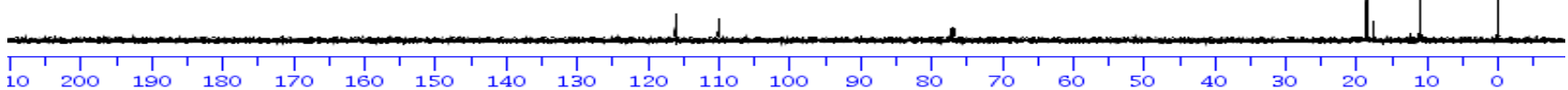


Solvent: $<\mathrm{CDCI} 3>$
Frequency. $400.13 \mathrm{MHz}$
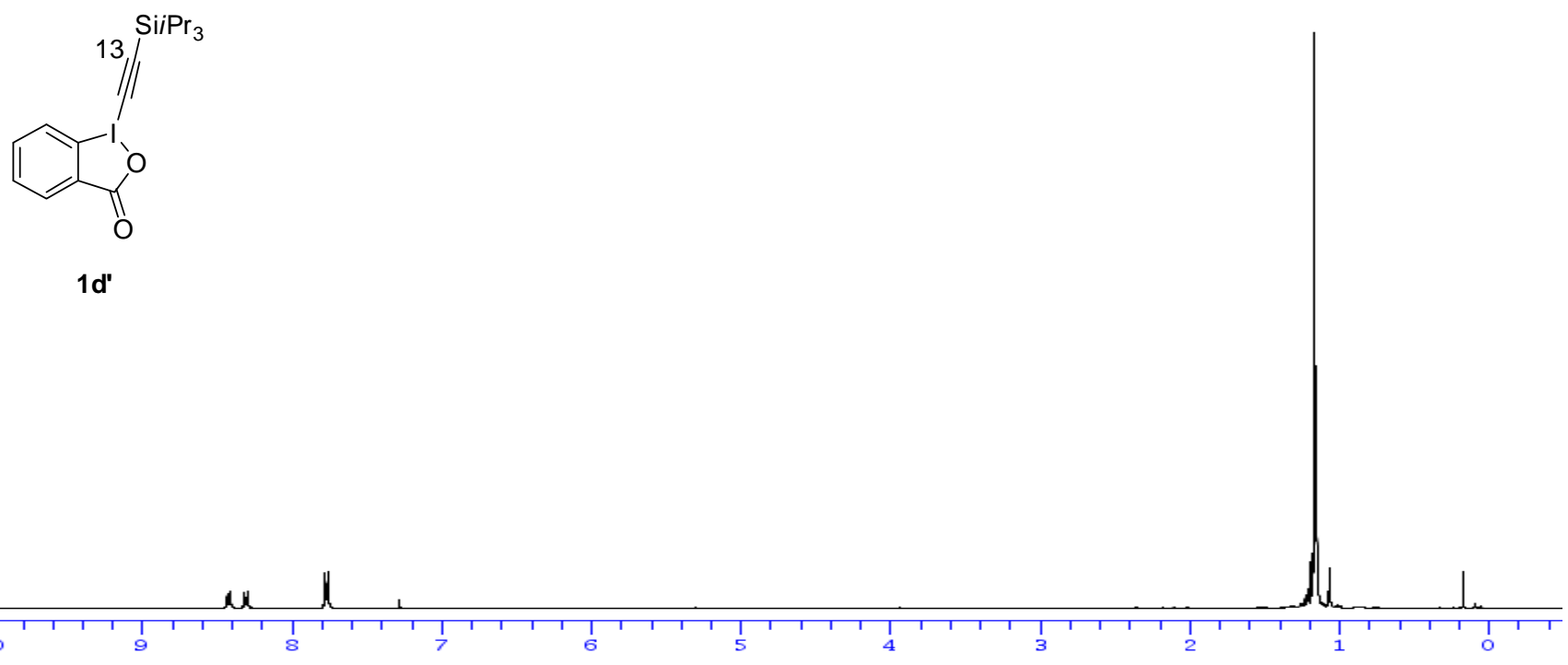

solvent: $<\mathrm{CDCI} 3>$
Frequency. $100.612769 \mathrm{MHz}$

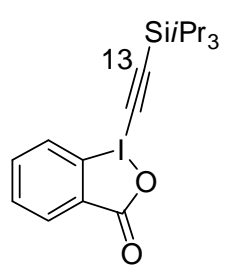

$1 d^{\prime}$

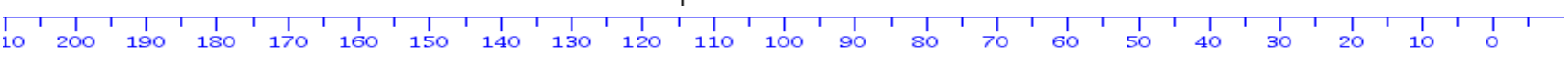
solvent: $<\mathrm{CDCI} 3>$ quincy. $100.61 \mathrm{MHz}$

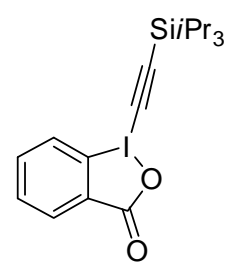

Id

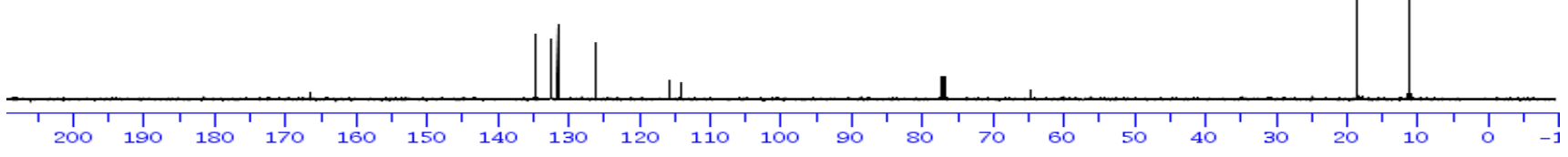

S58 

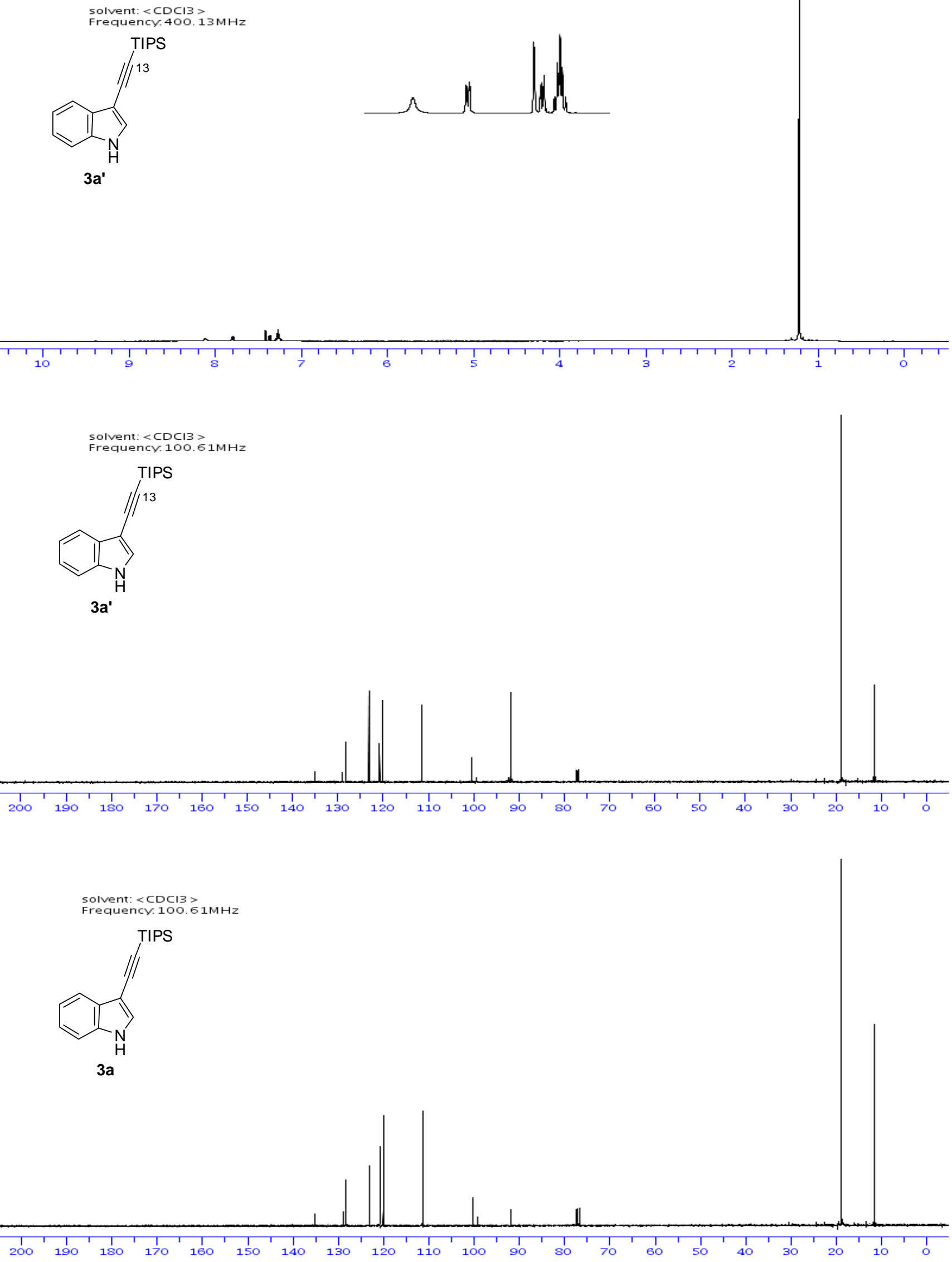

S59 
solvent: $\langle\mathrm{CDCl} 3\rangle$

Frequency. 400.13 $\mathrm{MHz}$<smiles>C#Cc1c[nH]c2ccccc12</smiles>

$14^{\prime}$

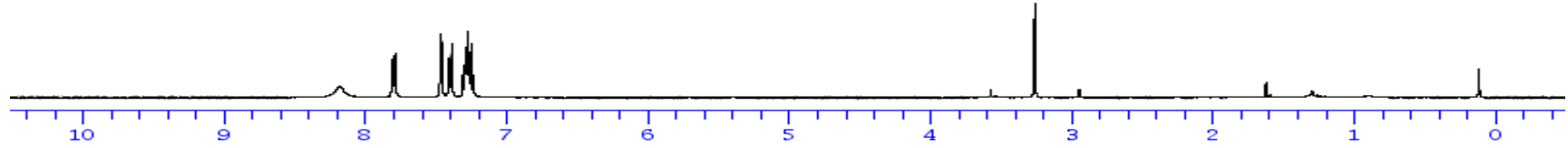

Solvent: $\angle C D C I 3>$
Frequency. $100.61 \mathrm{MHz}$
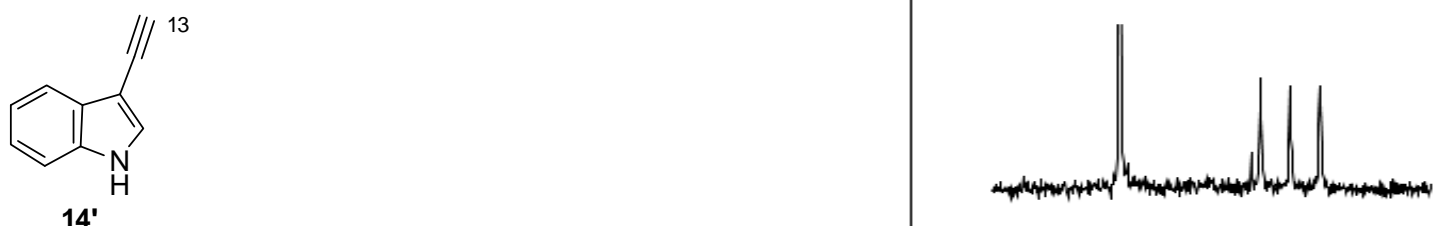

14
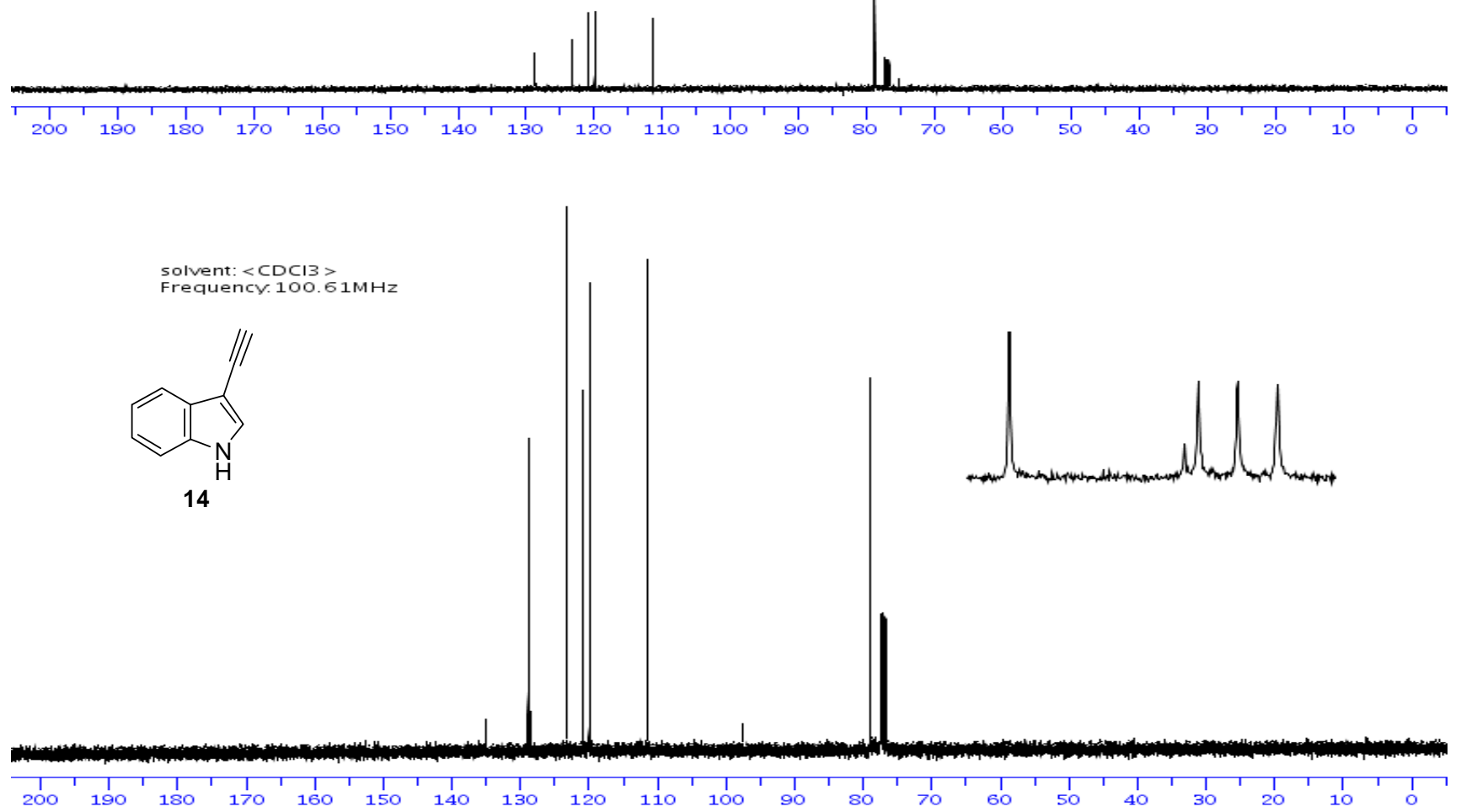